\title{
Risk Factors for Death or Heart Transplant for Patients with Right Ventricular Dysfunction after the Norwood Procedure: A Secondary Analysis of the Single Ventricle Reconstruction Trial
}

by

Emilie Melissa Jean-St-Michel, MD

A thesis submitted in conformity with the requirements

for the degree of Master of Science

Clinical Epidemiology and Health Care Research

University of Toronto

(C) Copyright by Emilie Jean-St-Michel 2017 


\title{
Risk Factors for Death or Heart Transplant for Patients with Right Ventricular Dysfunction after the Norwood Procedure: A Secondary Analysis of the Single Ventricle Reconstruction Trial
}

\author{
Emilie Melissa Jean-St-Michel \\ Master of Science \\ Clinical Epidemiology and Health Care Research \\ University of Toronto
}

2017

\section{Abstract}

We investigated patient and procedural factors associated with failure of staged palliation after the Norwood procedure in patients with right ventricular dysfunction (RVDfx).

Patients from the Pediatric Heart Network Single Ventricle Reconstruction (SVR) Trial dataset were selected if RVDfx was present, defined as an ejection fraction of less than $44 \%$ and a fractional area of change of less than $35 \%$ on the post-Norwood echocardiogram. Transplantfree survival after the Norwood procedure was analyzed using multiphase parametric hazard analysis and factors associated with death or transplant were identified.

In the SVR cohort, 123 (34\%) infants had RVDfx. The six-month transplant-free survival was $87 \%(70 \% \mathrm{Cl} 82-91 \%)$. The independent factors associated with increased risk of death or transplant were BT shunt, increased RV size, infectious disease complications and low surgeon volume.

Patient factors can help identify which patient with RVDfx is at higher risk of death and help decision making around early transplant listing. 


\section{Acknowledgments}

Preparing my master thesis has been a very fulfilling experience allowing me to learn a new statistical technique and working on my writing skills. I am very thankful for the opportunity to grow further as a researcher and I am convinced that the skills that I have learned in the process will serve as the foundation for future research. I would like to take the opportunity to thank my supervisor, Brian McCrindle, who patiently answered all my many questions and allowed me to be successful in this endeavor. I also want to thank all the thesis committee members, Brian McCrindle, Jonathon Maguire and John Coles, who helped me select a project that was feasible within the master thesis timeline and for sharing their knowledge and wisdom throughout the process. I would like to acknowledge James Meza for all the hours he spent with me while I was learning parametric hazard modeling. I also want to mention Anne Dipchand for graciously offering to cover my clinical service while I attended all the master classes and study groups. Finally, I want to thank my husband, Renaud Dutrisac, for his support while I completed this degree and settled into my first year as a staff cardiologist at the Hospital for Sick Children. I thank him for his encouragements as I reach my goals and dreams. Finally, many thanks to my little guy, Eliott, who did not help at all for this thesis project but certainly makes me smile and laugh on a daily basis. 


\section{Contributions}

The study presented in this manuscript was completed with the contribution of a number of coauthors listed below with their name, institutional affiliations and contributions. In addition, this study would not have been possible without access to the dataset of the Single Ventricle Reconstruction trial from the Pediatric Heart Network.

Brian W. McCrindle, MD (The Hospital for Sick Children, Toronto, Ontario, Division of Pediatric Cardiology): Guidance on study design and statistical analysis, review of manuscript.

John Coles, MD (The Hospital for Sick Children, Toronto, Ontario, Department of Cardiovascular Surgery): Guidance on study design and statistical analysis, review of manuscript.

Jonathon Maguire, MD MSc (St. Michael's Hospital, Toronto, Ontario, University of Toronto Department of Pediatrics): Guidance on study design and statistical analysis, review of manuscript.

James M. Meza, MD (The Hospital for Sick Children, Toronto, Ontario, CHSS Data Center): Guidance on statistical analysis. 


\section{Table of Contents}

Heading Page

Title Page $\quad$ i

$\begin{array}{ll}\text { Abstract } & \text { ii }\end{array}$

$\begin{array}{ll}\text { Acknowledgements } & \text { iii }\end{array}$

Contributions $\quad$ iv

Table of Contents $\quad$ V

List of Tables $\quad$ xii

List of Figures $\quad$ xiii

1. Background 1

1.1 Pediatric Heart Network Single Ventricle Reconstruction trial 1

1.2 Hypoplastic left heart syndrome 2

1.2.1 Definition and epidemiology 2

1.2.2 Presentation 4

1.2.3 History of surgical repair in HLHS 4

1.2.4 Current surgical repair 5

1.2.4.1 First stage palliation surgery $\quad 5$

1.2.4.2 The goals and risks of the stage I surgery 8 
1.2.6.3 Risk factors for mortality in the Single Ventricle Reconstruction trial 
1.2.7.2.3 Definition of right ventricular dysfunction by two-dimensional echocardiography

1.2.7.3 Incidence of right ventricular dysfunction in HLHS

1.2.7.5 Association of ventricular dysfunction with mortality in the Single Ventricle Reconstruction trial

1.2.7.6 Association between mortality and ventricular dysfunction in other analyses

1.2.7.6 Ventricular dysfunction is not always a permanent diagnosis

1 2.7.7 Ventricular dysfunction is a common reason for heart transplant listing 
3.2.2 Protocolized echocardiograms in the Single Ventricle

Reconstruction trial $\quad 30$

3.2.2 Right ventricular function measurements 31

3.3 Inclusion and exclusion criteria 31

3.4 Human Research consideration 32

3.5 Outcome measures 32

3.6 Data collection 32

3.7 Sample size 33

3.8 Statistical Methodology 33

3.8.1 Descriptive statistics 33

3.8.2 Data imputation 34

3.8.3 Time to event analysis $\quad 34$

$\begin{array}{ll}3.8 .4 \text { Censoring } & 37\end{array}$

3.8.5 Hazard modeling $\quad 37$

3.8.5.1 Model the hazard phases $\quad 37$

3.8.5.2 Goodness of fit of the parametric model $\quad 40$

3.8.6 Identification of associated factors $\quad 40$

3.8.7 Nature and influence of risk factors $\quad 41$

3.8.8 Best and Worst-case scenario predictions 42

3.8.9 Assumptions and model verification $\quad 42$ 
4.1 Characteristics of the cohort $\quad 43$

4.1.1 Identification of the study cohort 43

4.1.2 Characteristics of the right ventricular dysfunction cohort 44

4.1.3 Baseline echocardiogram results for the right ventricular dysfunction cohort 45

4.1.4 Post-Norwood echocardiogram results for the right ventricular $\begin{array}{ll}\text { dysfunction cohort } & 46\end{array}$

$\begin{array}{lr}\text { 4.1.5 Descriptives statistics } & 50\end{array}$

4.2 Comparison of the ventricular dysfunction cohort to the normal right $\begin{array}{ll}\text { ventricular function cohort } & 50\end{array}$

4.2.1 Comparison of the patient characteristics $\quad 50$

4.2.2 Comparison of the baseline echocardiogram measurements 52

4.2.3 Comparison of the post-Norwood echocardiogram measurements 53

4.3 Association of shunt type with ventricular function 53

4.4 Risk-unadjusted parametric survival analysis for the ventricular dysfunction cohort $\quad 54$

4.4.1 Left censoring $\quad 54$

4.4.2 Hazard modeling $\quad 56$

4.5 Risk factors associated with death or transplant in the ventricular $\begin{array}{ll}\text { dysfunction cohort } & 59\end{array}$

4.5.1 Variable selections $\quad 59$ 
5.1.2 Effect of shunt type on right ventricular function in the SVR trial 81

5.2.2 The relation between ventricular dysfunction and ventricular dilatation 
5.6 Future direction

5.7 Study limitation

5.7.1 Selection bias

5.7.2 Information bias

97

5.7.3 Confounding

98

5.7.4 Left censoring

98

5.7.5 Power

98

5.8 Clinical implications

99

5.9 Conclusion

99

Tables

100

References

115

Copyright acknowledgements

125 


\section{List of Tables}

Chapter 1: (none)

Chapter 2: (none)

Chapter 3:

1. Predictor variables included in the analysis

Chapter 4:

2. Demographic and Norwood procedure hospitalization information for the ventricular dysfunction and the normal function cohorts

3. Comparison of baseline echocardiographic measurements between the ventricular dysfunction and the normal function cohorts

4. Comparison of post-Norwood echocardiographic measurements between the ventricular dysfunction and the normal function cohorts

5. Independent risk factors for death or heart transplant without achieving the stage II procedure

Chapter 5: (none) 


\section{List of Figures}

\section{Chapter 1:}

1. Hypoplastic left heart syndrome diagram

2. Stage surgical palliation for hypoplastic left heart syndrome

3. Modified Blalock-Thomas-Taussig shunt and right ventricle pulmonary artery conduit

4. The balancing act of the systemic and pulmonary vascular resistances in infants post-Norwood procedure

5. The Hybrid approach

6. Example of measurement of right ventricular area in transverse apical fourchamber view obtained by echocardiography necessary for calculation of RVFAC and RVEF

7. Right ventricular cavity length measured on the subcostal sagittal view from right ventricular apex to pulmonary valve

Chapter 2: (none)

Chapter 3:

8. Left censoring

9. Time to death, transplant or stage II

10. The functions used to describe the time related event with parametric modeling

\section{Chapter 4:}

11. Study subjects flow chart

12. Changes in RVEF measurements between the baseline and the post-Norwood echocardiogram

13. Changes in RVFAC measurements between the baseline and the postNorwood echocardiogram

14. Changes in tricuspid valve regurgitation severity between the baseline and the post-Norwood echocardiogram 
15. Non-parametric Kaplan-Meier curves for freedom from death or transplant stratified by cohort

16. Distribution of time in days from the Norwood procedure to the post-Norwood echocardiogram

17. Goodness of fit of the constant phase parametric model

18. Goodness of fit of the late phase parametric model

19. Transplant-free survival over time in months for a hypothetical average patient with all the risk factor values set as means

20. Transplant-free survival over time in months for a hypothetical average patient with all risk factor values set at means stratified by shunt type

21. Transplant-free survival over time in months for a hypothetical average patient with all risk factor values set at means stratified by the presence of infectious disease complication post-Norwood procedure.

22. Transplant-free survival overtime in months for a hypothetical average patient with all the risk factor values set as means stratified by surgeon volume with less or equal to 5 Norwood procedures per year compared to more than five

23. Nomogram representing the influence of post-Norwood right ventricular enddiastolic dimension on transplant-free survival at six months post-Norwood procedure for the hypothetical average patient stratified by shunt type

24. Average parametric transplant-free survival for the ventricular dysfunction cohort patients

25. Average parametric transplant-free survival for the ventricular dysfunction cohort patients stratified by shunt type

26. Average parametric transplant-free survival for the ventricular dysfunction cohort patients stratified by the presence or absence of infectious disease complications post-Norwood procedure

27. Average parametric transplant-free survival for the ventricular dysfunction cohort patients stratified by surgeon volume with less or equal to 5 Norwood procedures per year compare to more than five 
28. Nomogram representing the influence of post-Norwood right ventricular enddiastolic dimension on transplant-free survival at six months post-Norwood procedure for ventricular dysfunction cohort patients stratified by shunt type

29. Best and worst case scenario survival predictions

30. Best and worst case scenario survival predictions stratified by shunt type

31. Nomogram representing the influence of post-Norwood right ventricle enddiastolic dimension on transplant-free survival at six months post-Norwood procedure on the best and worst case scenarios stratified by shunt type

32. Deviance residual plotted by unique identifiers

33. Goodness of fit of the final multivariable parametric model

\section{Chapter 5:}

33. The BT shunt anatomy

34. Solution of a mathematical model for BT shunt, RVPA conduit and hybrid approach

blood flow

35. Diagram of the BT shunt and RVPA conduit surgeries

36. The Laplace law 


\section{Chapter 1 \\ Background}

\subsection{Pediatric Heart Network Single Ventricle Reconstruction trial}

The National Heart, Lung, and Blood Institute (NHLBI) established the Pediatric Heart Network (PHN) in 2001 to respond to the need for evidence-based approaches in pediatric cardiology (Mahony et al. 2006). The PHN consists of a data-coordinating center and a consortium of clinical sites across the United States and Canada that conducts research studies in children with congenital or acquired heart disease. The primary objective is to improve outcomes and quality of life in children with heart disease, and represents a cooperative agreement between participating clinical centers, a data-coordinating center, an independent protocol chair, and the NHLBI staff. The PHN has led multiple clinical trials in the field of paediatric cardiology and continue to do so. One of the most important trials performed by the PHN was the Single Ventricle Reconstruction (SVR) trial. This trial represents the first multicenter randomized clinical trial evaluating the surgical outcomes of infants born with Hypoplastic left heart syndrome (HLHS), a severe congenital heart disease associated with high mortality. HLHS and its associated surgeries, treatments and outcomes will be reviewed in more detail in the next section (Section 1.2).

The PHN SVR trial compared outcomes in 555 infants undergoing a Norwood procedure randomized to either a Blalock-Taussig shunt (BT shunt) or a Right ventricular to pulmonary artery conduit (RVPA conduit) at 14 North American centers (Ohye et al. 2008). This was a non-blinded randomized trial published in 2010 in the New-England Journal of medicine (Ohye et al. 2010). All the data collected for this trial has now been completely de-identified and the dataset is now publicly accessible for secondary analyses. The PHN SVR trial has already led to 
multiple publications. The main aim of this randomized trial was to compare 12-month transplant-free survival by shunt types (BT shunt vs. RVPA conduit) for the Norwood procedure. The main result from this trial was an improved transplant-free survival rate at 12 months postNorwood procedure in subjects who received a RVPA conduit versus BT shunt (74 \% vs. 64 \%, $\mathrm{p}=0.01$ ). Multiple secondary analyses have been published using this dataset. Examples include evaluation of the interstage mortality (Ghanayem et al. 2012), risk factors for death or transplant in the intermediate term (Tweddell et al. 2012) and 3 years term (Newburger et al. 1993), as well as shunt type effect on the cardiac function, ventricular size (Frommelt et al. 2012), and ventricular deformation (Hill et al. 2015). However, the group of patients with ventricular dysfunction has not been evaluated separately. This dataset, in addition to providing access to the largest cohort of infants with HLHS post-Norwood procedure, also affords the opportunity for serial protocolized quantitative assessment of right ventricular function (Frommelt et al. 2012). All the SVR trial echocardiograms were done at protocolized times and were all read by the same expert at a core lab (Ohye et al. 2008), with potentially better accuracy for the ventricular dysfunction cohort selection.

\subsection{Hypoplastic left heart syndrome}

\subsubsection{Definition and epidemiology}

Hypoplastic left heart syndrome (HLHS) is defined by the presence at birth of underdeveloped left-sided cardiac structures such as a small left ventricle, a small mitral valve, or a small aortic valve (Noonan \& Nadas 1958) (Figure 1). Within this syndrome, the anomalies of the left-sided cardiac structures have a varying spectrum of severity. For example, the mitral valve can be either small or atretic (when the valve does not open), blocking blood from entering the left ventricle. Despite the variation in severity, this syndrome, by definition, always leads to the inability of the left cardiac structures to sustain adequate systemic cardiac output. In other words, these children are born with only one working ventricle, their morphological right ventricle. HLHS does not represent a uniform group of patients, since multiple cardiac malformations can cause obstructions to and obstructions from the left ventricle. There is the classic HLHS anatomy 
(Figure 1) with a small or atretic left-side structure. However, within the same syndrome, we also find patients born with unbalanced atrioventricular septal defect (AVSD) or with a single ventricle and two atrioventricular valves (double inlet ventricle) as well as other variations. Fundamentally, all these malformations lead to the inability of the left side of the heart to fulfill its purpose. HLHS is a rare syndrome with an incidence of approximately two per 10,000 live births. In fact, this syndrome represents about $2 \%$ of congenital heart diseases but does account for the majority of infant cardiac deaths caused by congenital heart diseases.

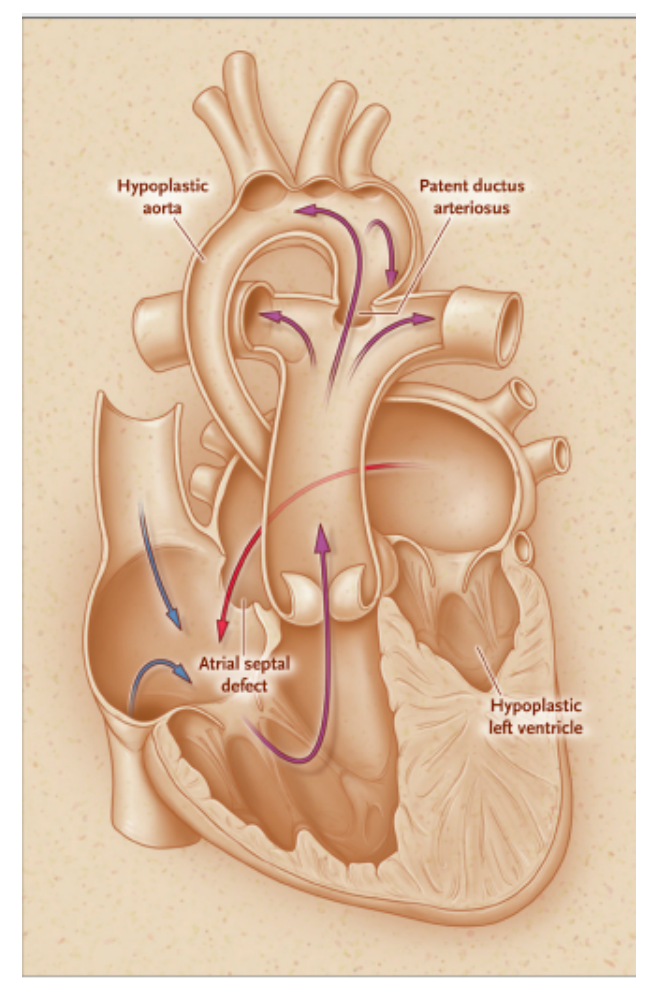

Figure 1. Hypoplastic left heart syndrome diagram. This syndrome is associated with underdevelopment of the left cardiac structures. Therefore, oxygenated blood arriving from the lungs via the pulmonary veins, crosses into the right side of the heart via the atrial communication, and is ejected by the right ventricle. The oxygenated blood then mixes with the deoxygenated blood in the right atrium and get pump into the pulmonary artery. The mixed blood then goes either to the lungs via the pulmonary arteries or to the body via the patent ductus arteriosus (PDA), a remnant of fetal life. Reproduced with permission from (Ohye et al. 2010.), Copyright Massachusetts Medical Society. 


\subsubsection{Presentation}

Currently, most patients are diagnosed with this disease in fetal life. This congenital anomaly is usually diagnosed in utero on screening obstetrics ultrasounds since HLHS can be characterized by an abnormal four-chamber view, which is a standard part of a screening obstetric ultrasound. Following this, the mother is usually referred to a pediatric cardiology service for a complete fetal cardiac echocardiography in order to confirm and specify the diagnosis. Following confirmation of the diagnosis, the information relating to the outcomes and therapeutic options is discussed with the family. If continuation of pregnancy is desired, a plan of care is put into place for immediate and specialized medical attention after birth. Despite this, a quarter of infants will not be diagnosed in utero (Atz et al. 2010) for a variety of reasons, such as the presence of poor ultrasound windows, poor imaging technique, or lack of prenatal care. One quarter of these patients will develop symptoms within the first 24 hours of life and almost all of them will develop symptoms within the first week after birth. The symptoms usually include cyanosis, difficulty breathing, difficulty feeding, and rapid progression to congestive heart failure, shock, and even death.

\subsubsection{History of surgical repair in HLHS}

Without surgical treatment, this severe congenital heart anomaly can be fatal, usually within the first weeks of life. Until the early 1980s, families were offered comfort care as the only therapeutic option. The first surgery for HLHS was attempted in 1961 but the first report of survivors was not until the 1970s (Ohye et al. 2016; Noonan \& Nadas 1958).

This complicated anatomy and the presence of the high pulmonary vascular resistance in newborns led to the development of a staged palliative surgical approach by Norwood et al (Norwood et al. 1981). With this staged surgical approach, patients undergo a minimum of three surgeries, with the last stage being the Fontan procedure, a surgery initially reported as successful in patients born with tricuspid atresia and a hypoplastic right ventricle (patient with only a working left ventricle) (Figure 2). This represents the last surgical step, which ultimately allows complete separation of the oxygenated and deoxygenated blood by diverting the superior 
and inferior vena cava blood directly to the pulmonary arteries without passing through a pumping chamber. This reserved the working single ventricle for systemic perfusion only, allowing children to have normal or near normal oxygen saturation.

\subsubsection{Current surgical repair}

\subsubsection{First stage palliative surgery}

In the current era, these children still need to undergo three surgeries. The initial surgery (stage I) is done a few days after birth and consists of aortic arch reconstruction, transection of the pulmonary arteries, anastomosis of the main pulmonary artery and small ascending aorta by creating a "neo-aorta" in order to supply systemic circulation; opening of the atrial communication; and the addition of a controlled source of blood flow to the transected pulmonary arteries (Figure 2). Two main surgical techniques exist for the addition of a controlled source of pulmonary blood flow: the modified Blalock-Thomas-Taussig shunt, commonly called the modified Blalock-Taussig shunt (BT shunt), and the right ventricular to pulmonary artery (RVPA) conduit, commonly called the Sano shunt (Figure 3). The BT shunt consists of a three to five millimeter artificial tube that is attached between the subclavian artery and the branch pulmonary arteries. The RVPA conduit consists of a similar sized artificial tube attached to the branch pulmonary arteries and also to the right ventricle via a ventriculotomy (an incision in the right ventricular wall). Both surgical procedures use a valveless tube graft. These are very complex cardiac surgeries that require the use of a cardiopulmonary bypass, deep hypothermia, and circulatory arrest with or without regional perfusion of the brain. 


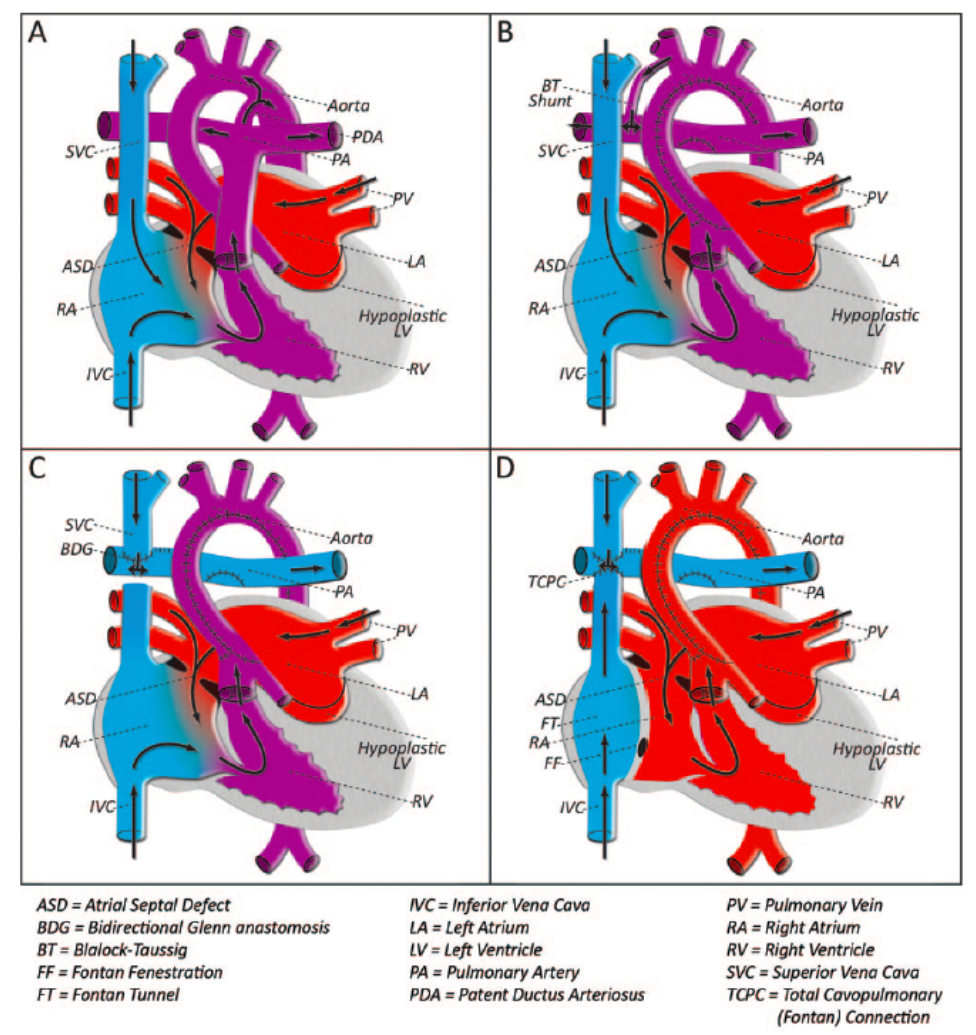

Figure 2. Stage surgical palliation for hypoplastic left heart syndrome. A) Diagram of the presurgical anatomy of patients with classic HLHS. B) Diagram of the Norwood procedure (stage I) consisting of aortic arch reconstruction; with connection of the main pulmonary artery and small ascending aorta; opening of the atrial communication; and the addition of a controlled source of pulmonary blood flow represented on this diagram as a Blalock-Taussig shunt. C) The bidirectional cavo-pulmonary shunt (stage II), which removes the source of pulmonary blood flow created during the first surgery (BT shunt), and attaches the superior vena cava directly to the pulmonary arteries. D) The Fontan procedure (stage III) completely separates the deoxygenated blood from the oxygenated blood by routing the inferior vena cava blood flow to the pulmonary artery. Reprinted by permission from Macmillan Publishers Ltd: Pediatric Research (Albers et al.), copyright (2010). 


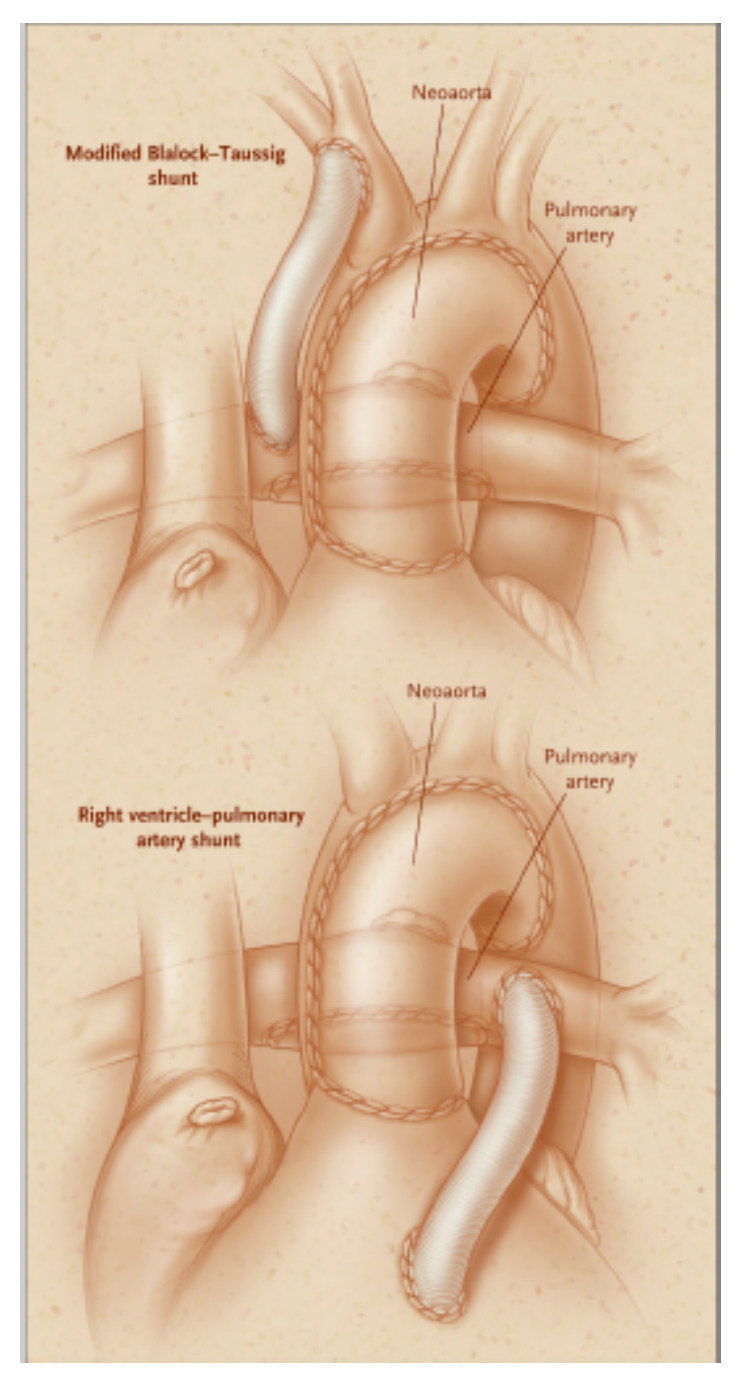

Figure 3. Modified Blalock-Taussig shunt and right ventricle pulmonary artery conduit. The top diagram illustrates the modified BT shunt consisting of an artificial tube anastomosed between the subclavian artery and the branch pulmonary arteries. The lower diagram demonstrates the RVPA conduit consisting of a similar sized artificial tube attached to the branch pulmonary arteries and also to the right ventricle via a ventriculotomy (an incision in the right ventricular wall). Both diagrams also illustrate the patch used to enlarge the aortic arch as well as the patch used to enlarge the branch pulmonary arteries and closed where it was previously attached to the heart. Reproduced with permission from (Ohye et al. 2010.), Copyright Massachusetts Medical Society. 


\subsubsection{The goals and risks of the Stage I surgery}

The ultimate goal of the first stage procedure is to create a balance and stable circulation in order to allow the patient to mature, grow, and lower their pulmonary resistance over the first three to six months of their life. This lung maturation will allow the patient to tolerate the second and third stage surgeries while controlling both the systemic and pulmonary perfusion. However, this procedure leads to a very unique circulation. The pulmonary and systemic circulation in these patients is in parallel (Figure 1 and 2). To illustrate this better, you can imagine a red blood cell that gets pumped out by the right ventricle. This red blood cell can either go through the surgical shunt and end up in the lungs or get pumped into the aorta and end up in the body or brain. In other words, the resistance of both systems, the systemic and pulmonary circulations, will determine whether the red blood cell is more likely to go to the lungs or to the body and brain. This requires the two circulations (pulmonary and systemic) to remain balanced over time. An imbalance in resistance will lead to too much blood going through the lungs and not enough to the body or vice versa, which can have severe consequences (Figure 4). This also results in volume and pressure overloading of the right ventricle since it is exposed to both pulmonary and systemic circulations. These phenomena explain, in part, the poor circulatory reserve of HLHS patients, and the high morbidity and mortality seen between the first and second stage surgeries. 
A)

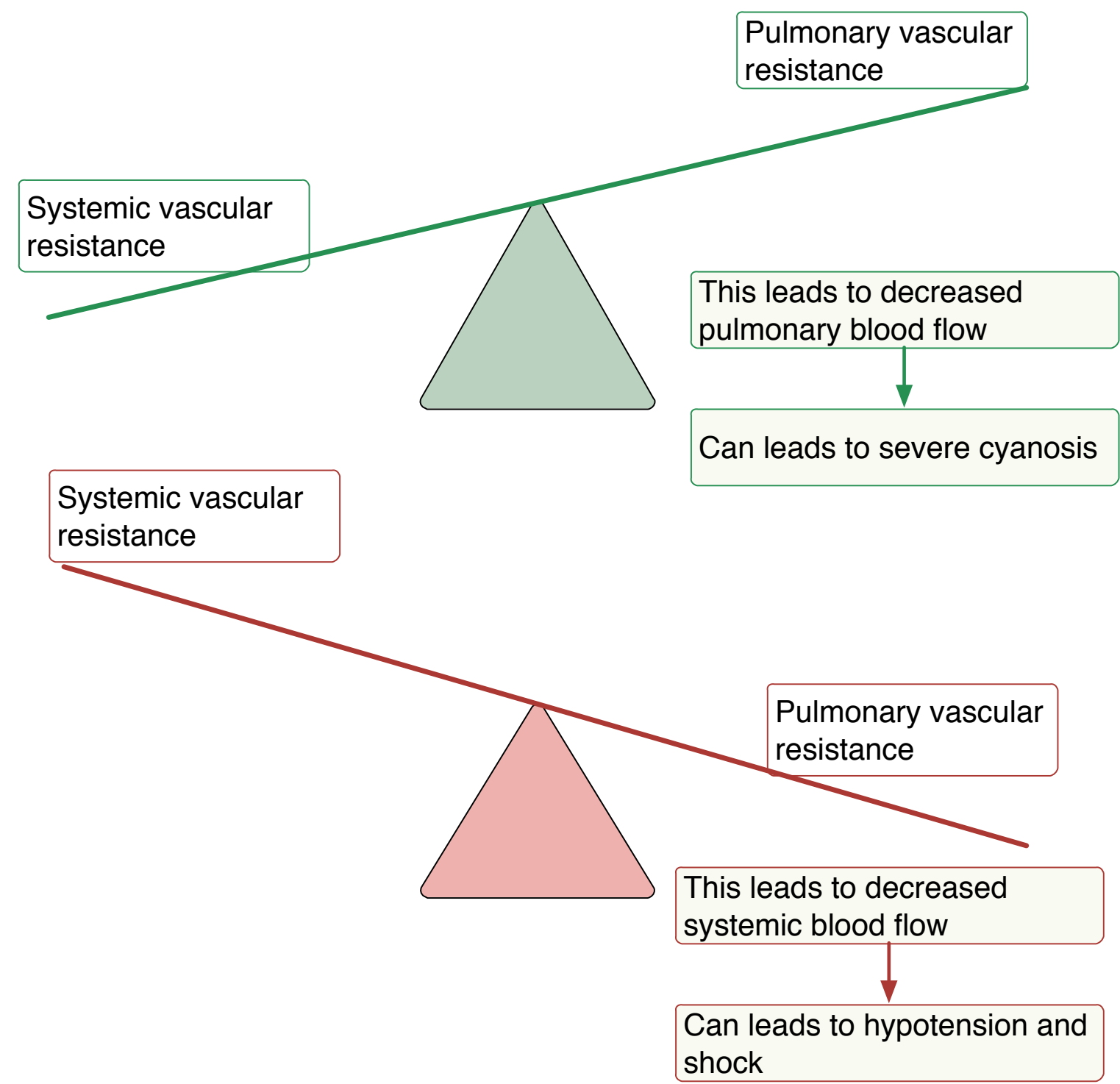

Figure 4. The balancing act of the systemic and pulmonary vascular resistances in infants post-Norwood procedure. Scenario A shows the consequences of higher pulmonary vascular resistance compare to the systemic vascular resistance, leading to low pulmonary blood flow and severe cyanosis. Scenario B shows the consequences of higher systemic vascular resistance leading to low systemic perfusion with associated hypotension and cardiovascular shock. 


\subsubsection{Second stage surgery}

The second palliative surgery (stage II) is the bidirectional cavo-pulmonary shunt (BCPS), also called the bidirectional Glenn anastomosis, which removes the additional source of pulmonary blood flow added at the first surgery (BT shunt or RVPA conduit) (Figure 2 and 3). The surgeon then attaches the superior vena cava directly to the pulmonary arteries; therefore, only the venous blood (deoxygenated blood) travelling from the brain and arms will go through the lungs without passing through a pumping chamber (ventricle) and without mixing with the oxygenated blood which is arriving from the lungs. This surgery requires less extensive surgical reconstruction than during the first stage surgical procedure. This procedure is usually done at four to six months of age.

\subsubsection{The goals and risks of the Stage II surgery}

The stage II surgical procedure allows the patients to have their pulmonary and systemic circulations in series for the first time of their life. As a result, the patients do not depend on a balanced pulmonary and systemic circulation as much as before (Figure 4). This procedure also results in volume unloading of the right ventricle. All these changes explain why the stage II procedure usually represents the end of the higher mortality risk period in this population. However, this surgical stage has particular challenges. Venous blood now flows through the pulmonary system without the benefit of a pump; therefore, high pulmonary vascular resistance

or a variety of lung diseases can lead to high venous pressure, low pulmonary perfusion, and low oxygen saturation. This procedure is delayed to four to six months of age in order to allow for maturation of the lungs, lowering of the pulmonary vascular resistance and weight gain to render the physiology of the superior vena cava anastomosis possible. 


\subsubsection{The Fontan procedure}

Following the second surgery, as the child grows the oxygen saturation usually progressively decreases as the relative portion of cardiac output going through the superior vena cava reduces. The final surgical stage is undertaken when the child is large enough for an adult size connection between the inferior vena cava and the pulmonary artery. This third palliative surgery, the Fontan procedure, consists of an inferior vena cava anastomosis to the pulmonary artery, which allows complete separation of the oxygenated and deoxygenated blood. This surgery is usually done at 18 months to 48 months of life (Figure 2). Two common surgical methods exist for this stage: the lateral tunnel approach and the extracardiac conduit method, both with or without a fenestration. I will not discuss in detail the challenges associated with the Fontan procedure since this is not the subject of this analysis.

\subsubsection{Other therapeutic options}

\subsubsection{The Hybrid approach}

Patients with this congenital cardiac anomaly have other therapeutic options at their disposal other than the staged surgical options described above. There is a combined surgical and interventional catheterization palliation that leads patients through a three-stage approach similar to the staged surgeries described above. The hypothetical advantage of this approach is that it reserves the more complicated surgical aortic arch reconstruction to the second stage procedure when the patient is older, to approximately four to six months of age. This allows avoiding the use of cardiopulmonary bypass, deep hypothermia, and circulatory arrest in the neonatal period, a period of critical brain development. The theory, thought unproven, is that delaying this aspect of the surgical procedure allows for better myocardial and neurological protection in older infant. The initial stage involves a patent ductus arteriosus stent (natural connection between the 
pulmonary artery and aortic arch present in utero and at birth) implanted using cardiac catheterization techniques as well as implantation of a surgical pulmonary artery bands (artificial bands placed around an artery to make it physically smaller) (Figure 5). The second stage then includes a surgical aortic arch reconstruction with an anastomosis of the main pulmonary artery and small ascending aorta to create a "neo-aorta" in order to supply systemic perfusion and coronary arteries perfusion. It also includes enlargement of the atrial communication; and attaching the superior vena cava directly to the pulmonary arteries in order to supply pulmonary perfusion. The third stage procedure is the same Fontan procedure described in the previous section. This intervention based staged approach is called the "hybrid approach", but was not included in the SVR trial (Ohye et al. 2008) from which our analysis is derived.
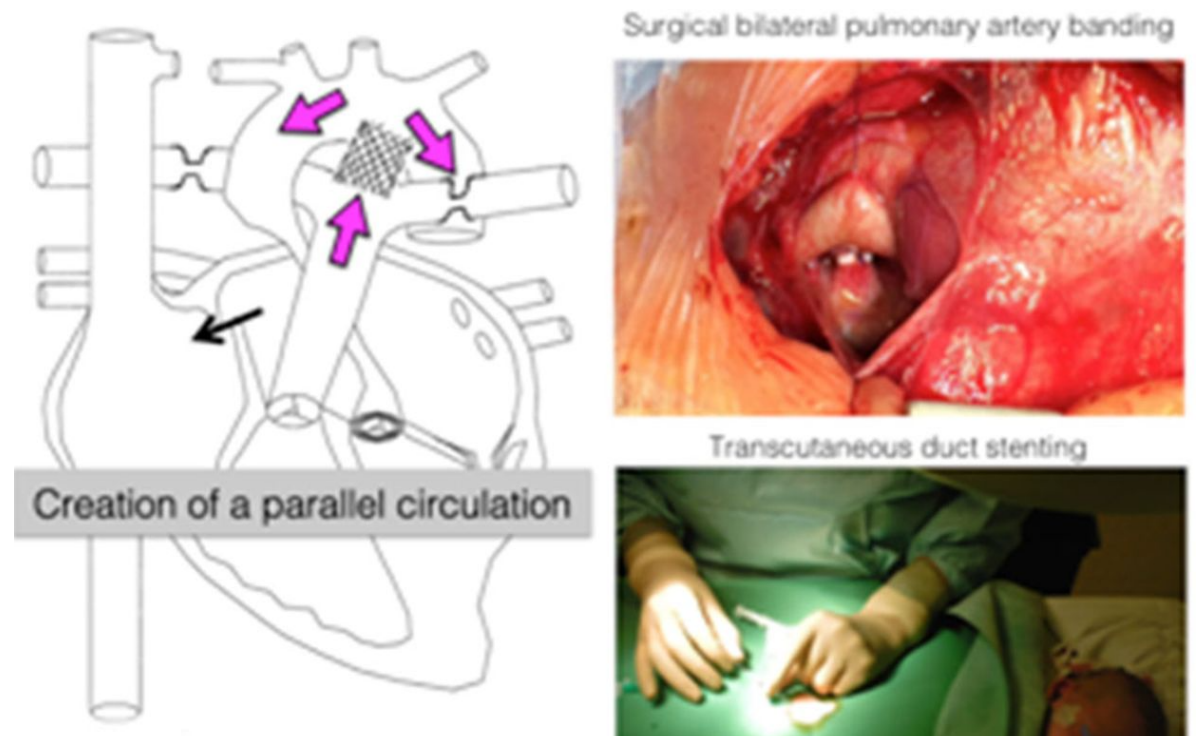

Transcutaneous duct stenting

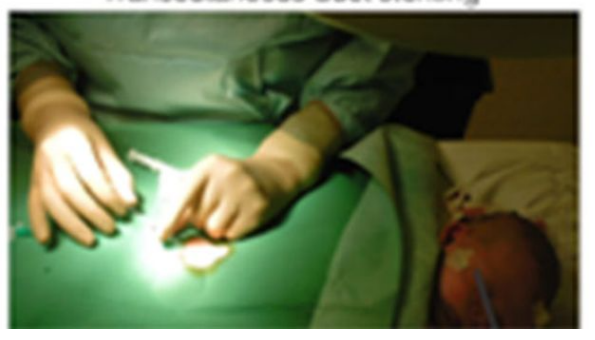

Figure 5. The Hybrid approach. The left drawing demonstrates the ductal stent and the bilateral pulmonary arteries band. The upper right picture shows the open-chest approach for the right pulmonary artery banding. The lower right picture demonstrates the cardiac catherization approach to implant the ductal stent. Reproduce with permission from (Ohye et al. 2016), Copyright Wolters Kluwer Health Inc. 


\subsubsection{Heart transplant}

Families can also choose a primary heart transplant. In the current era of organ shortages, the waiting times to receive a heart are long and this leads to high mortality while waiting for a heart. In addition, HLHS infants with previous surgery are associated with the worst outcomes post-heart transplant (Carlo et al. 2016). In reality, the combined waiting list and transplant mortality far exceed the current interstage mortality (Everitt et al. 2012) and, therefore, is not a favored option.

\subsubsection{Palliative care}

Finally, because of the significant mortality and morbidity that remains associated with this HLHS, palliative care and comfort care remain a therapeutic option for those families, as is elective termination of pregnancy.

\subsubsection{Short-term outcomes and risk factors}

\subsubsection{Definition of mortality}

Even with the surgical and medical improvements of the last two decades, this congenital heart defect continues to be associated with a high mortality rate and morbidity. Compared to the 35 most common structural birth defects, the Norwood procedure was associated with the highest cost and the third longest length of hospital stay using resource utilization data from the United States (Center for Disease Control and Prevention (CDC) 2007). Mortality, as reported in the literature, can be defined using different time intervals, which affects the results of the different analysis. For example, mortality can be defined as preoperative death, thirty-day post-operative mortality, hospital mortality, interstage mortality, mortality to stage II and long-term mortality. Thirty-day post-operative mortality and the hospital mortality evaluate a similar time period. The 
thirty-day time interval uses a predetermined end of observation period, which is 30 days postNorwood surgery. Whereas the hospital mortality time of observation ends when the patient is discharged from hospital. This will lead to a higher variation in length of observation period between patients since some patients will be admitted for one week post stage I surgery and other patient might not be discharge until their stage II procedure at 4 to 6 months of age. This will also vary in between centers since criteria for discharge home will vary in-between hospitals and some centers do not discharge home patients post-Norwood procedure until they have completed the stage II procedure. For this reason, most investigators evaluate the thirty-day mortality and not the hospital mortality. Long-term mortality will be defined in different ways and is usually well defined within the individual studies. Most often, the period of observation is chosen because of a specific question or because of the length of observation of the cohort available to the investigators. Finally, mortality is often analyzed as a composite end-point combining death with heart transplant since heart transplant in a rare event. This leads to the assessment of transplant-free survival, also censoring for patients who received a heart transplant.

\subsubsection{Mortality in the Single Ventricle Reconstruction trial}

The period of time between stage I and stage II palliative surgery is the highest mortality risk period (Ghanayem et al. 2012a) because of the physiology associated with the first stage surgery. Patients with HLHS lack circulatory reserves, especially before the stage II procedure. In addition to having only one functioning volume and pressure loaded ventricle, they have a parallel circulation (Figure 4). The SVR trial enrolled 555 infants with HLHS and a single right ventricle and randomized them between BT shunt and RVPA conduit (Ohye et al. 2010). For the first 12 months after randomization, there were 164 (30\%) patients that died and of those, 88 (54 $\%$ ) occurred between randomization and Norwood procedure hospital discharge. There were also $54(33 \%)$ deaths that occurred from the Norwood procedure hospital discharge to stage II hospital admission and 16 (10) patients died during the stage II hospitalization. Finally, six (4 \%) patients died from the stage II hospital discharge and the 12-month follow-up. Evaluating the composite outcome of death and heart transplant, 144 patients ( $26 \%$ of the 555 enrolled infants) died or received a heart transplant between the randomization and the stage II surgery (Ohye et al. 2012). Out of the survivors, there were 393 (71\%) infants who received a stage II procedure, 
which was associated with $17(4 \%)$ further deaths and three (1\%) heart transplants (Schwartz et al. 2014). There was no influence of the type of shunt on mortality post stage II procedure. However, after 12 months of follow-up, the RVPA group had a significantly higher transplant free survival than the BT shunt group. There were 72 events in the RVPA cohort with 68 deaths (25\%), four (1\%) heart transplants, and 100 events in the BT shunt group with 91 (33\%) deaths and nine ( $3 \%)$ heart transplants ( $\mathrm{p}=0.01)$ (Ohye et al. 2010). After three years of follow-up, the transplant-free survival was $67 \%$ for the RVPA group versus $61 \%$ for the BT shunt group $(\mathrm{p}=0.15)$ (Tweddell et al. 2012).

\subsubsection{Risk factors for mortality in the Single Ventricle Reconstruction trial}

\subsection{Pre-Norwood mortality}

The SVR trial investigators examined the preoperative mortality and its associated risk factors using all 921 infants with HLHS screened as part of the SVR trial (Atz et al. 2010). Major extracardiac congenital abnormality (OR 10.0, 95\% CI 4.0-24.7, p<0.0001), gestational age at birth (OR 1.3, 95\% CI 1.1-1.5), $\mathrm{p}=0.02$ ), and presence of obstructed pulmonary venous return (OR 4.0, 95\% CI 1.3-12.3, $\mathrm{p}=0.02$ ) were associated with preoperative death pre-Norwood procedure.

\subsection{Hospital and thirty-day post-Norwood mortality}

The Pediatric Heart Network (PHN) investigators also evaluated the risk factors for Norwood hospitalization mortality and thirty-day post-operative mortality (Tabbutt et al. 2012). They found that lower birth weight (HR 0.62 (95\% CI 0.41-0.93), $\mathrm{p}=0.02$ ), genetic abnormalities (HR 2.89 (95\% CI 1.21-6.9), $\mathrm{p}<0.001)$, receiving extracorporeal membrane oxygenation support (ECMO) post-Norwood procedure (HR 3.41 (95\% CI 1.94-5.98), p <0.001), and having an open sternum on return to the intensive care unit post-Norwood procedure (HR 4.25 (95\% CI 1.6510.6), $\mathrm{p}=0.002$ ) were associated with higher hospital mortality. Similarly, lower birth weight 
(OR 0.52 (95\% CI 0.0.29-0.94), p =0.03), genetic abnormalities (OR 2.93 (95\% CI 0.78-10.9), p $<0.001$ ), receiving ECMO post-Norwood procedure (OR 4.38 (95\% CI 1.76-10.9), p=0.002), and having an open sternum post-Norwood procedure (OR 18.10 (95\% CI 5.17-63.4), p <0.001) were associated with higher thirty-day post-operative mortality. In addition, they found that longer duration of deep hypothermic circulatory arrest (OR 1.10 (95\% CI 1.03-1.19), $\mathrm{p}=0.01)$ was a significant predictor of thirty-day post-operative mortality but was not an important independent risk factor for hospital mortality.

\subsection{Insterstage mortality}

Interstage death is defined as deaths that occur between being discharged home post-Norwood procedure and admission for the stage II procedure. Therefore, this analysis excluded infants kept in hospital from the Norwood procedure to the Stage II procedure. In the SVR trial, 430 (78 $\%$ ) subjects were discharged home following the Norwood procedure but excluded four individuals due to uncharacteristically late stage II procedures (Ghanayem et al. 2012). There were $50(12 \%)$ infants who died during the interstage period and the odds of death in the interstage period were higher in patients who received a BT shunt (OR 3.4, 95\% CI 1.8-6.6, $\mathrm{p}<0.001)$ in the univariable analysis. In the multivariable analysis, shunt type was only an independent risk factor for mortality in patients with mild or no atrioventricular valve regurgitation $(A V V R)(O R$ 9.7, $\mathrm{p}<0.001)$. Other independent risk factors identified in the multivariable analysis were gestational age less than 37 weeks (OR 3.9, 95\% CI 1.4-10.8, $\mathrm{p}=0.008$ ), hispanic ethnicity (OR 2.6, 95\% CI 1.1-6.5, $\mathrm{P}=0.04)$, poverty in the lower tertile compared to the middle tertile (OR 0.15, CI 0.05-0.048), presence of aortic and mitral atresia (OR 2.3, CI 1.1-5.0, p=0.03), and number of post-Norwood procedure complications (OR 1.2, 95\% CI 1.1-1.4, p=0.006) (Ghanayem et al. 2012).

\subsection{Stage II mortality}

There were 17 (4\%) subjects who died from the stage II procedure to hospital discharge and three subjects $(1 \%)$ underwent a heart transplant prior to their hospital discharge (Schwartz et al. 
2014). Univariate comparison between subjects that died or received a heart transplant to the rest of the cohort revealed that patients who died or received a heart transplant had lower weight for age $\mathrm{z}$ score $(-2.5+/-1.6$ vs. $-1.8+/-1.2, \mathrm{p}=0.02)$, higher proportion of non-elective stage II ( $65 \%$ vs. $32 \%, p=0.009$ ), longer total cardiopulmonary bypass time at time of the stage II procedure (134.1 +/- 43.7 min vs. $90.4+/-42.1 \mathrm{~min}, \mathrm{p}<0.001)$, higher proportion of atrioventricular valve repair with the stage II procedure ( $53 \%$ vs. $9 \%$, p $<0.001$ ), higher proportion of patient requiring intervention for arrhythmia ( $12 \%$ vs. $1 \%, \mathrm{p}=0.03$ ) and higher proportion of atrial reduction at the time of stage II procedure ( $3 \%$ vs. $0 \%, \mathrm{p}<0.001$ ).

\subsubsection{Other analyses of risk factors associated with mortality}

\subsection{Hospital and thirty-day post-Norwood mortality}

Furch et al. evaluated risk factors associated with thirty-day post-Norwood mortality in their single center series of 157 infants from 1999 to 2007 (Furck et al. 2010). The thirty-day mortality was $21 \%$ in the first 3 years and $2.5 \%$ in the last three years. The lack of low-flow antergrade selective cerebral perfusion during aortic arch reconstruction (OR 4.85 (95\% CI 1.6114.58)) and an increase of 15 minutes in cardiopulmonary support time (OR 1.41 (95\% CI 1.161.72 per 15 minutes) were independently associated with thirty-day mortality in this group. Anderson \& al evaluated the effect of surgeon and institutional volume on post-Norwood hospital mortality using the Pediatric Health Information System database (Anderson et al. 2016). They looked at hospital mortality not limited to thirty-days post-Norwood and found an hospital mortality of $15 \%$. They found that hospital mortality ranged per center from $7 \%$ to 30 $\%$ (5 th and $95^{\text {Th }}$ centile) and that mortality per surgeon ranged from $0 \%$ to $40 \%\left(5^{\text {th }}\right.$ and $95^{\text {th }}$ centile). They found that after adjusting for patient characteristics, the odds of mortality decreased by $18 \%$ for every 10 additional stage I surgery performed per institution per year (18 $\%$ decrease $(95 \%$ CI $10-26 \%), p<0.001)$. Surgeon volume did not significant affect mortality odds. 


\subsection{Interstage mortality}

A few other studies have also examined interstage death, deaths that occur between being discharged to home post-Norwood procedure and admission for the stage II procedure, and reported an incidence of two to $20 \%$ (Rudd et al. 2014; Hehir et al. 2008; Furck et al. 2010; Ghanayem et al. 2012; Simsic et al. 2004). The Milwaukee group has reported an excellent interstage survival outcome of $98 \%$ for interstage survival using their multidisciplinary home monitoring system (Rudd et al. 2014) while other groups are still reporting significant interstage mortality (Furck et al. 2010; Simsic et al. 2004). A nested case-control study looked at 33 HLHS patients who experienced interstage deaths compared to 99 HLHS survivors (Hehir et al. 2008). This study identified restrictive atrial septal defects (OR 7.6, 95\% CI 1.9-29.6, p=0.003) and an operation age greater than seven days (OR 3.8, 95\% CI 1.3-11. p=0.017) as independent risk factors for interstage death. Another case-control study examined 20 (6\%) patients with interstage mortality from a cohort of 360 patients with HLHS and found that teenage mothers (OR 6.6, 95\% CI 1-9-22.5, p=0.003), single adult caregivers (OR 4.1, 95\% CI 1.2-14.4, p=0.03), a BT or central shunt (OR 4.0, 95\% CI 0.9-18.0, p=0.08), post-operative arrhythmias (OR 2.7, 95\% CI 1.1-6.4, $\mathrm{p}=0.03$ ), and an post-Norwood intensive care unit stay of more than 15 days (OR 2.7, 95\% CI 1.2-6.1, p=0.02) were all independently associated with an increased chance of dying during the interstage period (Taylor et al. 2016). Furck \& al, also evaluated the risk factors associated with interstage death in their single center series of 157 infants with HLHS. Their interstage mortality was $15 \%$ and the only significant idenpendent risk factor for interstage death was a diagnosis of mitral atresia and aortic atresia (OR 4.09 (95\% CI 1.3612.35) (Furck et al. 2010). Finally, the National Pediatric Cardiology Quality Improvement Collaborative also examined interstage mortality (Cross et al. 2014; Schidlow et al. 2015) using information from 33 surgical centers. There were 247 patients that entered the interstage period with $23(9 \%)$ subsequent deaths. The independent risk factors for death were HLHS with aortic stenosis and mitral atresia (RR 12 (95\% CI 6.2-27.3)), HLHS with aortic atresia and mitral stenosis (RR 5.2 (95\% CI 1.0-26.1)), being on anti-seizure medication at discharge to home (RR 12.5 (95\% CI 22.69.2)), gestational age < 34 weeks at birth (RR 11.1 (95\% CI 3.2-37.0)), using a nasogastic or nasojenunal tube for feeding at time of discharge to home post-Norwood procedure (RR5.5 (95\% CI 1.5-20.1)), having any unscheduled readmission to hospital (RR 5.3 (95\% CI 2.8-10.4)), having a primary cardiology identified in the patients' note on less than 50 
\% of clinical visits (RR3.1 (95\% CI 1.6-6.3)), and fewer than two postoperative vasoactive medications used in the post-operative period following stage I procedure (including milrinone, epinephrine, dopamine, dobutamine, norepinephrine, calcium, vasopressin, or nitroprusside) (RR $2.2(95 \%$ CI 1.6-3.2)).

\subsection{Pre-Stage II mortality}

The mortality pre-stage II procedure was also previously assessed independently combining post-Norwood hospital mortality and interstage mortality (Alsoufi et al. 2015). This case-control study followed 219 infants with HLHS post-Norwood procedure. Out of their cohort, 38 patients $(17 \%)$ died while in hospital and 18 patients (10\%) died after hospital discharge but before their stage II procedure. Using a competing risk analysis, they identified postoperative ECMO requirements (HR 3.1, 95\% CI 1.7-5.8, p<0.001), genetic or major extracardiac anomalies (HR 2.5, 95\% CI 1.2-5.2, $\mathrm{p}=0.018)$, unplanned cardiac reoperation (HR2.6, 95\% CI 1.2-5.4, $\mathrm{p}=0.013)$, modified BT shunt (HR 3.4, 95\% CI 2.0-5.9, $\mathrm{p}<0.001$ ), and with a preoperative weight of less than $2.5 \mathrm{~kg}$ (HR 2.5, 95\% CI 1.2-4.1, $\mathrm{p}=0.006$ ) as independent risk factors for death prior to reaching stage II. These studies illustrate the fact that mortality risk and its associated risk factors varies depending on which risk period is analyzed.

\subsubsection{Ventricular dysfunction in HLHS patients}

\subsubsection{Introduction on ventricular dysfunction}

Ventricular dysfunction is very common in patients with HLHS. Ventricular dysfunction can occur at any time in patients with HLHS. It has been described to occur before the Norwood procedure, at the time of the Norwood procedure, at any time during the interstage period, and during the intermediate and long-term follow-up (P. C. Frommelt et al. 2012; G. D. Hill et al. 2015; Wilder, Caldarone, et al. 2015a; D. T. H. Wong et al. 2011; Altmann et al. 2000; Bartram et al. 1997).

\subsubsection{Methods to measure right ventricular function}

The right ventricular function is often estimated visually but can also be measures using multiple 
measurement techniques. The right ventricle does not have a bullet-like shape similar to the left ventricle and the geometry of the right ventricle, especially in the single ventricle population, is variable, making the measurement of right ventricular function more challenging than left ventricular function measurements. The gold standard for right ventricular function measurements is done by cardiac magnetic resonance imaging (MRI), but the two most universally used methods of measurement of right ventricular function by echocardiography are the right ventricular ejection fraction (RVEF) and the right ventricular fractional area of change (RVFAC).

\subsection{Right ventricular fractional area of change}

RVFAC is usually obtained from the transverse apical four-chamber view (Figure 6). RVFAC expresses the percentage change of the calculated right ventricular areas between the enddiastole right ventricular area (the end of the part of the cardiac cycle when the heart relaxes, when the heart is at its biggest) and end-systole right ventricular area (the end of the cardiac contraction, when the heart is at its smallest) (Pavlicek et al. 2011; P. C. Frommelt et al. 2012). RVFAC values of less than $35 \%$ are considered abnormal (Tweddell et al. 2012). RVFAC is calculated based on the following formula:

RV FAC $(\%)=($ right ventricular end-diastolic area - right ventricular end-systolic area $) /$ right ventricular end-diastolic area $\mathrm{x} 100$. 


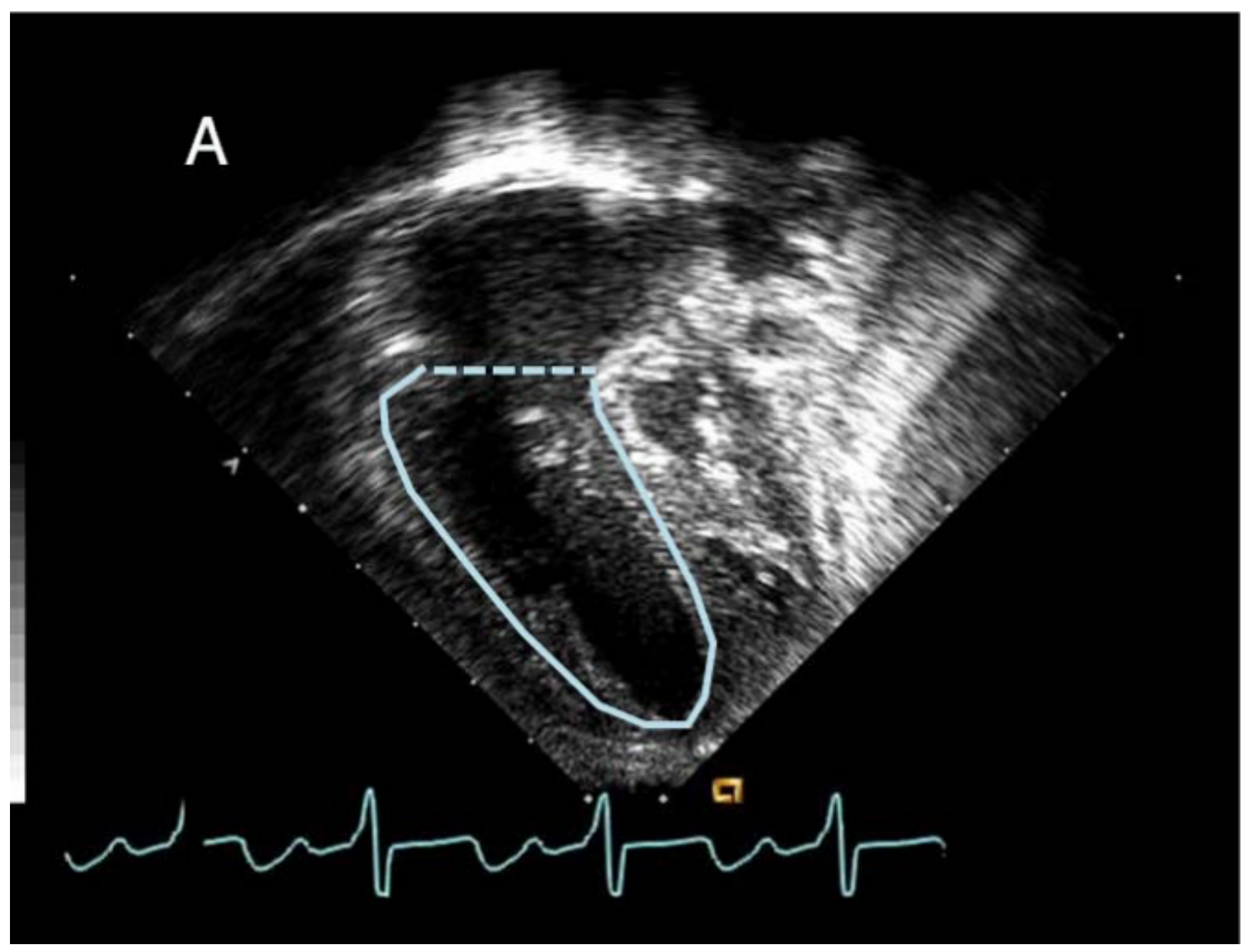

Figure 6. Example of measurement of right ventricular area in transverse apical four-chamber view obtained by echocardiography necessary for calculation of RVFAC and RVEF. This shows the tracing of the right ventricle done to measure the right ventricular area necessary for both RVEF and RVFAC measurements. Reproduce with permission from (Frommelt et al. 2012), Copyright Wolters Kluwer Health Inc. 


\subsection{Right ventricular ejection fraction}

RVEF is similar to RVFAC but expresses the percentage change of volume between end-diastole right ventricular volume (the end of the part of the cardiac cycle when the heart relaxes, when the heart is at its biggest) and end-systole right ventricular volume (the end of the cardiac contraction, when the heart is at its smallest). RVEF is considered abnormal if measured at less than $44 \%$ (Ohye et al. 2008). In the SVR trial (Frommelt et al. 2012), the volumes were obtained from the right ventricular area measured on the transverse apical 4-chamber view at the level of the tricuspid annulus (Figure 6) as well as the right ventricular cavity length measured on the subcostal sagittal view (Figure 7). Then right ventricular volumes were calculated using the biplane pyramidal method (Helbing et al. 1995) and ejection fraction is obtained using the following formula:

(Right ventricular end-diastolic volume - Right ventricular end-systolic volume) / Right ventricular end-diastolic volume X 100 . 


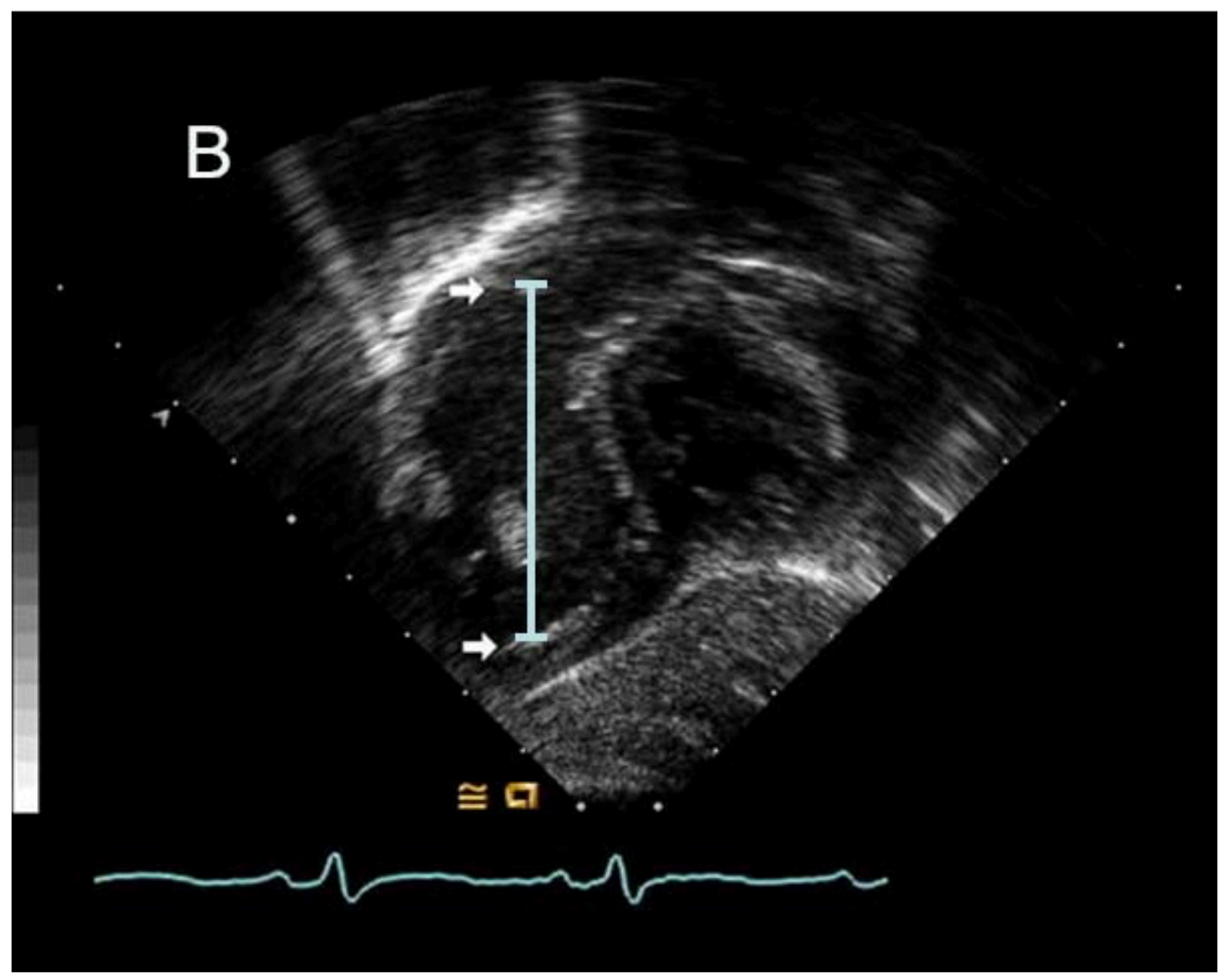

Figure 7. Right ventricular cavity length measured on the subcostal sagittal view from right ventricular apex to pulmonary valve. This shows the right ventricular cavity measurement necessary for RVEF calculation. Reproduce with permission from (Frommelt et al. 2012), Copyright Wolters Kluwer Health Inc.

\subsection{Definition for right ventricular dysfunction by two-dimensional echocardiography}

The gold standard method to measure right ventricular ejection fraction is cardiac MRI and normal values of RVEF measured by MRI are $49 \%$ to $72 \%$ (Pavlicek et al. 2011; Larose et al. 2007). By echocardiography, RVEF is considered abnormal if measured at less than $44 \%$ (Ling \& Marwick 2012). According to the European echocardiography guidelines, RVFAC values are more accurate than RVEF and values less than $35 \%$ are considered abnormal (Lawrence et al. 2010).Therefore, right ventricular dysfunction can be defined by two-dimensional 
echocardiography as having a RVEF measurement of less than $44 \%$ and a RVFAC of less than $35 \%$.

\subsubsection{Incidence of right ventricular dysfunction in HLHS}

In the SVR cohort, the average right ventricle ejection fraction (RVEF) (46\%+/- $9 \%$ ) and area fraction change $(35 \%+/-9 \%)$ were calculated to be low and that throughout the 14 months follow-up (Frommelt et al. 2012). The incidence of moderate right ventricular dysfunction, assessed visually, has been recently shown to peak at three to six months post-Norwood procedure with an incidence of $11 \%+/-3 \%$ (Wilder, McCrindle, et al. 2015b).

\subsubsection{Ventricular dysfunction association with mortality}

Ventricular dysfunction plays a role in survival and morbidity post-Norwood procedure. The volume and pressure loaded single ventricle is subjected to a high workload by perfusing both pulmonary and systemic circulations. This single ventricle is working in a sub-optimal environment with high vascular resistance, high diastolic pressure, and persistent severe cyanosis. In addition, the severity of ventricular dysfunction is a clear risk factor for death in other diagnoses such as dilated cardiomyopathy (Alexander et al. 2013) and myocarditis (Abe et al. 2013). However, the full impact of ventricular dysfunction in patients with HLHS is not fully understood.

\subsubsection{Association of ventricular dysfunction with mortality in the SVR trial}

In the SVR trial analyses, ventricular dysfunction was not always associated with higher risk of mortality. For example, ventricular dysfunction was not an independent risk factor of death or transplant during the pre-Norwood period (Atz et al. 2011), the Norwood hospitalization, the 30 days post Norwood period (Tabutt et al. 2012) and the interstage period (Ghanayem et al. 2012). However, patients that died pre-Fontan procedure had lower mean RVEF (34\%+/- $9 \%$ vs. $43 \%$ 
+/- $7 \%$ ) (Newburger et al. 2014). Therefore, lower RVEF was associated with an increased risk of death or transplant in those patients (HR 1.92 per 5 unit decrease, 95\% CI 1.45-2.50, $\mathrm{p}<0.0001$ ). Similarly, low fractional area of change was an independent predictor of lower transplant-free survival after a mean follow-up of 2.7 +/- 0.9 years (HR 1.49, 95\% CI 1.12-1.97, $\mathrm{p}$ value $=0.006$ ). This demonstrates that ventricular dysfunction does influence mortality over time for patients with HLHS, but might have different degrees of influence depending on the specific time period.

\subsubsection{Association between ventricular dysfunction and mortality in other analyses}

Ventricular dysfunction was also identified as a risk factor for mortality in other analyses. The spike in moderate ventricular dysfunction incidence seen at three to six months post-Norwood was shown to coincides temporally with the early hazard for death or transplant using the Congenital Heart Surgeons' Society cohort (Wilder, McCrindle, et al. 2015b). In a single center series of 138 infants with HLHS, freedom from death or heart transplant was significantly reduced in patients who develop ventricular dysfunction in the early post-operative period after the first stage surgery both at 3 months ( $47 \%$ vs. $84 \%$, p<0.001) and 6 months $(27 \%$ vs. $73 \%$, $\mathrm{p}<0.001$ ) (Chetan et al. 2013). In a cohort study comparing 22 HLHS patients with persistent moderately reduced ventricular function post Norwood procedure to matched control patients with normal function demonstrated a lower freedom from death or transplant at 7 months (53\% vs. $90 \%, \mathrm{p}=0.026$ ) (Jean-St-Michel et al. 2016). A univariate analysis revealed that ECMO or ventricular assist device support after stage I procedure (HR 7.5, 95\% CI 2.4-23.4, $\mathrm{p}=0.0001$ ) and longer duration of inotropic support after stage I discharge (HR 1.014 per day of support, $95 \%$ CI 1.001-1.028, $\mathrm{p}=0.04)$ were predictors of pre-stage II mortality in the group with persistent ventricular dysfunction. Another case-control study examined 20 (6\%) patients with interstage mortality out of a cohort of 360 patients with HLHS and found that depressed ventricular function was associated with an increased chance of interstage death in the univariate analysis (Taylor et al. 2016). As mentioned above, ventricular dysfunction is not always identified as a risk factor for death or transplant in this population. Hehir \& al looked at 33 HLHS patients who experienced interstage deaths compared to 99 HLHS controls (Hehir et al. 
2008) and showed that the incidence of ventricular dysfunction did not differ between the two groups.

\subsubsection{Ventricular dysfunction is not always a permanent diagnosis}

Developing ventricular dysfunction is not necessarily a permanent diagnosis. In other groups of patients such as the dilated cardiomyopathy cohort, myocardial recovery is a well-documented phenomenon (Everitt et al. 2014; Alexander et al. 2013). A subgroup of HLHS patients with moderate to severe ventricular dysfunction, when given enough time, can demonstrate the ability to recover ventricular function and even reach the Fontan completion (Muthurangu et al. 2005; O'Connor et al. 2012). A case-control study looked at 22 patients with persistent moderate ventricular dysfunction following the stage I procedure (Jean-St-Michel et al. 2016). In this cohort, there were six patients with improvement in ventricular function during follow-up and 11 patients were able to proceed to stage II. This is demonstrating that myocardial recovery is possible in this population as well.

\subsubsection{Ventricular dysfunction is a common reason for heart transplant listing}

Despite the lack of understanding of the role of ventricular dysfunction on outcomes, ventricular dysfunction is one of the main reasons for referral for heart transplant assessment. A subanalysis of the SVR trial looked at this question and confirmed that ventricular dysfunction played an important role in the decision to put a patient on the heart transplant waiting list (Kulkarni et al. 2016). They found that lower RVFAC (OR 0.689, 95\% CI 0.543-0.874, $\mathrm{p}=0.002$ ), classic HLHS diagnosis (OR 0.362, 95\% CI 0.136-0.966, p 0.042) as well as longer intensive care unit (ICU) days post Norwood procedure (OR 2.416, 95\% CI 1.572-3.713, p<0.001) were independently associated with listing for heart transplant. They also demonstrated that the survival for HLHS patients listed for heart transplant was lower than for the main cohort, with a waiting list mortality of $39 \%$ and an overall mortality, including both waiting list and post-heart transplant mortality, of $52 \%$ at one-year post listing. In addition, this analysis also provided information on this population's ability to wait for a heart transplant with median time to heart transplant of 25 
days (IQR 2-271 days) and median time to death of 128 days (IQR 10-707 days). This information is of utmost important in the current situation of organ shortages and long wait times. The Pediatric Heart Transplant Study group also studied this population and found that the independent risk factors for mortality while waiting were younger age (HR 1.03, 95\% CI 1.021.04, $\mathrm{p}$ value) and higher status at listing, meaning being sicker at time of entering the waiting list (status 1A) (HR 3.81, 95\% CI 1.43-10.21, $\mathrm{p}=0.008$ ) (Carlo et al. 2016). This reflects that sicker and younger patients are more likely to die prior to receiving a heart transplant. Early identification of who needs to be listed for heart transplant and a better understanding of cumulative risk factors for death for those with ventricular dysfunction could reduced the waiting list mortality and increase the chance for those children to receive a heart. Early identification of the need to be listed for heart transplant would allow the clinicians to list the patients earlier, before they are too sick to survive the wait period. This is of utmost importance in order to improve the chances of those patients to receive a heart transplant. 


\section{Chapter 2 \\ Rationale}

Despite the lack of clarity regarding what impact ventricular dysfunction has on outcomes in patients with HLHS, ventricular dysfunction is a main reason for heart transplant listing within this patient group. The reality is that with their unstable and unique physiology, ventricular function influences, to some degree, morbidity and mortality in patients with HLHS. However, ventricular dysfunction has a large spectrum of severity, is not always easy to quantify, and is not always a permanent diagnosis. With current long wait times for heart transplant and associated high wait list mortality, clinicians need to list patients for heart transplant as early as possible. Ultimately, clinicians must decide which patient is fit to continue down the surgical path and which patient is not. In the situation when the surgical palliation fails, heart transplant or palliative care are often the only viable options. Due to this, we wanted to assess risk factors for death in this already high-risk patient population, infants with HLHS, and ventricular dysfunction post-Norwood procedure.

Our main objective was to identify the patient and procedural factors, both modifiable and nonmodifiable, present at the post-Norwood echocardiogram that would be associated with the inability to proceed to stage II palliation in the group of HLHS patients with right ventricular dysfunction. We asked that, for patients with HLHS and ventricular dysfunction, what patient characteristics representing both modifiable and non-modifiable risk factors are associated with the inability to proceed to stage II palliation, defined as death or heart transplantation without achieving stage II surgery? 


\section{Chapter 3}

\section{Methodology}

\subsection{Study design}

This study is a prospective cohort study. We performed a secondary analysis of the information that was collected as part of the SVR trial. The SVR trial was a non-blinded randomized surgical trial, which had enrolled 555 infants with HLHS from 14 different centers across the United States and Canada and randomized the patients between two surgical procedures leading to two different sources of pulmonary blood flow (BT shunt vs. RVPA conduit) (Ohye et al. 2008; Ohye et al. 2010). The PHN, a consortium of clinical sites that conducts research studies in children with congenital or acquired heart disease, performed this trial. The PHN has performed multiple other clinical studies in the past.

\subsection{Patient population}

\subsubsection{SVR trial study cohort}

We selected our study cohort from the 555 infants enrolled in the SVR trial. The NHLBI Pediatric Heart Network SVR Trial dataset was used in the preparation of this work (Ohye, Sleeper, et al. 2010; Ohye et al. 2008). The data were downloaded from https://www.pediatricheartnetwork.com/pud_login.asp?study_id=SVR on 04/01/2016. As part of the SVR trial, the infants were screened between May 2005 and July 2008 in 14 centers across the United States and Canada. In order to be included in the SVR trial, infants required a diagnosis of HLHS or a related single morphologically right ventricle anomaly, had a Norwood surgical procedure (stage I procedure) planned without an anatomical reason to prefer one specific pulmonary shunt type (BT shunt vs. RVPA conduit) over the other, and without other major congenital abnormalities. Infants were randomly assigned to one surgical procedure (BT 
shunt vs. RVPA conduit) using randomly permuted blocks within strata defined by the presence of aortic atresia and the presence of obstructed pulmonary venous return. For the purpose of the SVR trial, aortic atresia was diagnosed using a 2-dimensional echocardiography and defined as no blood flow through the aortic valve. The presence of obstructed pulmonary venous return was defined by the use of specific interventions done after birth such as an atrial septostomy or an urgent surgical Norwood procedure.

\subsubsection{Protocolized echocardiogram in the SVR trial}

All 555 infants enrolled in the SVR trial were screened to see if they met our eligibility criteria for our study cohort (secondary analysis). The infants enrolled in the SVR trial underwent echocardiograms on a preset schedule, which included the following time points: before the Norwood procedure (baseline), post-Norwood procedure, pre-stage II, and 14 months of age. For this secondary analysis, we defined ventricular dysfunction if it was present on the postNorwood echocardiograms, which were done after the Norwood procedure at a mean age of 22.5 +/-13.4 days post-Norwood procedure (Frommelt et al. 2012).

There are multiple measurements that can provide a clinical understanding of the ventricular function of a patient. However, measuring right ventricular function accurately is more difficult than measuring left ventricular function because the right ventricle is not bullet shaped and its geometry varies (Lang et al. 2015). Right ventricular function is often estimated visually by looking at the heart beating using 2-dimensional echocardiography. However, the SVR trial echocardiogram protocols were very detailed (Ohye 2008). All patient echocardiograms documented multiple specific measurements of right ventricular function as well as multiple other echocardiographic measurements. The two most universally used measurements of RVEF and RVFAC were utilized in the SVR trial. In addition, in the context of the SVR trial, echocardiograms were done and interpreted in a rigorous way in a core laboratory. All 14 centers received training prior to trial commencement on the chosen echocardiographic protocols, and all studies were centrally measured offline at a core laboratory at the Medical College of Wisconsin, 
initially by a trained pediatric echocardiography technician and then reviewed by an experienced pediatric cardiologist.

\subsubsection{Right ventricular function measurements}

The gold standard method to measure right ventricular ejection fraction is cardiac magnetic resonance imaging (MRI) and normal values of RVEF measured by MRI are $49 \%$ to $72 \%$ (Pavlicek et al. 2011; Larose et al. 2007). Unfortunately, MRI was only performed on a subgroup of patients for the SVR trial. However, the SVR trial investigators did perform a study comparing RVEF values obtained by cardiac MRI and RVEF obtained by two-dimensional echocardiography for the subgroup of patients who underwent both measurement techniques, and demonstrated a good agreement between the two measurements (Frommelt et al. 2012). By echocardiography, RVEF is considered abnormal if measured at less than $44 \%$ (Ling \& Marwick 2012). According to the European echocardiography guidelines, RVFAC values are more accurate than RVEF and values less than $35 \%$ are considered abnormal (Lawrence et al. 2010). Right ventricular dysfunction can be defined by two-dimensional echocardiography as having a RVEF measurement of less than $44 \%$ and a RVFAC of less than $35 \%$. In the SVR trial, the agreement between cardiac MRI measurements and two dimensional echocardiography measurements was only assessed for RVEF. In addition, RVFAC measurements have been demonstrated to be superior to RVEF measurements to assess right ventricular function with echocardiography. Therefore, we opted to use both RVEF and RVFAC to define right ventricular dysfunction for selection of our cohort. We hoped that by requiring both measurements for entry into the study cohort, we would only select patients who truly have right ventricular dysfunction reducing the bias from inappropriate selection of controls, a form of selection bias.

\subsection{Inclusion and exclusion criteria}

In order to be selected into our study cohort, patients were required to be enrolled in the SVR trial and to have a post-Norwood echocardiogram that showed an RVEF of less than $44 \%$ and an RVFAC of less than $35 \%$. Patients with missing values for RVEF or RVFAC on the post- 
Norwood echocardiogram, without a post-Norwood echocardiogram or with an echocardiogram coded as unacceptable for analysis by the core laboratory, were excluded from the study. In addition, patients with no event (transplant, death or stage II procedure) at the 12-month followup visit were also excluded since this is uncharacteristic for patients with HLHS and most likely represents a group of patient with a different anatomical substrate.

\subsection{Human Research consideration}

The dataset we used was provided by the PHN, had been completely de-identified, and is publically accessible via the Pediatric Heart Network website. We consulted the SickKids Research Ethics Board and were informed that no Research Ethics Board approval was required.

\subsection{Outcome measures}

The primary outcome was death or heart transplant without achieving stage II palliation. Therefore, there were three possible competing risk events: death without achieving stage II, heart transplant without achieving stage II, and stage II. However, for the purpose of this analysis, and because there were only two subjects who underwent a heart transplant, death and heart transplant were analyzed as one composite outcome.

\subsection{Data collection}

Multiple variables were collected during the SVR trial and these included baseline characteristics, anatomical characteristics, Norwood hospitalization characteristics, unintended cardiovascular interventional procedures, information with complications, genetic information, and site/surgeon information. For this analysis, we identified multiple candidate predictors that would be available to the clinician at the time of the post-Norwood discharge echocardiogram. We analyzed multiples variables from the baseline demographic, the patients' characteristics 
prior to the Norwood and Norwood hospitalization characteristics. We also evaluated parameters from the baseline echocardiogram and the post-Norwood echocardiogram. Please see the list of candidate variables in Table 1.

\subsection{Sample size}

Preliminary analysis demonstrated that 123 patients would fulfill our inclusion criteria. If we estimated a similar or higher proportion of death or transplant in the ventricular dysfunction cohort compared to the entire SVR cohort, we would expect to have at least 30 deaths or heart transplant events for the study cohort. Knowing these estimates, we planned that our final model would include three to four predictive variables, therefore around 10 events per predictive variables.

\subsection{Statistical Methodology}

\subsubsection{Descriptive statistics}

Descriptive statistics were performed for all variables, assessing distribution, completeness, biologic plausibility, miscoding, and outliers. Patients with missing values or coded as unacceptable quality for the post-Norwood RVEF and RVFAC measurements were excluded from the cohort. Binary variables were created for death or heart transplant outcomes prior to the stage II procedure (yes/no). Complications at baseline and post-Norwood procedure were documented as text variables in the SVR dataset. Types of complications were combined in order to create a series of binary variables (baseline and post-Norwood procedure cardiac complications, respiratory complications, neurological complications, gastrointestinal complications, infectious complications, renal complications, and others). The right ventricular dysfunction cohort and the rest of the SVR cohort with normal right ventricular function were compared for the differential occurrences for all predictor variables. Categorical variables were 
reported as frequencies and percentages and compared using chi-square tests. Continuous variables were reported as means and standard deviations for normally distributed variables, medians, and interquartile ranges (IQR) for non-normally distributed variables. Comparisons for continuous variables were performed using student' t-tests for normally distributed predictors and Wilcoxon rank-sum tests for non-normally or skewed distributed or count variables. All descriptive statistical analyses were performed using SAS 9.4 software, and an alpha (type 1 error) threshold of less than 0.05 for all statistical tests was employed.

\subsubsection{Data imputation}

Missing data were reported at less than $2 \%$ per variables (Frommelt et al. 2012) in the SVR dataset. However, we expected that some variables would have a higher percentage of missing values. We excluded from the analysis the continuous variables with more than $20 \%$ of missing values. We also excluded categorical variables associated with less than five events, in order to avoid model over specification. A multiple imputation algorithm was then used to impute missing data for the remainder of the variables with missing data. We created five imputed dataset using the MIANALYZE procedure in SAS, which represents a random sample of the missing data by selecting a random sample of values within the original dataset. Descriptive statistics were performed for all imputed variables in order to assess distribution, biologic plausibility, and miscoding of the imputed dataset. We imputed the mean to replace the few outliers that were not biologically plausible post imputation, such as a negative ventricular volume.

\subsubsection{Time to event analysis}

Our study cohort was selected based on measured right ventricular function on the post-Norwood echocardiogram. However, the timing of that echocardiogram was variable and multiple factors, both patient and environmental, influenced the timing of this test, such as patient clinical stability, technician availability, echocardiogram laboratory availability, overall acuity of the ICU, timing of the Norwood procedure, etc. The risk of death and heart transplant as well as the 
risk of developing ventricular dysfunction will start immediately post-Norwood procedure. Therefore, the period of observation may be artificially shortened because a patient might develop ventricular dysfunction a long time before the post-Norwood echocardiogram and, therefore, this period of time would not be included into our time to event analysis (Figure 8). This phenomenon is called left censoring, and is defined when the event of interest takes place at an unknown time prior to when the individual is being observed. No other method of selection was possible, because of the information available to us within the SVR dataset. We did not have access to other measurements of ventricular function between the baseline echocardiogram and the post-Norwood echocardiogram. Due to this, we adjusted for left censoring by using an existing methodology employing parametric survival analysis developed by Blackstone et al (Blackstone et al. 1986). We included the risk period prior to the post-Norwood echocardiogram, which was the period of time from the Norwood procedure to the post-Norwood echocardiogram. In other words, the time to death, heart transplant, or stage II was defined as the time from the Norwood procedure to the post-Norwood echocardiogram plus the time from postNorwood echocardiogram to the event (Figure 9). By making a distribution assumption on the added risk period, we adjusted for left censoring reducing the bias associated with left censoring (Cain et al. 2011). 

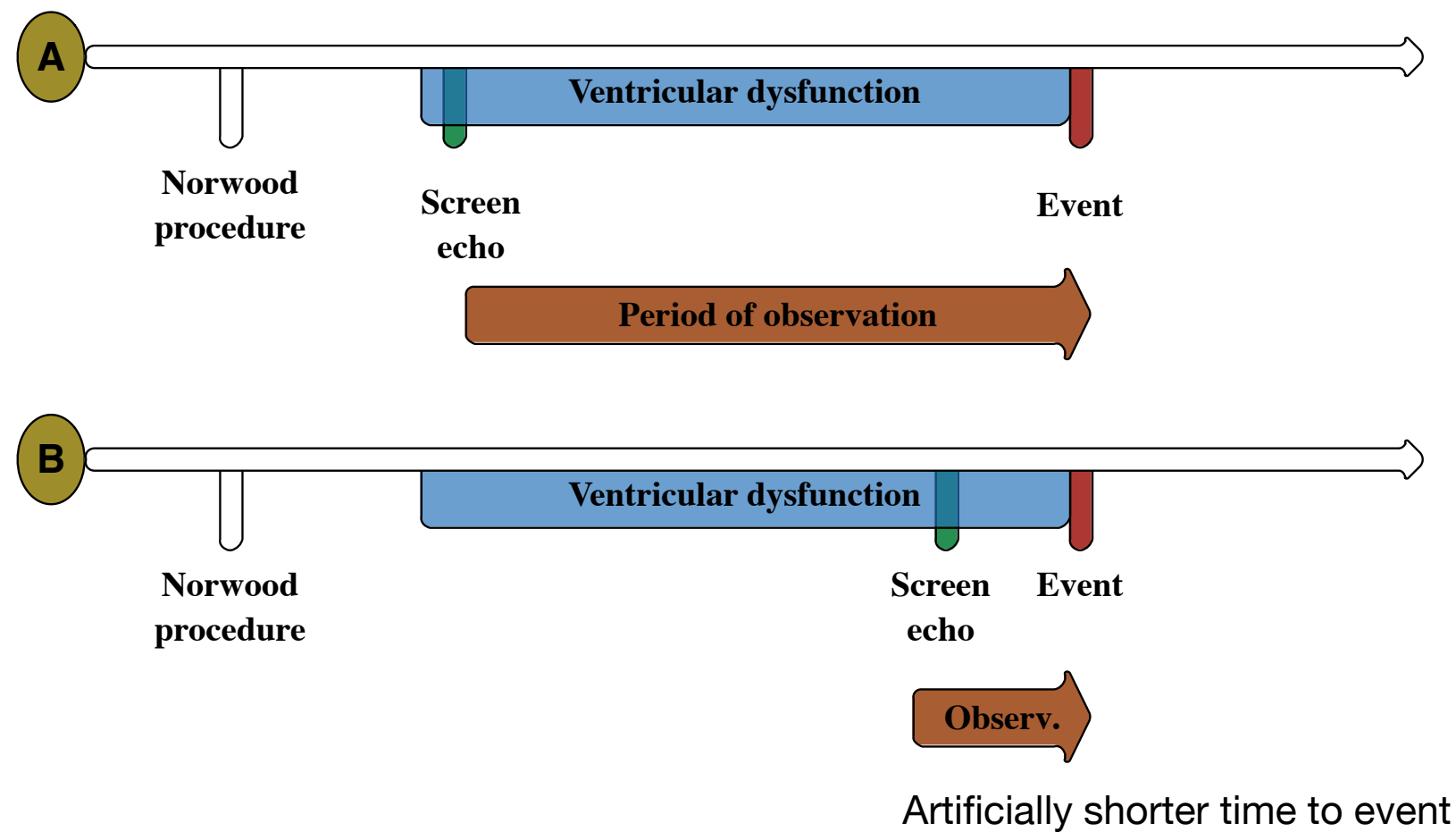

Figure 8. Left censoring. A) This scenario represents a patient who is diagnosed with right ventricular dysfunction on the screening post-Norwood echocardiogram soon after developing ventricular dysfunction. The real time to event is observed. B) This scenario represents a patient who develops right ventricular dysfunction but has the screening post-Norwood echocardiogram much later. Therefore, the time to event for this patient is artificially shorten. 


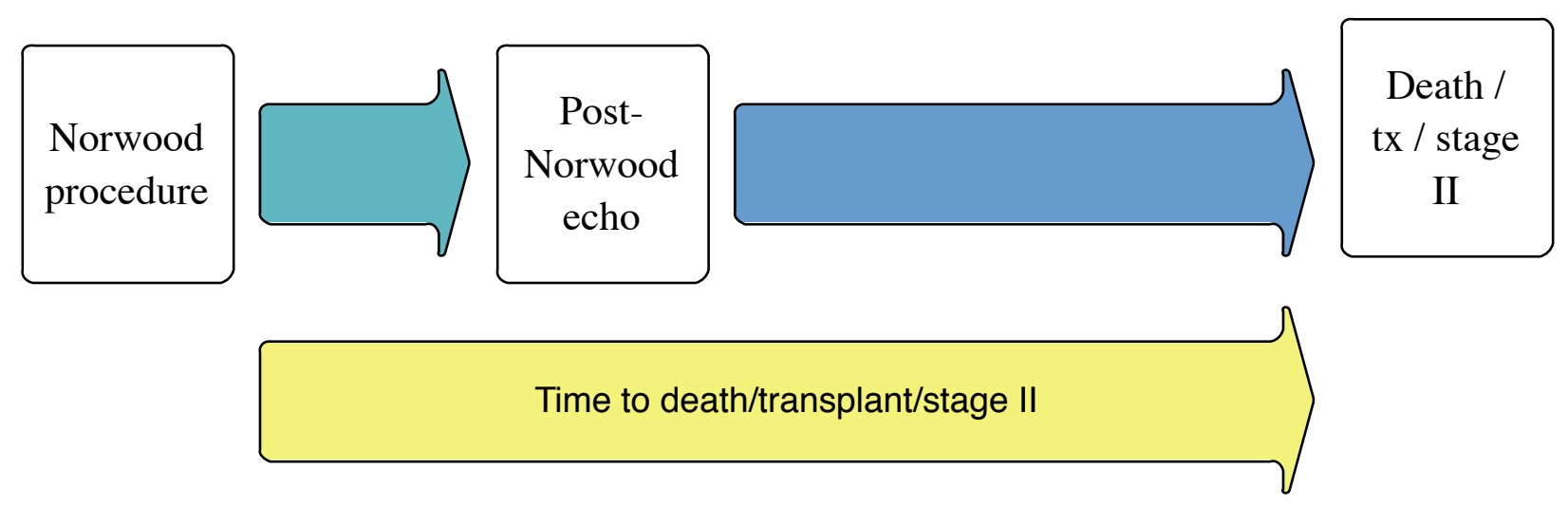

Figure 9. Time to death, heart transplant, or stage II. The time to death, heart transplant or stage II was defined as the time from the Norwood procedure to the post-Norwood echocardiogram plus the time from post-Norwood echocardiogram to the event. This graph represent the observation period included in our analysis after adjusting for left censoring.

\subsubsection{Censoring}

Since we are studying the ability to progress to stage II, we censored patients at the stage II procedure. Our events of interest were death without achieving stage II or heart transplant without achieving stage II. In this dataset, all 123 patients underwent either one of the three outcomes (death prior to stage II, heart transplant prior to stage II, or stage II procedure). As a result, no patients were lost to follow-up and none ended the trial before reaching an event. Therefore, we had no patients that were "right censored" but left censoring is relevant to our analysis and has been discussed above in the "Time to event analysis" section.

\subsubsection{Hazard modeling}

\subsubsection{Model the hazard phases}

We chose to explicitly model the hazard function with a multivariable parametric proportional hazard modeling technique using the method developed by Blackstone and colleagues 
(Blackstone et al. 1986). In other words, we decomposed the cumulative hazard function into phases of time-varying risk. With this analysis technique, we hoped to achieve an increased statistical power, prediction of survival time, and potentially provide more insight about the clinical and biological processes involved. This method used the maximum likelihood in order to decompose the time to event into a maximum of three phases of time-varying risk (Sergeant, Blackstone \& Meyns 1997; Sergeant et al. 1997). Non-parametric Kaplan-Meier plots were constructed to guide the goodness of fit of the parametric models. From the cumulative hazard function, up to three individual hazard phases were identified and decomposed, as previously described by Blackstone, et al (Blackstone et al. 1986). The three possible phases are the early phase, which represents decelerating risk period; the constant phase, which represents a hazard phase with constant hazard risk; and, finally, a late phase, which represent an accelerating risk period (Figure 10). The phases describe the variation of hazard overtime but do not describe the timing of those phases. For example, the early phase does not have to start early and the late phase does not have to occur late in the period of observation. 

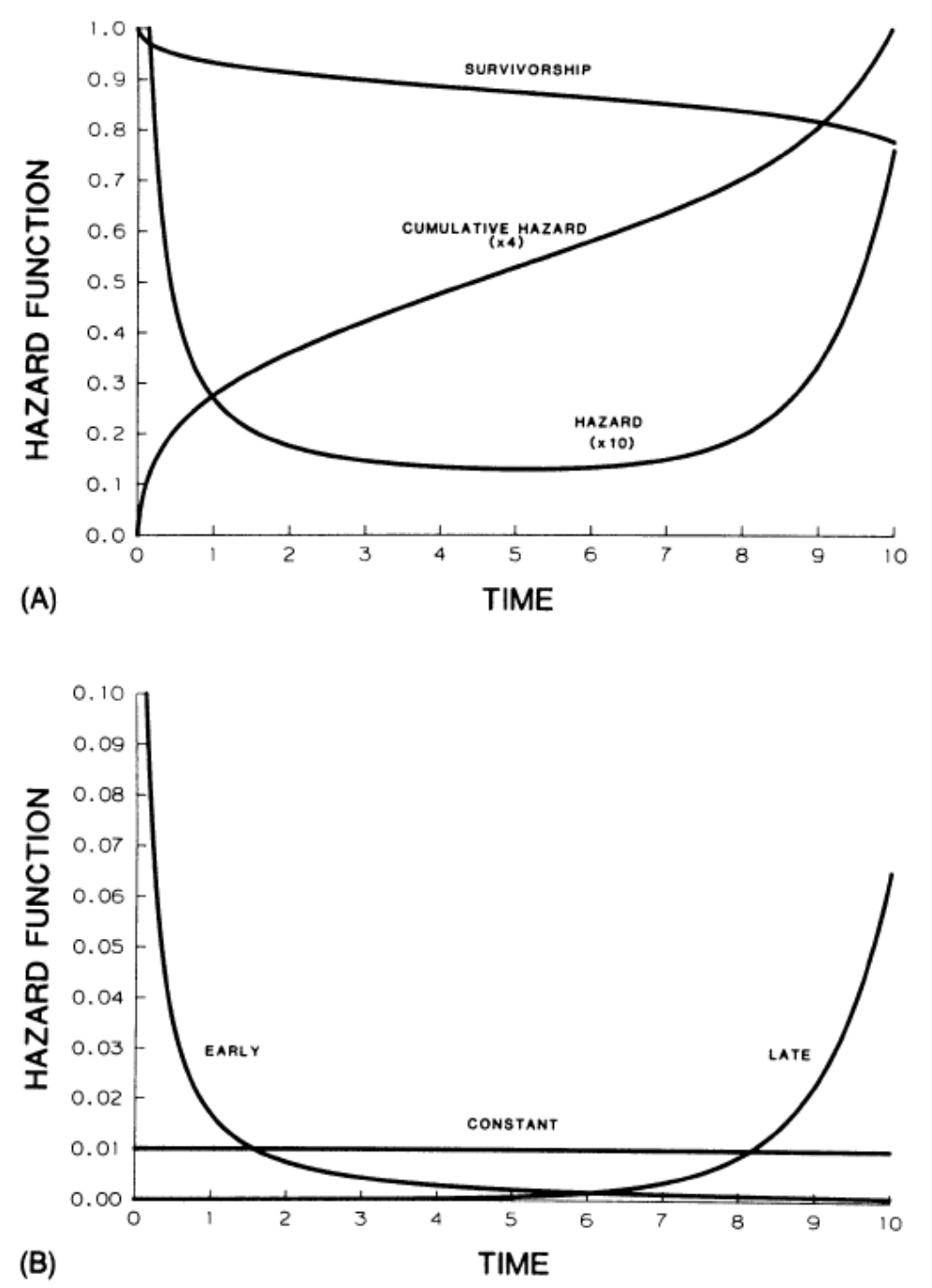

Figure 10. The functions used to describe time related events with parametric modeling. The panel A graphically represents the cumulative hazard function, which accelerates rapidly initially before reaching a constant slope and then rising again, explaining the change in the survivorship function. Finally, the corresponding hazard function is infinite at time zero and then is constant at a low level to rise again. The panel B depicts possible decomposition of the hazard function into three separate risk periods labeled here as early, constant, and late. This graph represent the variation in risk associated with each phases. The three possible phases are the early phase, which represents decelerating risk period; the constant phase, which represents a hazard phase with constant hazard risk; and, finally, a late phase, which represent an accelerating risk period. Reproduce with permission from (Blackstone \& al, 1986), Copyright Taylor \& Francis Informa UK LTD. 


\subsubsection{Goodness of fit of the parametric model}

We attempted modeling all three phases both individually and combined. The form of the equation for each phase was determined using cumulative hazard function graphs. Most models were expected to be unable to fit the data and error messages to be obtained. We then graphically assessed the goodness of fit of the possible models by plotting the non-parametric Kaplan-Meier estimates and the independent maximum likelihood estimate of the parametric models while demonstrating the associated $70^{\text {th }}$ confidence interval of the parametric models and the $95^{\text {th }}$ confidence interval of the Kaplan-Meier estimates. The $70^{\text {th }}$ confidence interval was used in order to have a more rigorous visual assessment of the goodness of fit of our parametric model. Once the best fitting model was identified, the actual parameters of the formula describing the distribution of time were used in the multivariable model and the final model. All parametric survival analyses were performed using SAS 9.2 since the macro created by Blackstone and colleagues was not available for the most recent version of SAS.

\subsubsection{Identification of associated factors}

Variable selection was planned to be done for each identified hazard phase separately, since each hazard phase is independent of the others. In order to avoid over specification of the model and to identify risk factors associated with mortality or heart transplant, we needed to employ variable selection techniques. Bootstrapping with stepwise auto selection was then used to guide variable selection using a cut-off $\mathrm{p}$ value of 0.12 to enter the model and a 0.1 cut off to stay in the model (Austin \& Tu 2004). Bootstrap samples composed of 123 observations were sampled five hundred times and a stepwise variable selection was performed on each bootstrap sample. Predictor variables that were found in at least $25 \%$ of the bootstrap models were maintained in the model (reliability threshold). The risk factors retained after performing the bootstrapping with stepwise autoselection were then placed into the model and subjected to a backward selection, with a retention threshold of $\mathrm{p}<0.05$. Predictor variables that were found with a $\mathrm{p}$ value of less than 0.05 were retained in the final model. 


\subsubsection{Nature and influence of risk factors}

The resulting model from the variable selection represents the final overall model. To explore the influence of the individual retained factors, we constructed parametric prediction plots using the formula from the final multivariable parametric model (Sergeant, Blackstone \& Meyns 1997). Using this technique, we were able to construct nomograms representing the parametric equations for specific supplied values for the variables. For example, in order to represent the hypothetical average patient, the mean values for all risk factors were specified in the model equation. In addition, we could maintain all values constant, except for one risk factor, and individually assess the effect of all identified categorical risk factors. To improve our insight on the role the continuous variable risk factors played, we calculated the cumulative hazard at one time point in follow-up for the average hypothetical patient. We chose six months for clinical reasons, since most children will undergo the stage II by four to six months of age. We then graphically depicted the transplant-free survival at six-months post-Norwood procedure (y axis) versus the continuous variable (x axis). To help understand the hypothetical average patient, we wanted to graphically represent the cohort average survival predictions. We employed a similar technique but, this time, we averaged the calculated prediction plots for all 123 patients in our ventricular dysfunction cohort. Using the real values of the variables for each 123 patients, we created prediction plots for all individual patients within the cohort. We then presented the averages in one graph. Using this method, we could again graphically represent the influence of all the identified risk factors by averaging subgroups of patients with or without a specific risk factor and compare them visually. Similar to the hypothetical average analysis, in order to improve our insight on the role the continuous variable risk factors played, we calculated the cumulative hazard at 6 months post Norwood procedure for all the patients of our ventricular dysfunction cohort using their specific risk factor values. We then graphically depicted the transplant-free survival at six-months post-Norwood procedure (y axis) versus the continuous variable (x axis). 


\subsubsection{Best and worst-case scenario predictions}

Finally, in the hope to assist clinical decisions, we solved our model equation in order to predict survival for the patient with and without the risk factors. Using the best and worst values for each of the independent risk factors identified, we predicted survival for all the different scenarios. In order to improve our understanding of the impact of shunt type in this subgroup of patients, we also stratified the best and worst case predicted survival by shunt type.

\subsubsection{Assumptions and model verification}

The assumptions of non-parametric survival modeling do not all apply to parametric hazard modeling. In parametric hazard modeling, hazard is decomposed into phases of risk and, therefore, does not need to be constant over time. Random censoring, however, does apply to parametric hazard modeling. As a randomized clinical trial, significant effort was applied to retain patients within the trial. As a result, all patients received follow-up and none ended the trial before reaching an event. Each variable was tested for outliers and missing data so we could assume the absence of influential observations. Absence of influential outliers was also assessed though casewise diagnostics, examining patterns and common influential values on visual inspection of DFbetas and CookD. Over-specification was assessed through a lack of convergence warning of the model or inappropriate $95 \%$ confidence intervals for parameter estimates. To verify our model, we solved our multivariable parametric final model equation for all the patients within the ventricular dysfunction cohort. Then we graphed the average of all the predicted survival curves with its $70 \%$ confidence intervals. Following this, we superimposed the Kaplan-Meier estimates with their $95 \%$ confidence intervals on the graph. The $70^{\text {th }}$ confidence intervals were used in order to have a more rigorous visual assessment of the goodness of fit of our parametric model. To assess the goodness of fit of our model, and if there was a residual risk not covered by our model, we visually evaluated if the Kaplan-Meier estimates, with their respective confidence intervals, fell within the $70 \%$ confidence interval of our cohort average parametric solution for our cohort. 


\section{Chapter 4}

\section{Results}

\subsection{Characteristics of the cohort}

\subsubsection{Identification of the study cohort}

There were 555 infants enrolled in the SVR trial between May 2005 and July 2008. Of those, five subjects died while in the operating room during the Norwood procedure and were excluded from the cohort (Figure 11). Of the 550 patients left, 118 (22\%) had missing or marked as "unacceptable" RVEF calculated values on the post-Norwood echocardiogram. There were 25 (5 $\%$ ) infants with missing or marked as "unacceptable" RVFAC calculated values and also had missing RVEF values. Therefore, 368 infants out of the 555 (66\%) enrolled patients could be selected for the ventricular dysfunction cohort. One subject who had ventricular function information with normal right ventricular function had no other information collected such as outcome, baseline information, surgical, or post-surgical information, and was rightfully excluded from the analysis. One subject with normal ventricular function received the stage II procedure at 701 days post randomization, which does not follow the usual clinical pathway for this patient population and is most likely due to a different anatomical substrate. Again, this led to the patient being excluded from the analysis. Similarly, one subject with normal ventricular function did not reach any of the outcomes by one year, resulting in exclusion from the analysis as it most likely represented a different anatomical substrate. After study exclusion, we were left with $365(66 \%)$ infants in the study cohort (Figure 11). 


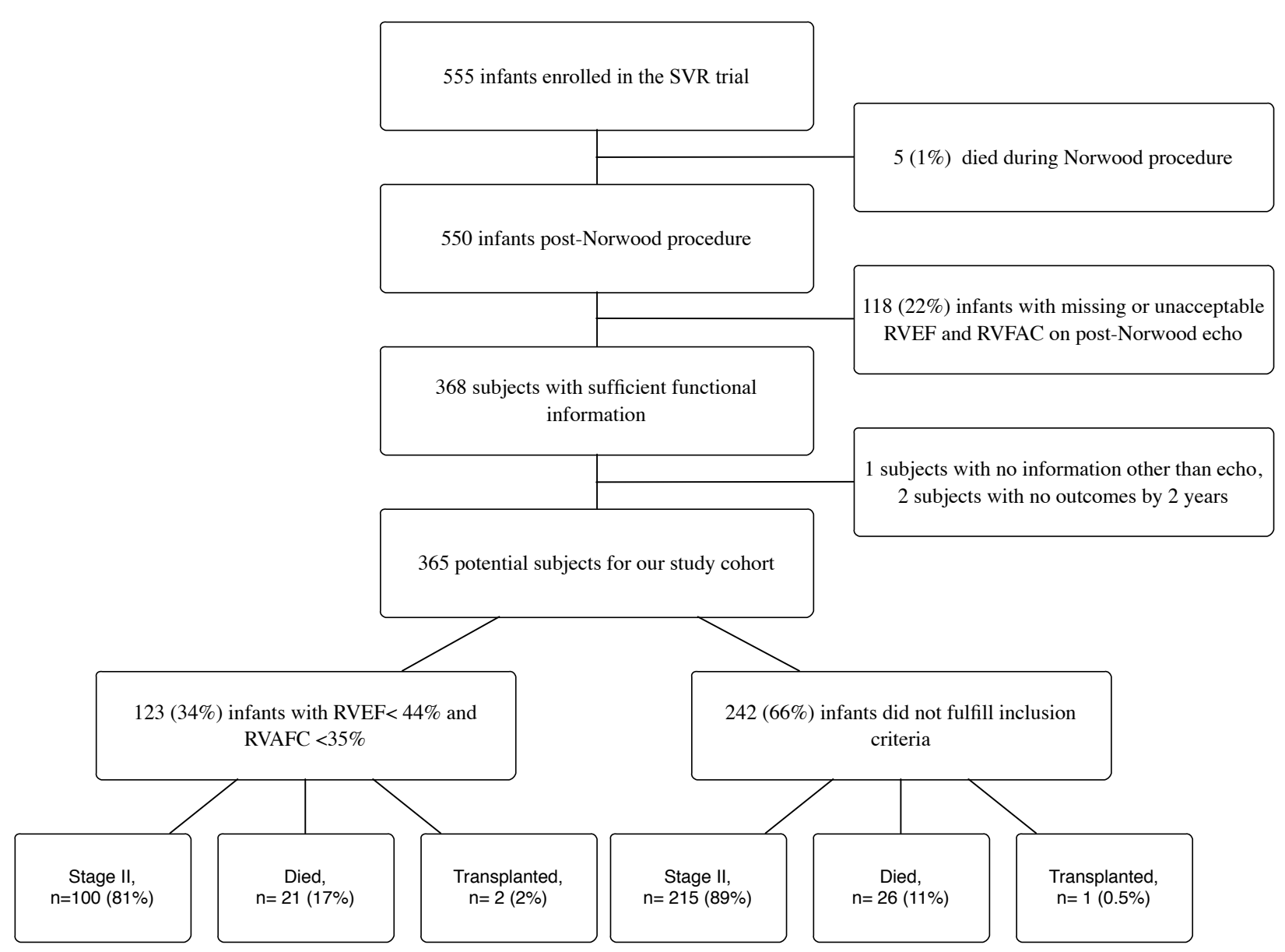

Figure 11. Study subjects flow chart. This demonstrate how the study cohort was selected.

\subsubsection{Characteristics of the right ventricular dysfunction cohort}

Of the 365 infants, 123 (34 \%) subjects had an RVEF of less than $44 \%$ and an RVFAC of less than $35 \%$. This formed our ventricular dysfunction cohort (Figure 11). Of the 123 infants with ventricular dysfunction, 67 (54\%) were male, with a mean birth weight of 3163.1 grams +/525.1 grams, and a median gestational age of 38 weeks (IQR 38-39 weeks). There were 111 (90 $\%)$ infants with usual HLHS anatomy with either mitral valve stenosis or atresia and either aortic valve stenosis or atresia (Table 2). There were 33 (27\%) infants who had complications prior to the Norwood procedure. There were 83 subjects $(67 \%)$ randomized to the modified BT shunt arm, receiving a modified BT shunt at the time of their Norwood procedure and 40 (33\%) subjects were randomized and received an RVPA conduit. The median age at the Norwood 
procedure was six days (IQR 4-8 days). The median ICU stay was 13 days (IQR 9-26 days) with a median hospital length of stay of 24 days (IQR 15-40 days). After the Norwood procedure, 10 (8\%) patients were supported on ECMO and $22(18 \%)$ infants received cardiopulmonary resuscitation (CPR). The median duration of ventilation was seven days (IQR 5-13 days) and 96 (78 \%) infants had their sternum open after leaving the operating room post-Norwood procedure. The median number of complications was two (IQR 1-4) with 56 (46\%) subjects having cardiac complications, 53 (43\%) with respiratory complications, seven (6\%) with neurological complications, 29 (24\%) with gastrointestinal complications, 11 (9\%) with renal complications, and $50(41 \%)$ with infectious disease complications. There were $118(96 \%)$ subjects able to be discharged from hospital after the Norwood procedure without receiving a heart transplant. The median oxygen saturation at discharge post-Norwood procedure was $82 \%$ (IQR 79-85\%) with nine (7\%) subjects requiring supplemental oxygen. Patients were discharged on a median of five (IQR 4-7) medications with 49 (40\%) individuals on an angiotensin-converting-enzyme (ACE) inhibitor, two $(2 \%)$ on a beta-blocker, six $(5 \%)$ on inotropic medications, $102(83 \%)$ on diuretics, four (3\%) on anti-arrhythmic medications, and five (4\%) on anti-hypertensive medications. In this cohort of patients with ventricular dysfunction, 100 (81\%) subjects were able to proceed to the stage II procedure, 21 (17\%) died without achieving the stage II procedure, and two (2\%) patients underwent a heart transplant without achieving the stage II procedure. One of the two patients who underwent a heart transplant prior to stage II procedure died two days after receiving the heart transplant.

\subsubsection{Baseline echocardiogram results for the right ventricular dysfunction cohort}

From the results from the baseline echocardiogram for the ventricular dysfunction cohort (Table 3), 49 (32\%) infants had a patent aortic valve, 23 (19\%) had a patent mitral valve, and 85 (69 $\%)$ infants had a visible left ventricle on their 2-dimensional echocardiogram. The median ascending aortic size was $0.3 \mathrm{~cm}$ (IQR $0.2-0.5 \mathrm{~cm}$ ) with a median left pulmonary artery dimension of $0.41 \mathrm{~cm}(I Q R$ 0.35-0.47 cm) and a median atrial septal defect Doppler gradient of $5.0 \mathrm{mmHg}$ (IQR 2.1-8.2 $\mathrm{mmHg}$ ). There were seven (6\%) infants with aortic valve regurgitation and 34 (28 \%) with pulmonary valve (also called neoaorta) regurgitation. In addition, 91 (74 \%) 
subjects had tricuspid valve regurgitation but only 17 (14\%) infants had a grade of moderate or greater. The median right ventricular end-diastolic long axis dimension was $3.0 \mathrm{~cm}$ (IQR 2.8-3.2 $\mathrm{cm}$ ) with a median short axis dimension of $2.4 \mathrm{~cm}$ (IQR 2.0-2.7 cm). The median anteroposterior tricuspid valve annulus was $1.22 \mathrm{~cm}$ (IQR $1.11-1.39 \mathrm{~cm}$ ) with a median z score of +1.7 (IQR 0.82.9). Similarly, the median transverse tricuspid valve annulus was $1.34 \mathrm{~cm}$ (IQR $1.19-1.52 \mathrm{~cm})$ with a median $z$ score of +1.7 (IQR 1.0-2.6). The median RVEF on the baseline echocardiogram was $45 \%$ (IQR 40 - $49 \%$ ) with a median RVFAC of $33 \%$ (IQR 28 - $37 \%$ ). There were no infants able to fulfill the inclusion criteria with the baseline echocardiogram measurements of RVEF and RVFAC. In other word, there was no infant with RV dysfunction on the baseline echocardiogram based on our definition. There were $23(19 \%)$ subjects with mitral regurgitation and the median left ventricular ejection fraction was $26 \%$ (IQR $9-44 \%$ ).

\subsubsection{Post-Norwood echocardiogram results for the right ventricular dysfunction cohort}

At the time of the post-Norwood echocardiogram, the median RVEF was $39 \%$ (IQR 36 - $42 \%$ ) with a median RVFAC of $30 \%$ (IQR 26 - $33 \%$ ) (Table 4). However, this represented a worsening of RVEF for 61 (50\%) infants (Figure 12) and a worsening of RVFAC for 76 (62\%) infants from the baseline measurements (Figure 13). The median right ventricular end-diastolic dimension was $3.0 \mathrm{~cm}$ (IQR 2.7-3.2 cm). The median right ventricular indexed end-diastolic volume was $99.6 \mathrm{~mL} / \mathrm{m}^{2}$ (IQR $83.3-118.1 \mathrm{~mL} / \mathrm{m}^{2}$ ) with a right ventricular indexed end-diastolic area of $23.3 \mathrm{~cm}^{2} / \mathrm{m}^{2}$ (IQR $20.7-26.9 \mathrm{~cm}^{2} / \mathrm{m}^{2}$ ). The median anteroposterior tricuspid valve annulus was $1.36 \mathrm{~cm}$ (IQR 1.2-1.56 cm) with a median z score of + 2.4 (IQR 1.5-3.6). Similarly, the median transverse tricuspid valve annulus was $1.45 \mathrm{~cm}$ (IQR $1.30-1.60 \mathrm{~cm}$ ) with a median $\mathrm{z}$ score of +2.2 (IQR 1.3-3.0). In this cohort, 110 (89\%) infants had tricuspid valve regurgitation with 26 infants had a moderate grade and six infants had a severe grade. There were 44 (11\%) infants for whom the tricuspid valve regurgitation worsened between the baseline echocardiogram and the post-Norwood echocardiogram (Figure 14). Finally, only six (5\%) subjects had aortic valve regurgitation, while 59 (48 \%) had pulmonary valve regurgitation (also called neoaortic valve) and 28 (23\%) had mitral valve regurgitation. 


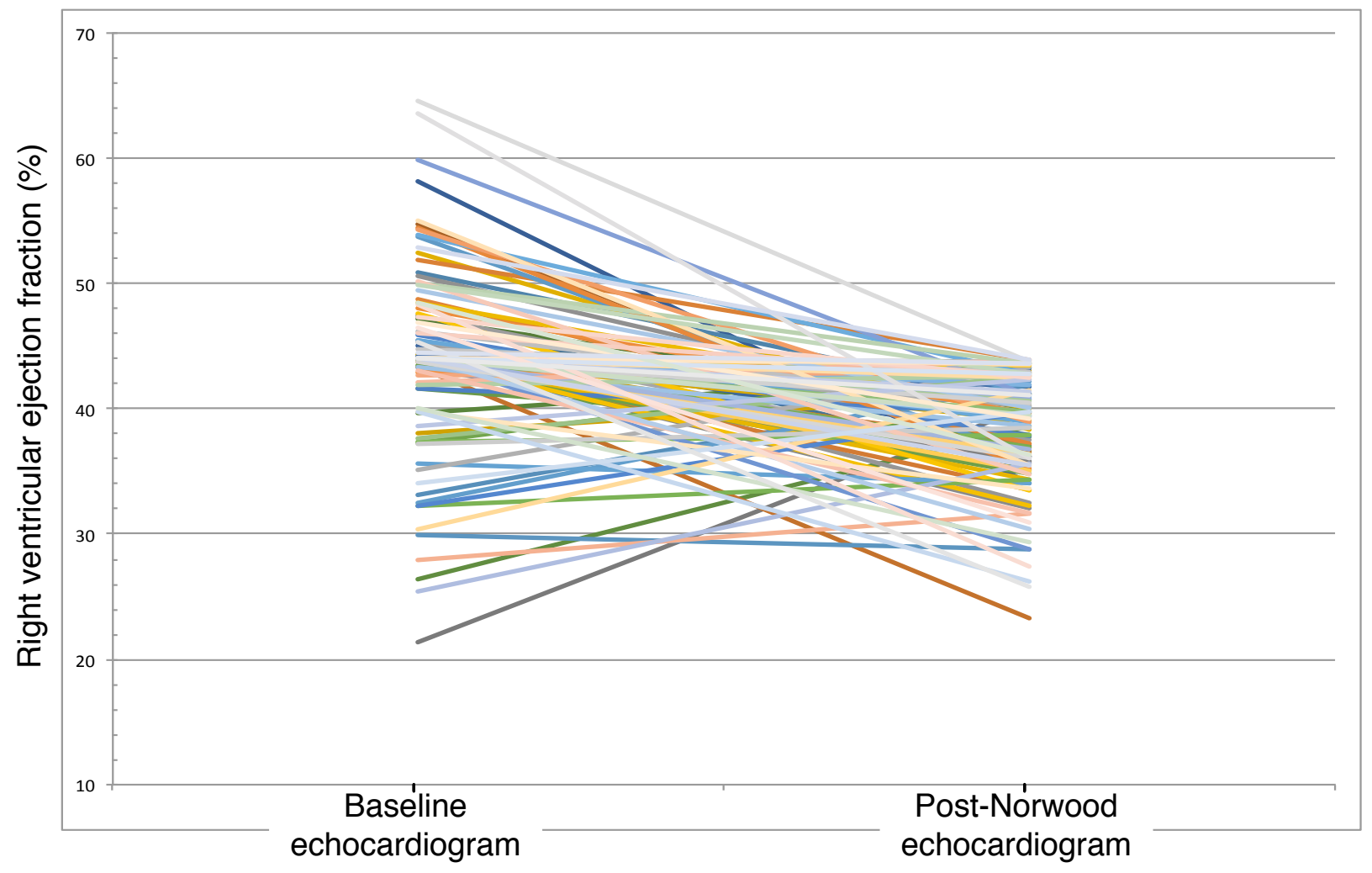

Figure 12. Changes in RVEF measurements between the baseline and the postNorwood echocardiogram. This graph is showing changes RVEF measurements from the baseline and post-Norwood echocardiograms for all 123 patients within the right ventricular dysfunction cohort. 


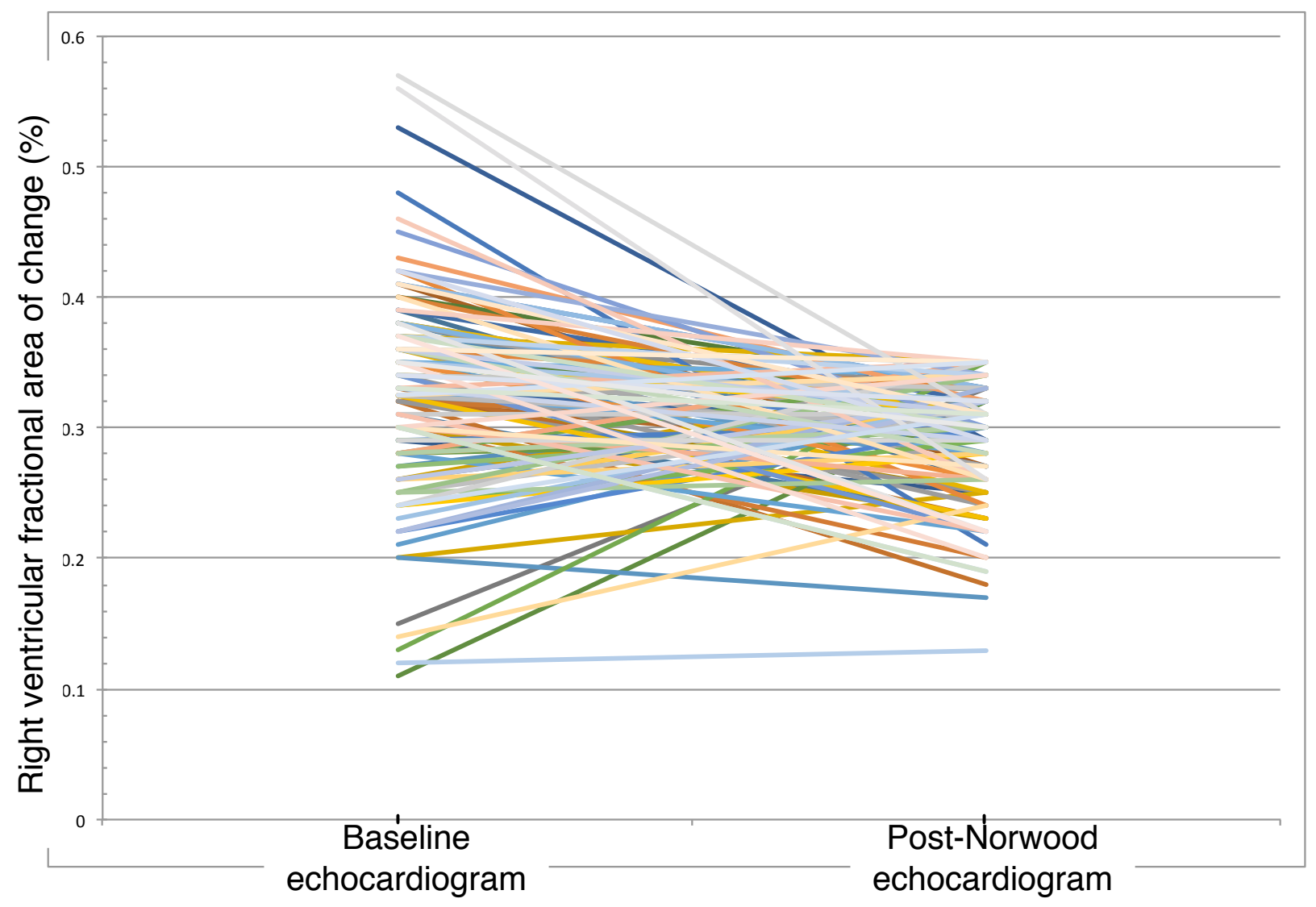

Figure 13. Changes in RVFAC measurements between the baseline and the postNorwood echocardiogram. This graph is showing changes RVFAC measurements from the baseline and post-Norwood echocardiograms for all 123 patients within the right ventricular dysfunction cohort. 
Baseline echocardiogram

Post-Norwood echocardiogram

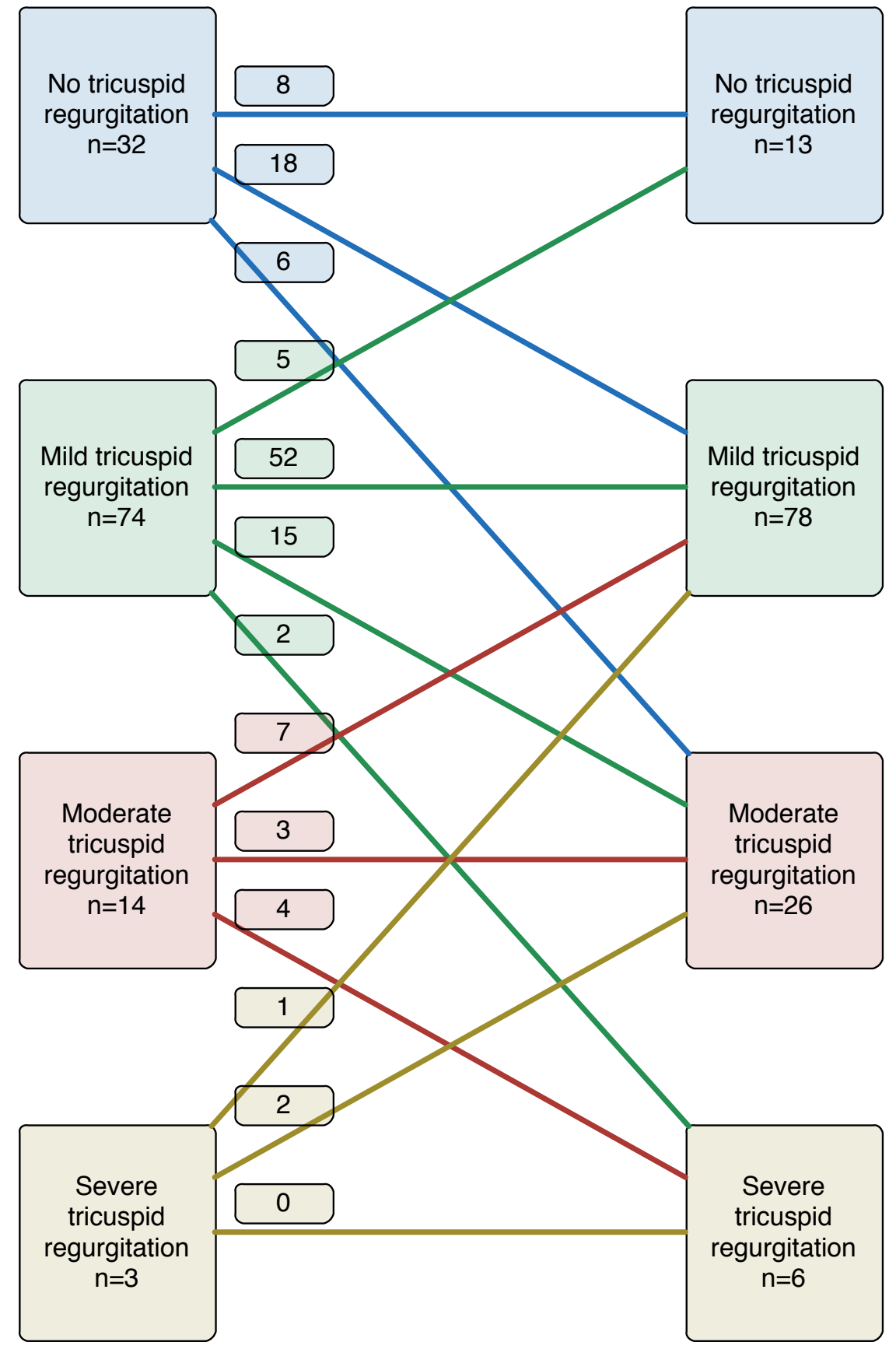

Figure 14. Change in tricuspid valve regurgitation severity between the baseline and the post-Norwood echocardiogram. This graph demonstrating the variation in the severity of tricuspid regurgitation between the baseline and post-Norwood echocardiograms for all 123 patients of the right ventricular dysfunction cohort. 


\subsubsection{Descriptive statistics}

The following variables had more than $20 \%$ of missing data and were excluded from the risk factor analysis (presence of fetal intervention, highest lactate pre-Norwood, regional cerebral perfusion time and flow, ultrafiltration after bypass, size (diameter and length) of BT and RVPA shunt and type of alpha-blockade used) (Table 1). Similarly, some echocardiographic variables on both the baseline and post-Norwood echocardiogram were removed from the risk factor because they had more than $20 \%$ missing values (left ventricular ejection fraction, left ventricular size and function, mitral, neoaortic and aortic valve regurgitation, tissue doppler variables, right ventricular isovolumic time, calculated volume of right ventricle) (Table 1). We also excluded the following variable from the risk factor analysis since less than five subjects had these risk factors (presence of a right subclavian artery, presence of a partial or total anomalous venous drainage, presence of pre-Norwood respiratory, neurological, gastrointestinal, infectious disease complications as well as being discharged on beta-blocker, inotrops, antiarrhythmic medication or anti-hypertensive medications). Otherwise, there were no duplicate participant identifiers, invalid dates, or biologically implausible values.

\subsection{Comparison of the ventricular dysfunction cohort to the normal right ventricular function cohort}

\subsubsection{Comparison of the patient characteristics}

We compared characteristics of the cohort with right ventricular dysfunction $(n=123)$ to the rest of the cohort with normal ventricular function $(n=242)$ before multiple imputation of missing data was performed (Table 2). Of the 365 infants, 242 (66\%) infants did not fulfill the inclusion criteria of a RVEF measurement of less than $44 \%$ and a RVFAC measurement of less than $35 \%$ on the post-Norwood echocardiogram (Figure 11). The right ventricular dysfunction cohort had significantly smaller proportion of male patients $(67(56 \%)$ vs. $157(65 \%), p=0.05)$, a higher

proportion of patients who received ECMO support pre-Norwood procedure (9 (7 \%) vs. 4 (2 
$\%$ ), $\mathrm{p}=0.01$ ), as well a higher proportion randomized to receive the modified BT shunt (83 (67 $\%)$ vs. $85(35 \%), p=<0.0001)$. In addition, the total cross clamp time was shorter (47 min (IQR 36-62 min) vs. $54 \mathrm{~min}$ (IQR 40-66 $\mathrm{min}$ ), p=0.03), a higher proportion of patients received steroids prior to the operation (115 (93\%) vs. $206(85 \%), \mathrm{p}=0.02)$ and a smaller proportion received aprotinin during the operation $(86(70 \%)$ vs. $196(81 \%)$ p=0.01). Finally, more patients with ventricular dysfunction received CPR post-Norwood procedure (22 (18\%) vs. 20 (8\%), $\mathrm{p}=0.007$ ) and a higher proportion of patients with dysfunction had cardiac complications postNorwood procedure $(56(46 \%)$ vs. $80(33 \%), p=0.02)$. The transplant-free survival to the stage II procedure, analyzed using non-parametric Kaplan-Meier, was significantly lower for the right ventricular dysfunction cohort on inspection and the log rank test of quality chi-square was 4.23 with an associated $\mathrm{p}$ value of 0.04 (Figure 15). 


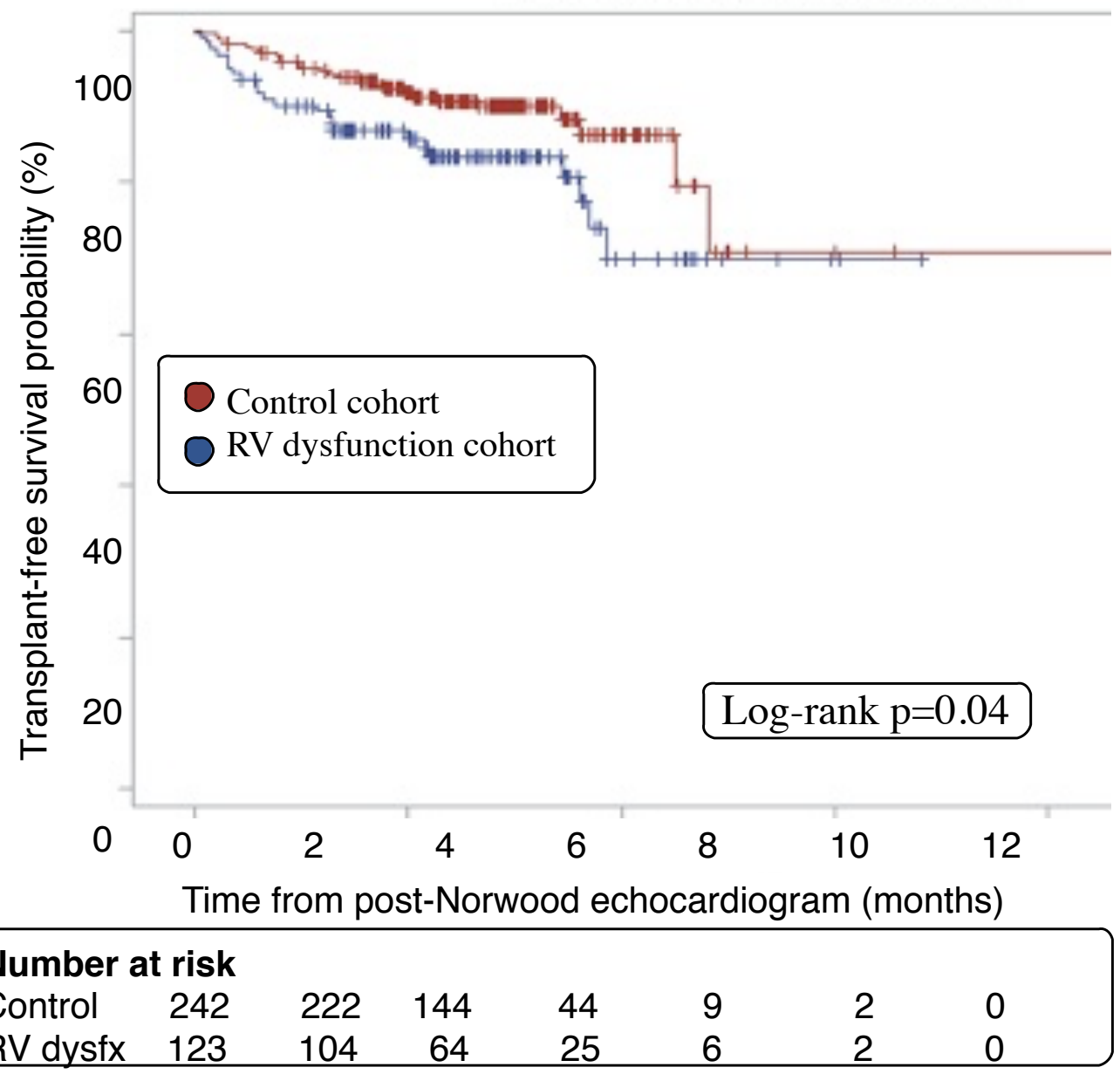

Figure 15. Non-parametric Kaplan-Meier curves for freedom from death or transplant to stage II procedure stratified by cohort. The blue line represents the patients with right ventricular dysfunction $(\mathrm{n}=123)$ and the red line represents the normal function cohort $(\mathrm{n}=242)$, the patients that did not fulfill our inclusion criteria.

\subsubsection{Comparison of the baseline echocardiogram measurements}

On the baseline echocardiogram (Table 3), the ventricular dysfunction group already had a lower median RVEF (45 \% (IQR 40 - 49 \%) vs. $48 \%$ (IQR 42 - $54 \%$ ), p0.002) and lower median RVFAC (33\% (IQR $28-37 \%$ ) vs. $36 \%$ (IQR $31-42 \%$ ), p $<0.001$ ). However, no subjects in either cohort were able to fulfill the inclusion criteria (RVEF $<44 \%$ and $\operatorname{RVFAC}<35 \%$ ) on the 
baseline echocardiogram. The ventricular dysfunction patients also had higher median right ventricle end-diastolic dimensions ( $2.4 \mathrm{~cm}$ (IQR 2.0-2.7 cm) vs. $2.2 \mathrm{~cm}$ (IQR 1.9-3.0 cm), $\mathrm{p}=0.01$ ) measured in the short-axis but no difference in end-diastolic dimension measured in long axis (3.0 cm (IQR 2.8-3.2 cm) vs. $3.0 \mathrm{~cm}$ (IQR 2.7-3.2 cm), p=0.18). However, there was no significant difference for tricuspid regurgitation and tricuspid valve size.

\subsubsection{Comparison of the post-Norwood echocardiogram measurements}

On the post-Norwood echocardiograms (Table 4), the tricuspid size was significantly larger in the ventricular dysfunction cohort as indicated by the median anteroposterior tricuspid valve annulus size (1.36 cm (IQR 1.2-1.56 cm) vs. $1.29 \mathrm{~cm}(\mathrm{IQR} 1.17-1.44 \mathrm{~cm}), \mathrm{p}=0.01)$ and $\mathrm{z}$ score $(+2.4$ (IQR 1.5-3.6) vs. $+2.0(\mathrm{IQR} 1.1-3.1), \mathrm{p}<0.01)$ as well as indicated by the transverse tricuspid valve annulus size (1.45 cm (IQR $1.30-1.60 \mathrm{~cm})$ vs. $1.38 \mathrm{~cm}$ (IQR $1.22-1.57 \mathrm{~cm}$ ), $\mathrm{p}<0.01)$ and the tricuspid valve area $\left(159.4 \mathrm{~cm}^{2}\right.$ (IQR $\left.127.2-185.0 \mathrm{~cm}^{2}\right)$ vs. $144.1 \mathrm{~cm}^{2}$ (IQR 113.9-170.6 $\mathrm{cm}^{2}$ ), $\left.\mathrm{p}<0.01\right)$. However, there was no difference for the presence of tricuspid valve regurgitation and its severity. Similarly, there were no differences regarding the proportion of patients with pulmonary valve (also called neoaortic valve) regurgitation, aortic valve regurgitation and mitral valve regurgitation. In addition, there was no difference in end-diastolic dimension measured in long axis $(3.0 \mathrm{~cm}$ (IQR 2.7-3.2 cm) vs. $3.0 \mathrm{~cm}$ (IQR 2.7-3.2 cm), $\mathrm{p}=0.16)$.

\subsection{Association of shunt type with ventricular function}

Within our cohort selected for the presence of abnormal ventricular function at the postNorwood echocardiogram, shunt type was not associated with different ventricular function measurements. RVEF measurements done on the post-Norwood echocardiogram were not significantly different between shunt type groups (RVEF: 38 \% (IQR 36 - $42 \%$ ) with BT shunt vs. 39\% (IQR $36-43 \%$ ) with RVPA, p=0.35). Similarly, RVFAC measurements done on the post-Norwood echocardiogram were also not significantly different between shunt type groups (RVFAC: 29 \% (IQR 26 - 32 \%) with BT shunt vs. 30 \% (IQR 29 - $33 \%$ ) with RVPA, p=0.25). 
The right ventricular end-diastolic size measured on the post-Norwood echocardiogram was also not significantly different between shunt type groups (3.01 cm (IQR 2.80-3.23) with BT shunt vs. $2.96 \mathrm{~cm}$ (IQR 2.80-3.32) with RVPA, $\mathrm{p}=0.43$ ). The right ventricular end-diastolic size did not differ between patients who had tricuspid regurgitation on the post-Norwood echocardiogram versus patients who did not ((3.09 cm (IQR 2.69-3.19) vs. $3.01 \mathrm{~cm}$ (IQR 2.81-3.20), p=0.81). Finally, patients with at least moderate tricuspid valve regurgitation did not have bigger enddiastolic measurements of the right ventricle than patients without moderate tricuspid valve regurgitation (2.89 cm (2.76-3.09) vs. $3.03 \mathrm{~cm}(\mathrm{IQR} 2.83-3.25), \mathrm{p}=0.11)$.

\subsection{Risk-unadjusted parametric survival analysis for the ventricular dysfunction cohort}

\subsubsection{Left censoring}

Left censoring can bias survival analysis results when a condition or a state is observed but the investigators do not know when the period of risk started (Clark et al. 2003). The ventricular dysfunction cohort was selected based on results of the post-Norwood echocardiogram. The infants were at risk of developing ventricular dysfunction and at risk of dying or receiving a heart transplant starting immediately after the Norwood procedure. The timing of this echocardiogram was not done via a specific protocol and, therefore, could be influenced by multiple patient and non-patient factors. The time between the Norwood procedure and the post-Norwood echocardiogram for our cohort with ventricular dysfunction was variable with a median time of 15 days (9-22 days) (Figure 16). As a result, the time to death and time to heart transplant would be artificially longer or shorter depending on the timing of the post-Norwood echocardiogram and not because of patient factors (Figure 8). In addition, the fact that no patients in our study cohort had right ventricular dysfunction on the baseline echocardiogram based on our inclusion criteria confirmed our choice of risk period. 
The magnitude of the effect of left censoring on our results would likely be small only if the immediate post-Norwood period is a low-risk period for mortality or heart transplant. Since the post-Norwood period may likely be a high-risk period, we needed to adjust for left censoring in our analysis. We adjusted for left censoring by using a methodology employing parametric survival analysis developed by Blackstone et al (Blackstone et al. 1986) allowing us to make distribution assumptions of the added risk period. We then included the risk period prior to the post-Norwood echocardiogram, which is the period of time from the Norwood procedure to the post-Norwood echocardiogram (Figure 9) and then by making distribution assumptions on this added risk period, we adjusted for left censoring reducing the bias associated with this (Cain et al. 2011).

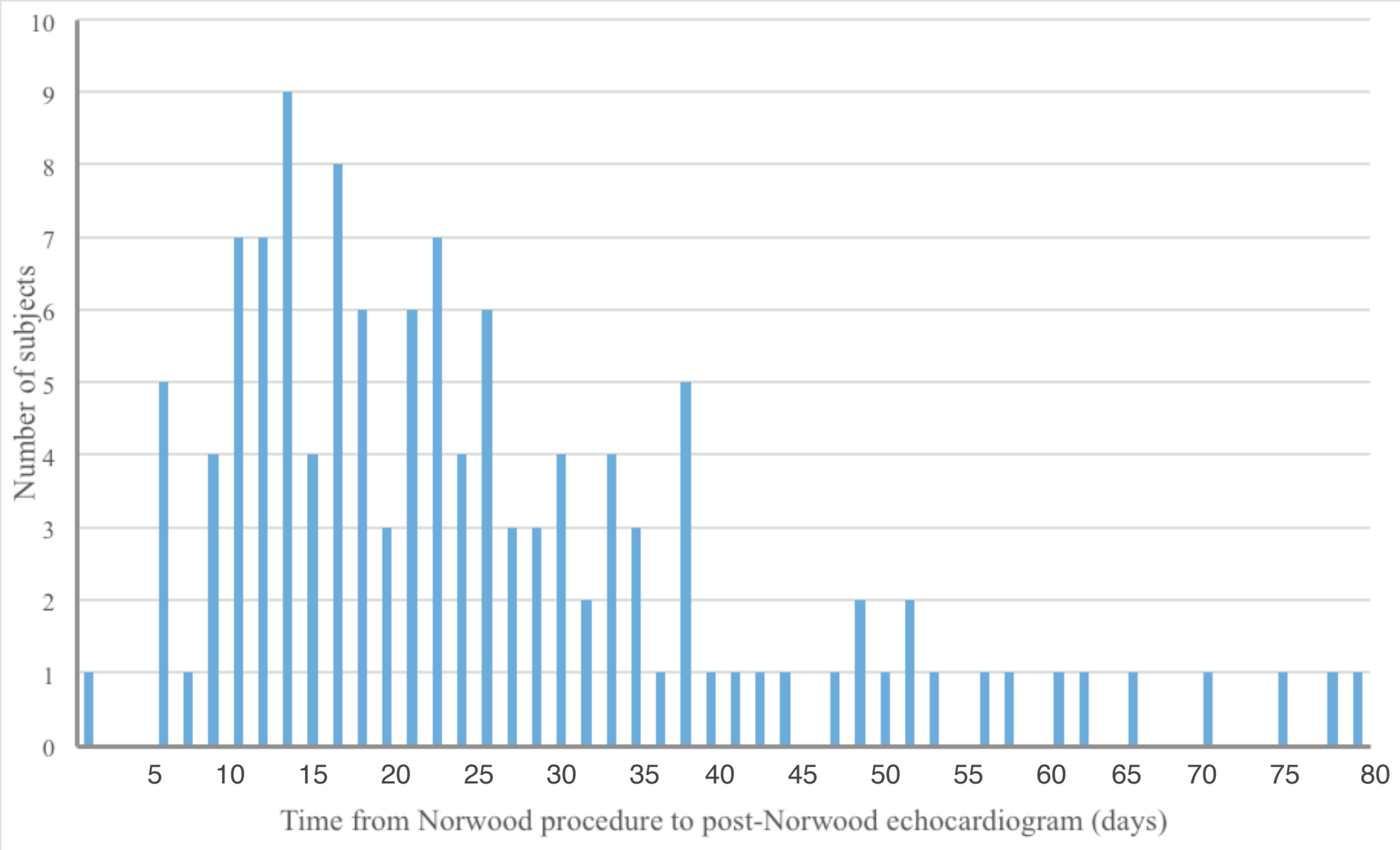


Figure 16. Distribution of time in days from the Norwood procedure to the postNorwood echocardiogram.

\subsubsection{Hazard modeling}

From the cumulative hazard function, we identified only one risk phase. In addition, only a single constant hazard phase and a single late phase model were able to fit the data without obtaining error messages. We then graphically assessed the goodness of fit of the constant phase and late phase models by plotting the non-parameteric Kaplan-Meier estimates and the independent maximum likelihood estimate of the constant phase parametric model and the late phase parametric model while demonstrating the associated $70^{\text {th }}$ confidence interval of parametric models and the $95^{\text {th }}$ confidence interval of the Kaplan-Meier estimates (Figure $17 \&$ 18). Then we identified that the data were best fitted using a constant phase only model (Figure 17) and the parameters of this model were used for the multivariable risk factor analysis as well as specifying the final model. 


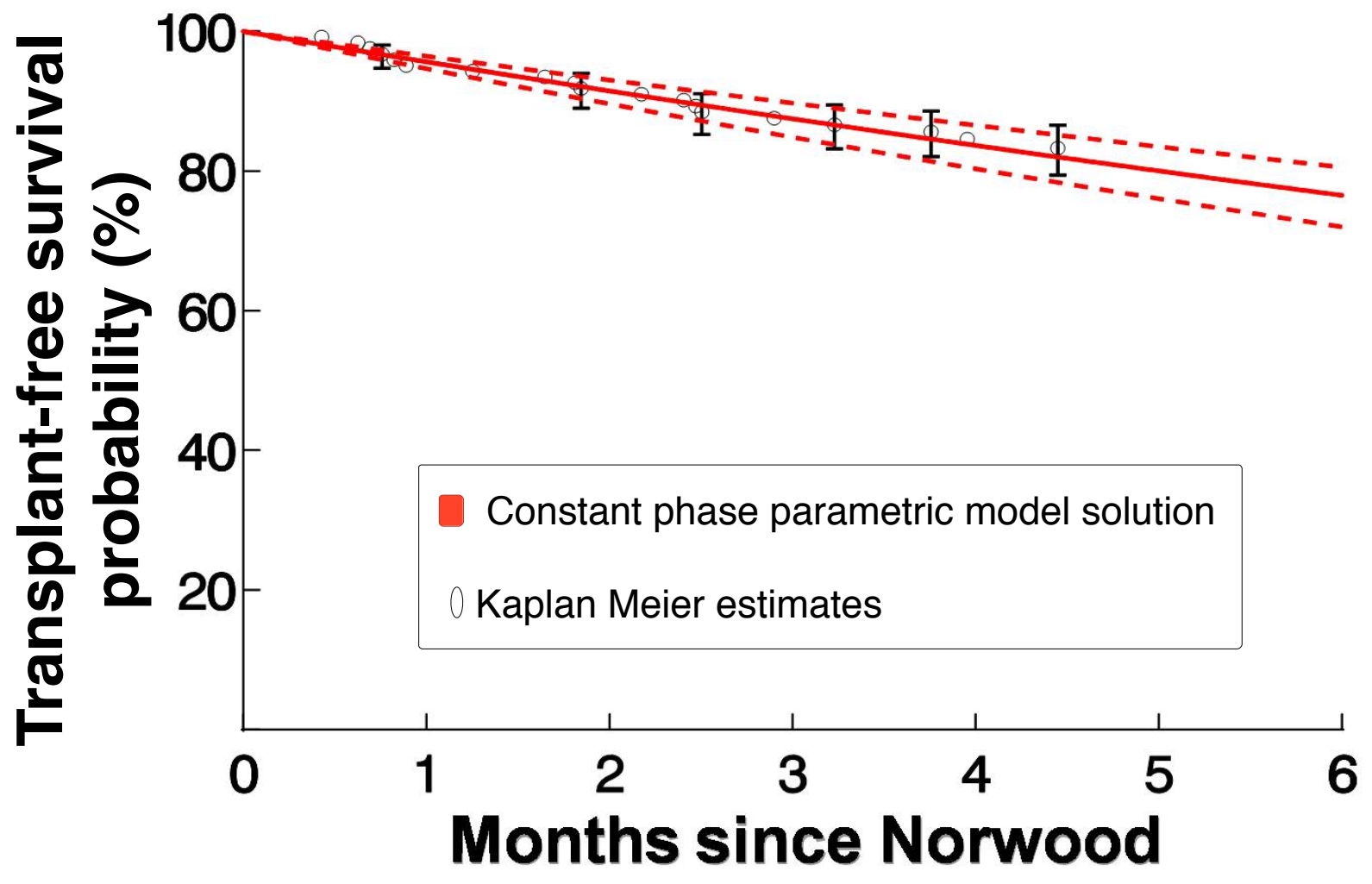

Figure 17. Goodness of fit of the constant phase parametric model. The nonparametric estimates were calculated and superimposed on the independent maximum likelihood estimates of the constant phase parametric model. The dashed line encloses the $68 \%$ confidence limits of the parametric estimates and the error bar represent the $95^{\text {th }}$ confidence interval of the Kaplan Meier estimates. 


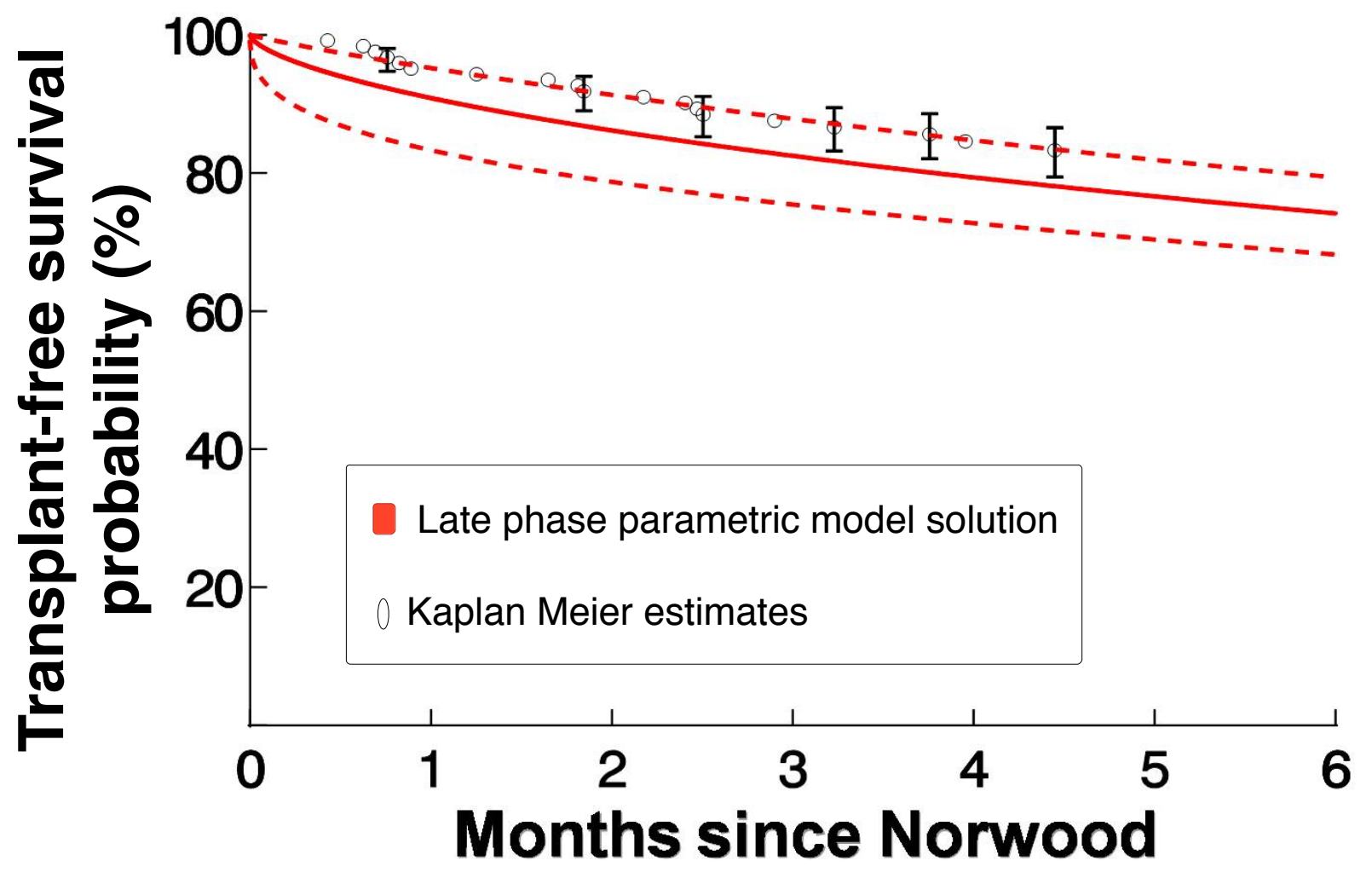

Figure 18. Goodness of fit of the late phase parametric model. The non-parametric estimates were calculated and superimposed on the independent maximum likelihood estimates of the late phase parametric model. The dashed line encloses the $68 \%$ confidence limits of the parametric estimates and the error bar represent the $95^{\text {th }}$ confidence interval of the Kaplan Meier estimates. 


\subsection{Risk factors associated with death or transplant in the ventricular dysfunction cohort}

\subsubsection{Variable selections}

We only performed variable selection for one hazard phase since our chosen parametric model is a single-phase hazard model. Bootstrapping with stepwise auto-selection identified eight possible variables with reliability above $25 \%$. No candidate variables were found to be interacting using the bootstrap bagging clustering algorithm, which assessed if variables are inter-related by evaluating how often two variables are found within the bootstrap samples. The risk factors retained after bagging (presence of post-Norwood infectious disease complications, surgeon performing 5 Norwood or less a year compare to more, presence of post-Norwood renal complications, larger LV end-diastolic volume, presence of neoaortic regurgitation on postNorwood echocardiogram, larger right ventricular end-diastolic long axis dimension, and worse right ventricle eccentricity) were then entered into the model and backward selection was performed using a $\mathrm{p}$ value retention threshold of 0.05 . The following variables were all significantly associated with death or transplant without stage II completion in our right ventricle dysfunction cohort: having a modified BT shunt compared to a RVPA conduit (parameter estimate (PE) 2.22, 95\% CI 0.19-4.24, p=0.03), having a surgeon with a Norwood procedure volume of five or less a year compared to more (PE 1.10, 95\% CI 0.24-1.97, p=0.01), experiencing an infectious disease complication post-Norwood procedure (PE 1.51, 95\% CI $0.62-2.40, \mathrm{p}<0.001$ ), and having a larger right ventricular end-diastolic long axis dimension measured on the post-Norwood echocardiogram (PE 1.06 per cm, 95\% CI 0.02-2.1, p=0.05) (Table 5). 


\subsubsection{Nature and influence of the risk factors}

\subsubsection{The hypothetical average patient predictions}

To explore the influence of the four identified risk factors in the final model, we solved the model parametric prediction plots using the formula of our parametric model. By using the average values of all four risk factors in the model equation solution, we were able to graph the transplant-free survival over time of the hypothetical average patient. We solved our model equation with the following mean values for the risk factors: shunt type $=0.6747967(1=\mathrm{BT}$ shunt), infectious disease complication $=0.4065041$ ( $1=$ presence of infectious disease complication), surgeon volume of 5 or less per year $=0.1869919$ ( $1=$ Five or less Norwood per year), right ventricular end diastolic dimension $=2.9782125 \mathrm{~cm}$ and obtained a six-month transplant-free survival of $87 \%$ (70\% CI 82 - 91\%) (Figure 19). Using this technique, we were then able to assess the influence of all three binary risk factors. For example, by specifying the value of all risk factors to the average values except for shunt type, we were able to graphically explore the effect of shunt type on the transplant-free survival for the hypothetical average patient (Figure 20). We demonstrated that a patient with BT shunt had a lower six-month survival than patients with an RVPA conduit (75\% (70\% CI 69 - $81 \%$ ) with BT shunt vs. $97 \%$ (70\% CI $92-99 \%$ with RVPA). Similarly, we created prediction plots of the hypothetical average patient looking at the influence of post-Norwood infectious disease complications and surgeon volume of five or less Norwood procedures per year compare to more volume (Figure $21 \& 22)$. These graphs demonstrated that the presence of infectious disease complications were associated with lower six-month transplant-free survival (71\% (70\% CI $61-80 \%)$ vs. $92 \%$ (70\% CI $89-96 \%$ ), Figure 21) and having a surgeon who performed five or less Norwood procedures a year compared to more was also associated with a worse six-month transplant-free survival ( $72 \%$ ( $70 \%$ CI $58-81 \%$ ) vs. $89 \%$ (70\% CI $85-93 \%)$, Figure 22$)$. We also wanted to improve our insight regarding the role that the right ventricle size was playing but as a continuous variable, a different technique was employed. We calculated the cumulative hazard at six months from the Norwood procedure for the hypothetical average patient using the mean values for all risk factors except for the right ventricular end-diastolic dimension and stratified the solution by shunt type. We chose six months for clinical reasons, since most children will undergo the stage II by four to six months of age. We then graphically represented the solution 
over the range of right ventricle sizes available within our population. The graph depicted the solution with survival at six-months post-Norwood procedure on the $\mathrm{Y}$ axis and the right ventricle size distribution on the $\mathrm{X}$ axis, demonstrating the higher risk of death or transplant that was associated with a larger right ventricle (Figure 23). The nomogram visually exploring the effect of right ventricle size on six month transplant-free survival also demonstrated that the right ventricle size seemed to have a more important influence on survival for patient with BT shunts than patients with an RVPA. We wanted to refine the model equation by adding an interaction term between right ventricular end-diastolic size and shunt type. The interaction term was not statistically significant ( $\mathrm{p}=0.76)$, demonstrating that there was no significant interaction between the right ventricle size and shunt type. As a result, our final model remained unchanged with the same four independent risk factors for death or heart transplant without stage II completion.

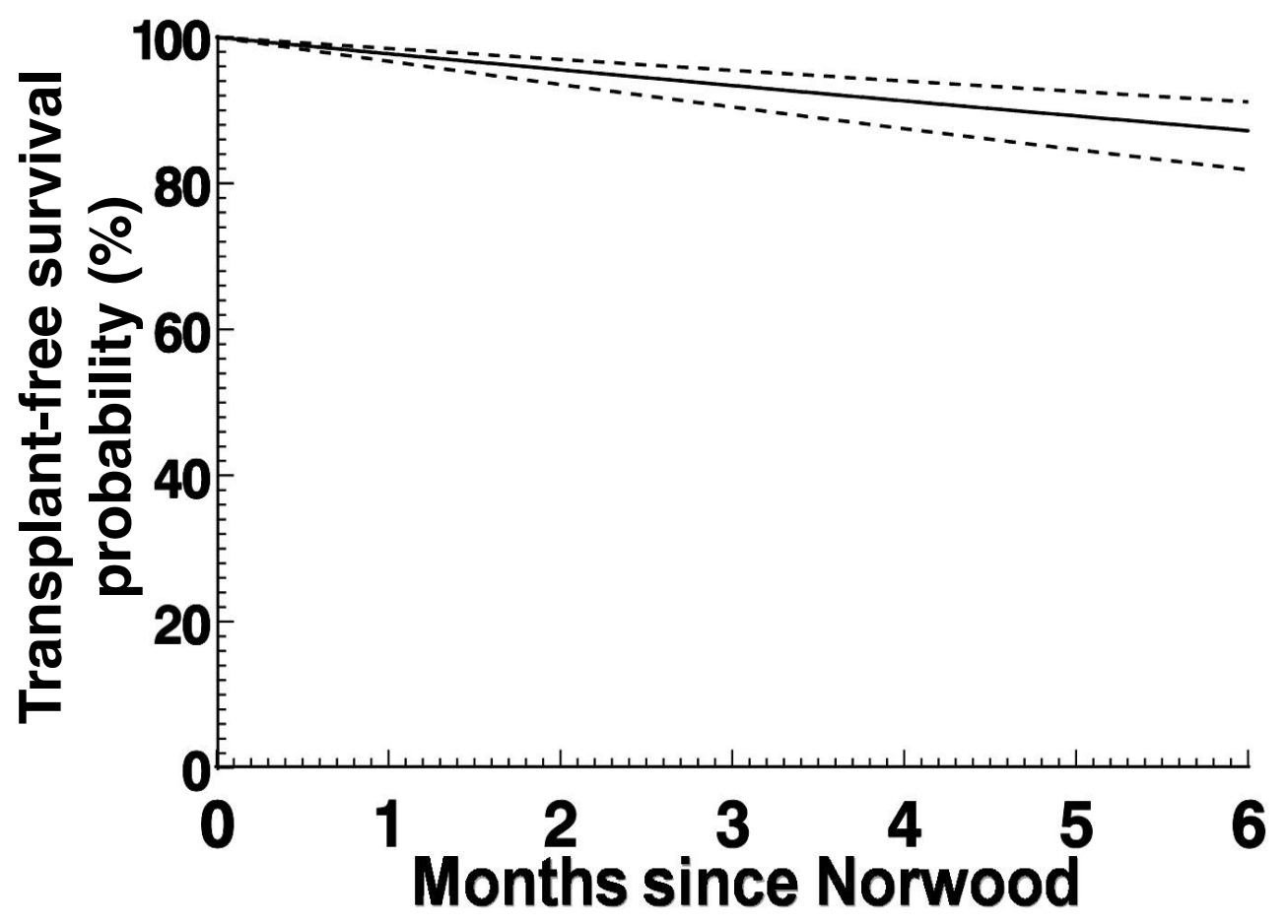

Figure 19. Transplant-free survival over time in months for a hypothetical average patient with all the risk factor values set as means. We solved our model equation with the following mean values for the risk factors: shunt type $=0.6747967$ ( $1=\mathrm{BT}$ shunt), infectious disease complication $=0.4065041$ ( $1=$-presence of infectious disease complication), surgeon 
volume of 5 or less per year $=0.1869919(1=$ Five or less Norwood per year $)$, right ventricular end diastolic dimension $=2.9782125 \mathrm{~cm}$. The average predicted 6 months transplant-free survival was $87 \%(70 \%$ CI $92-91 \%)$.

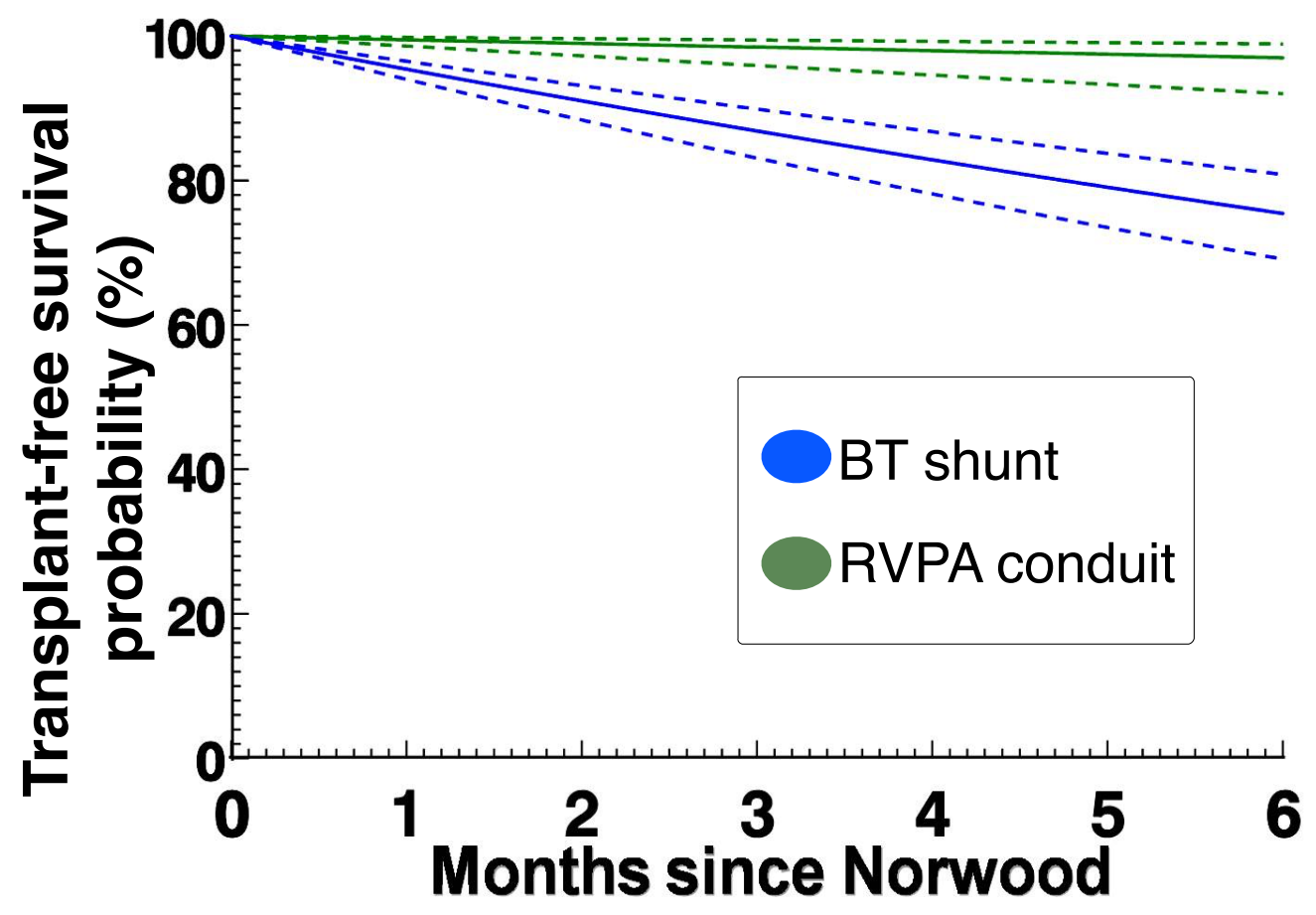

Figure 20. Transplant-free survival over time in months for a hypothetical average patient with all risk factor values set at means stratified by shunt type. We solved our model equation with the following mean values for the risk factors: infectious disease complication $=0.4065041$ ( 1 =presence of infectious disease complication), surgeon volume of 5 or less per year $=0.1869919(1=$ Five or less Norwood per year $)$, right ventricular end diastolic dimension $=2.9782125 \mathrm{~cm}$. The average predicted transplant-free survival at 6 months was $75 \%$ (70\% CI $69-81 \%)$ with BT shunt vs. $97 \%(70 \%$ CI $92-99 \%)$ with RVPA. 


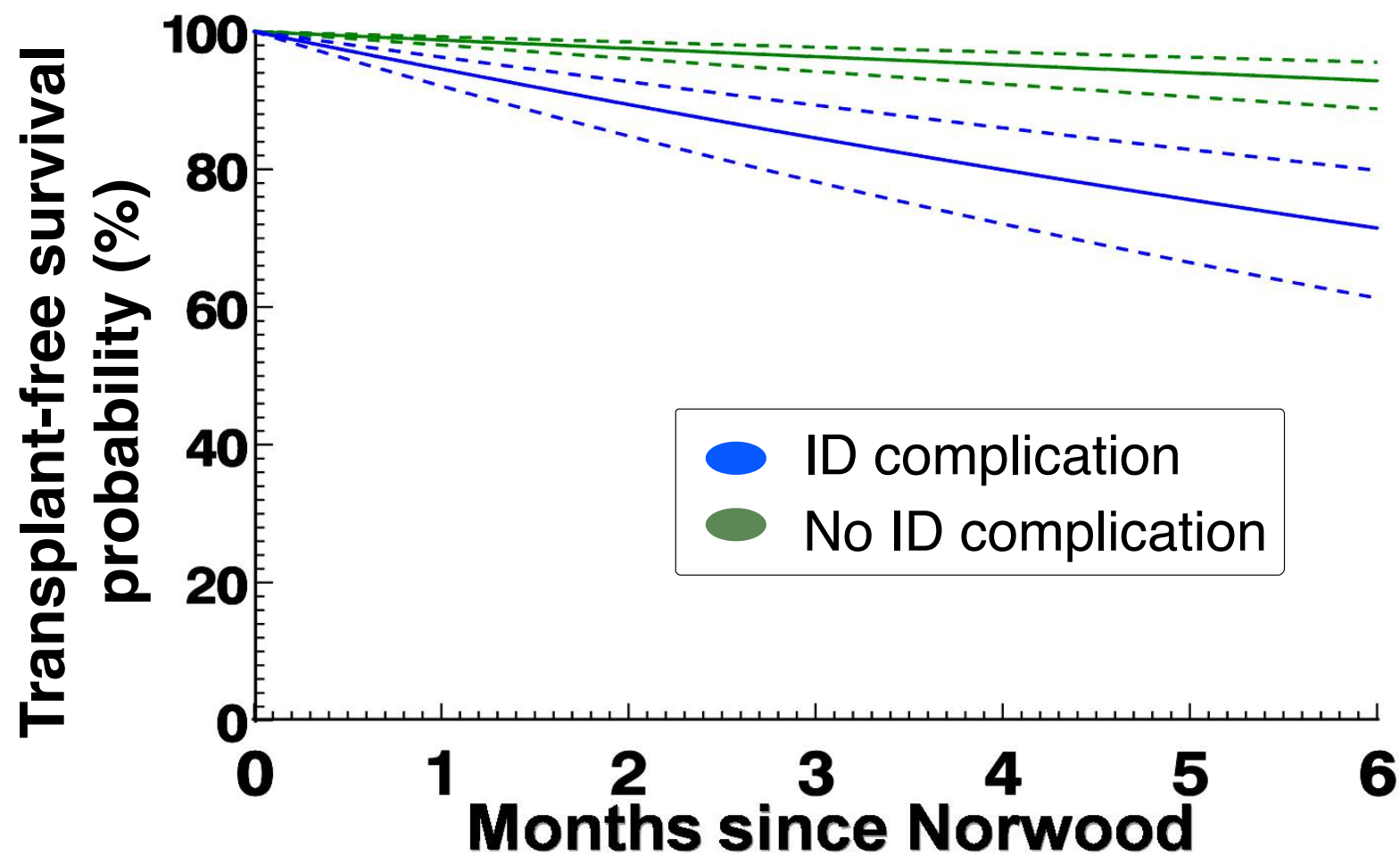

Figure 21. Transplant-free survival over time in months for a hypothetical average patient with all risk factor values set at means stratified by the presence of infectious disease complication post-Norwood procedure. We solved our model equation with the following mean values for the risk factors: shunt type $=0.6747967 \quad(1=\mathrm{BT}$ shunt), surgeon volume of 5 or less per year $=0.1869919$ ( $1=$ Five or less Norwood per year), right ventricular end diastolic dimension $=2.9782125 \mathrm{~cm}$. The average predicted transplant-free survival at 6 months was $71 \%(70 \%$ CI $61-80 \%)$ with an post-Norwood infectious disease complication vs. $92 \%(70 \%$ CI $89-96 \%)$ without. 


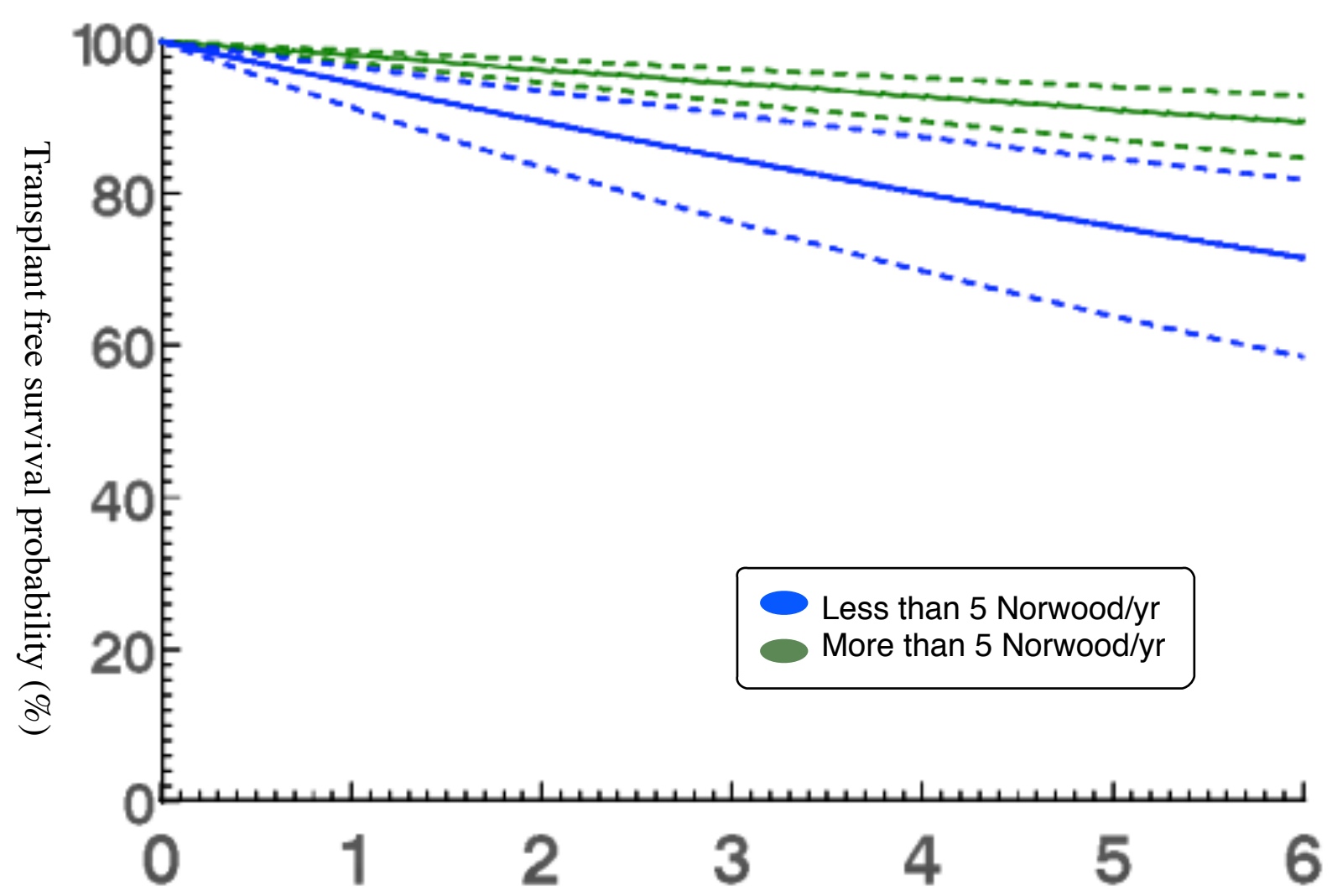

Time from Norwood procedure (months)

Figure 22. Transplant-free survival overtime in months for a hypothetical average patient with all the risk factor values set as means stratified by surgeon volume with less or equal to five Norwood procedures per year compared to more than five. We solved our model equation with the following mean values for the risk factors: shunt type $=0.6747967$ ( $1=\mathrm{BT}$ shunt $)$, infectious disease complication $=0.4065041$ ( $1=$ presence of infectious disease complication), right ventricular end diastolic dimension $=2.9782125 \mathrm{~cm}$. The average predicted transplant-free survival at 6 months was $72 \%(70 \%$ CI $58-81 \%)$ with a surgeon volume of five or less Norwood procedure a year vs. $89 \%$ (70\% CI $85-93 \%)$ with higher volume. 


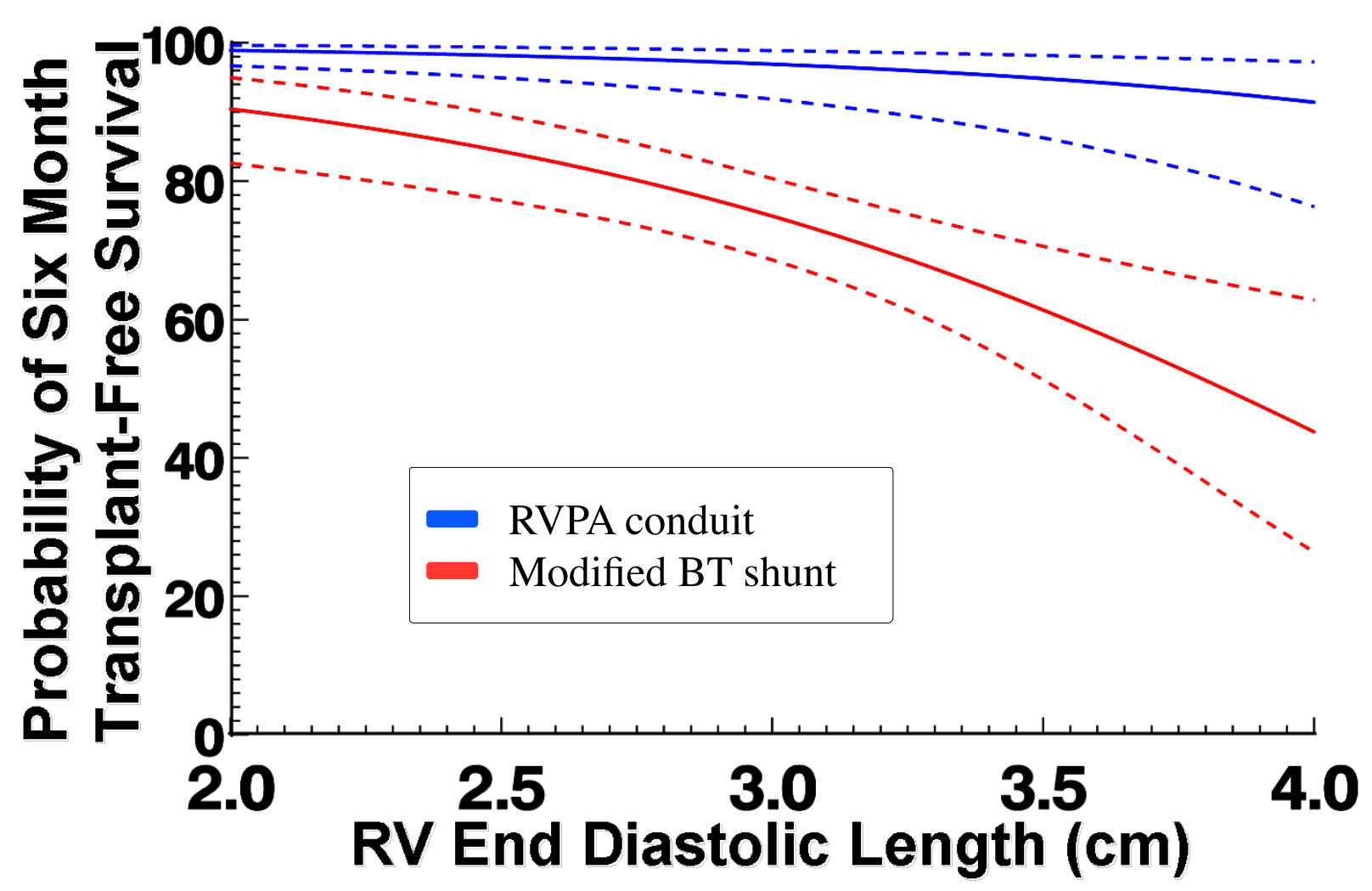

Figure 23. Nomogram representing the influence of post-Norwood right ventricular end-diastolic dimension on transplant-free survival at six months post-Norwood procedure for the hypothetical average patient stratified by shunt type. We solved our model equation with the following mean values for the risk factors: infectious disease complication $=0.4065041$ ( $1=$ presence of infectious disease complication $)$ and surgeon volume of 5 or less per year $=0.1869919$ ( $1=$ Five or less Norwood per year).

\subsubsection{The cohort average predictions}

The hypothetical average patient might not be representative of our study cohort. For example, we used mean values for binary risk factors, which has no clinical meaning. Therefore, we decided to generate time-related survival curves for each patient in our ventricular dysfunction cohort. We averaged the 123 patient-specific cumulative hazards at each time points. By doing this, we were able to plot the average transplant-free survival for the ventricular dysfunction 
cohort patients (Figure 24) demonstrating a predicted six-month transplant-free survival of $77 \%$ (70\% CI $69-83 \%$ ). Using the cohort of patients, we could again graphically represented the influence of all the three identified binary risk factors by averaging subgroups of patients with or without a specific risk factor and comparing them visually (Figure 25, 26 \& 27). This demonstrated a lower 6 months transplant-free survival associated with BT shunt compared to RVPA conduit (67\% (70\% CI 58 - $75 \%$ ) for BT shunt vs. $97 \%$ (70\% CI $92-99 \%$ ) for RVPA, Figure 25), with the presence of infectious disease complications (61 \% (70\% CI $58-70 \%$ ) with infectious complication post Norwood procedure vs. $88 \%$ (70\% CI $82-92 \%)$ without, Figure 26) and with a surgeon who performed five or less Norwood procedures a year compare to more (54 \% (70\% CI $44-65 \%$ ) with five or less Norwood procedures per year vs. $82 \%$ (70\% CI 75 $88 \%$ ) with more, Figure 27). Similar to the analysis performed for the hypothetical average patient, we wanted to improve our insight regarding the role that the right ventricle size was playing but as a continuous variable, a different technique was employed. We calculated the cumulative hazard at six months from the Norwood procedure for all the patients within our dysfunction cohort using all their real values for all risk factors except for the right ventricular end-diastolic dimension. We chose six months for clinical reasons, since most children will undergo the stage II by four to six months of age. We then graphically represented the solution over the range of right ventricle sizes of our study cohort. and stratified the solution by shunt type. The graph depicted the solution with survival at six-months post-Norwood procedure on the $\mathrm{Y}$ axis and the right ventricle size distribution on the $\mathrm{X}$ axis, demonstrating the higher risk of death or transplant that was associated with a larger right ventricle (Figure 28). 


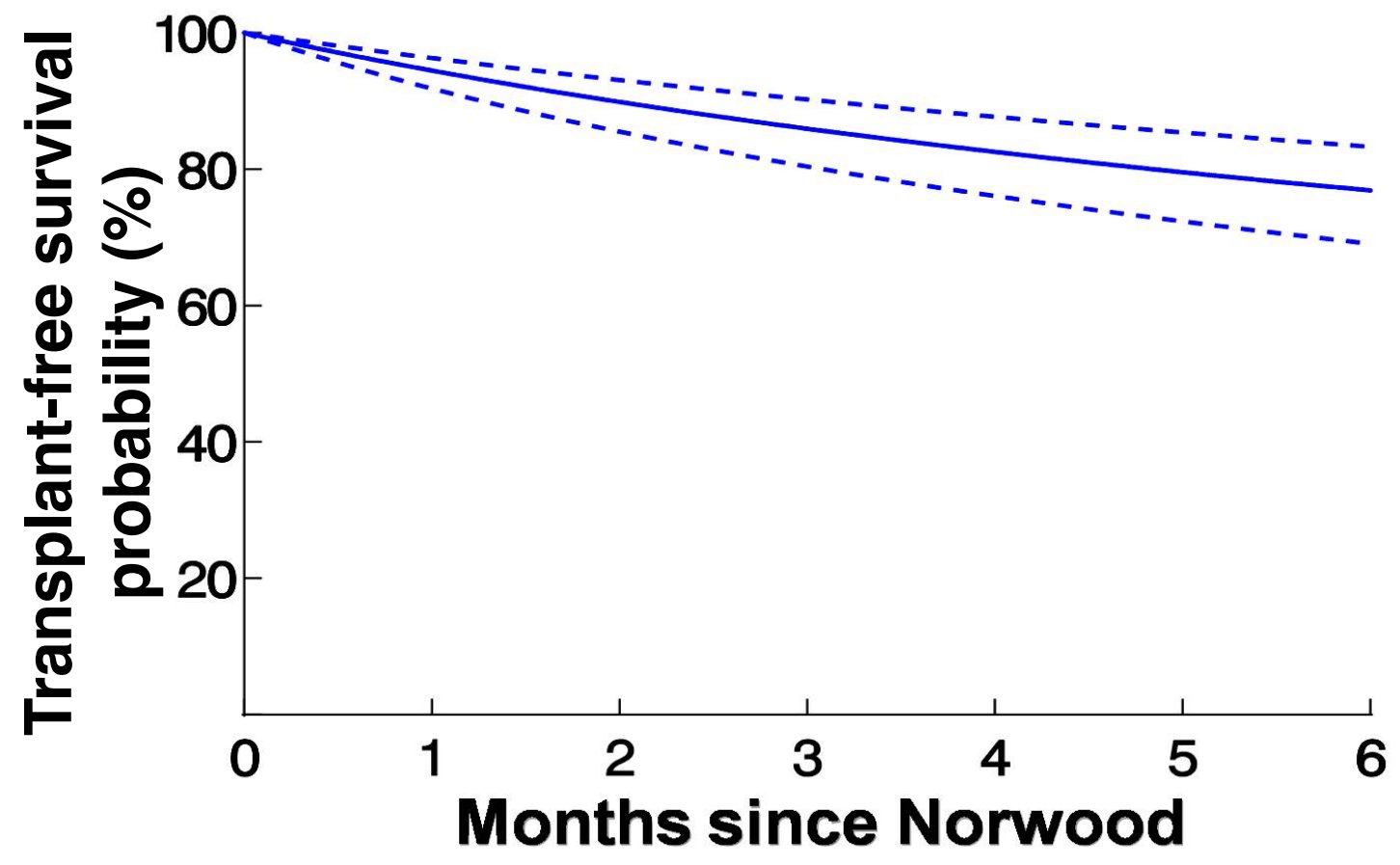

Figure 24. Average parametric transplant-free survival for the ventricular dysfunction cohort patients. We averaged the 123 patient-specific cumulative hazards at each time points. The average predicted transplant-free survival at 6 months was $77 \%$ ( $70 \% \mathrm{CI}$ $69-83 \%)$. 


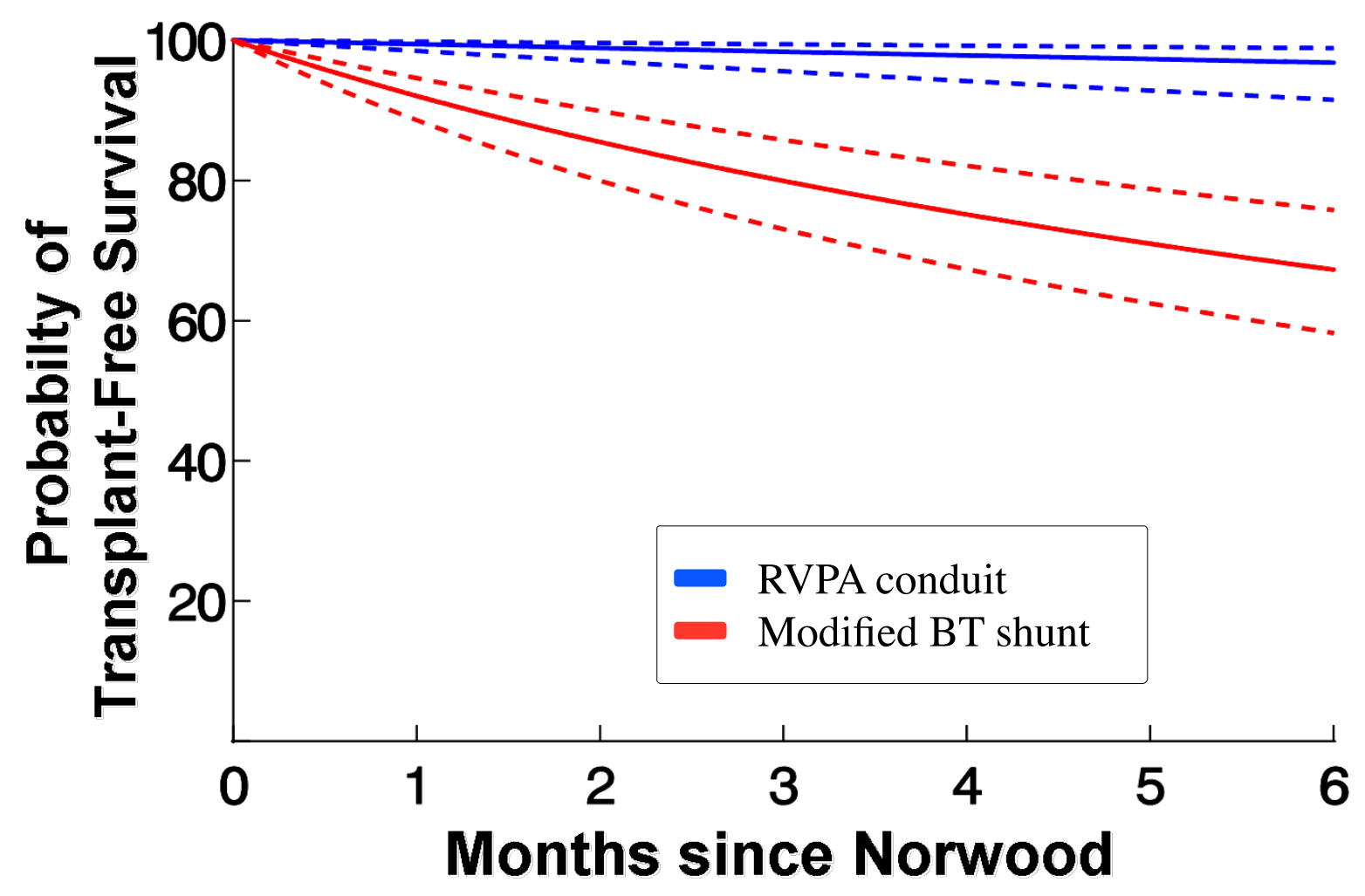

Figure 25. Average parametric transplant-free survival for the ventricular dysfunction cohort patients stratified by shunt type. We averaged the 123 patientspecific cumulative hazards at each time points and averaged the subgroups of patients with a BT shunt and with an RVPA. The average predicted transplant-free survival at 6 months was $67 \%$ (70\% CI $58-75 \%)$ for BT shunt vs. $97 \%(70 \%$ CI $92-99 \%)$ for RVPA 


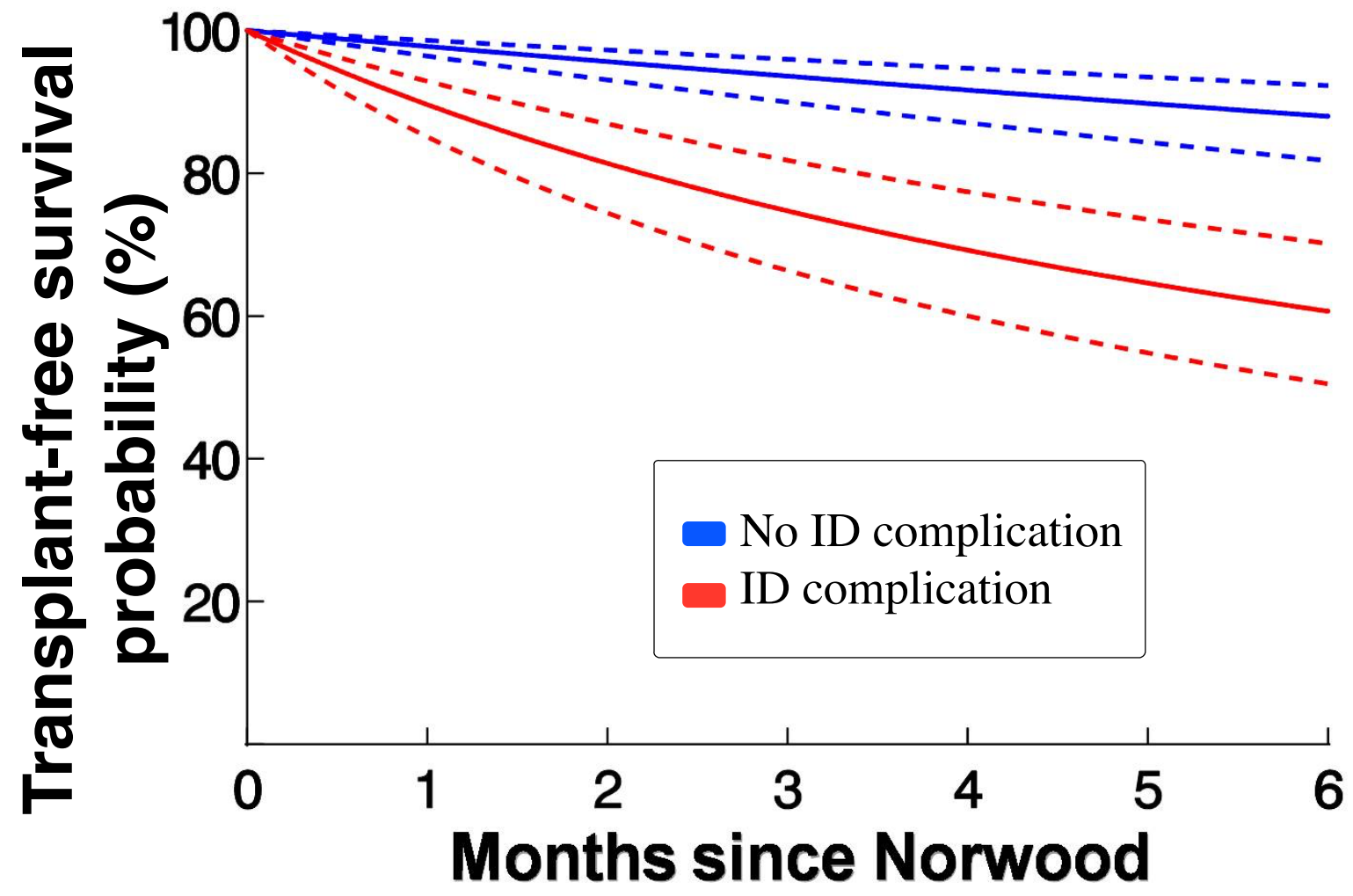

Figure 26. Average parametric transplant-free survival for the ventricular dysfunction cohort patients stratified by the presence or absence of infectious disease complications post-Norwood procedure. We averaged the 123 patient-specific cumulative hazards at each time points and averaged the subgroups of patients with or without an infectious disease complication in the post-Norwood period. The average predicted transplantfree survival at 6 months was $61 \%(70 \%$ CI $58-70 \%)$ with infectious complication post Norwood procedure vs. $88 \%$ (70\% CI $82-92 \%)$ without. 


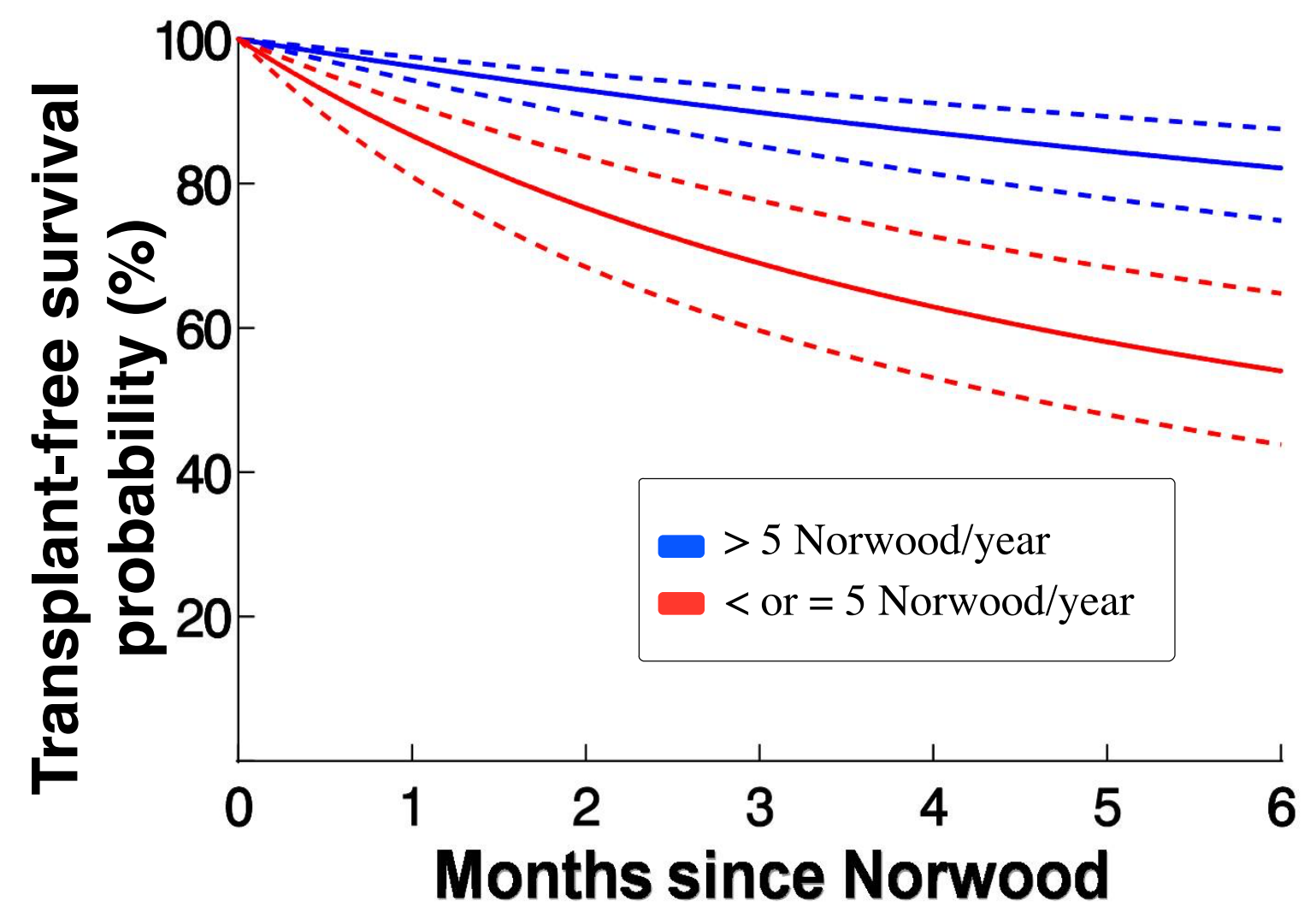

Figure 27. Average parametric transplant-free survival for the ventricular dysfunction cohort patients stratified by surgeon volume with less or equal to 5 Norwood procedures per year compare to more than five. We averaged the 123 patient-specific cumulative hazards at each time points and averaged the subgroups of patients with or without a surgeon that performed 5 or less Norwood procedures per year. The average predicted transplant-free survival at 6 months was $54 \%(70 \%$ CI $44-65 \%)$ with a surgeon volume of five or less Norwood procedure a year vs. $82 \%(70 \%$ CI $75-88 \%)$ with higher volume. 


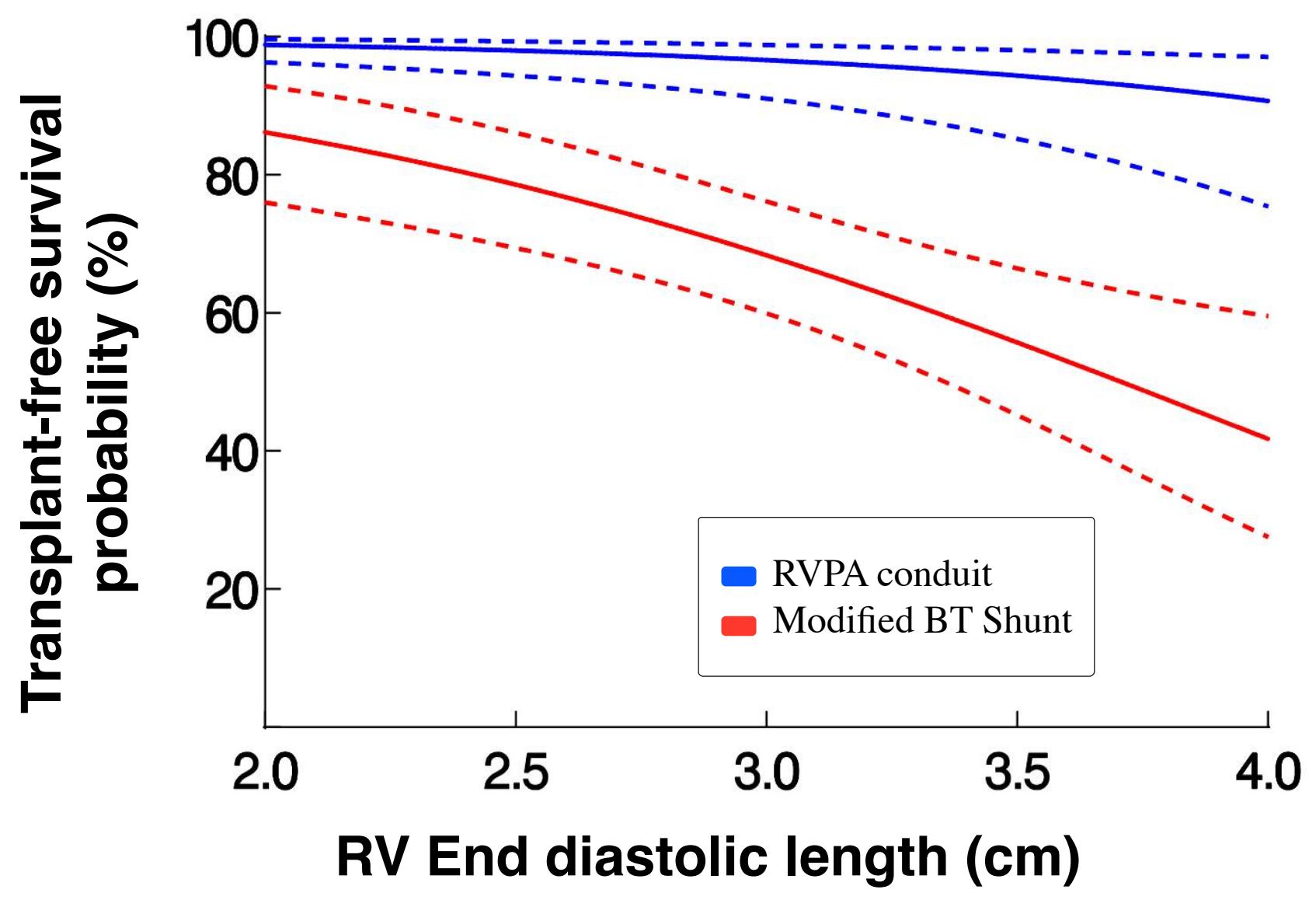

Figure 28. Nomogram representing the influence of post-Norwood right ventricular end-diastolic dimension on transplant-free survival at six months post-Norwood procedure for ventricular dysfunction cohort patients stratified by shunt type. We calculated the cumulative hazard at six months from the Norwood procedure for all the patients within our dysfunction cohort using all their real values for all risk factors then graphically represented the solution over the range of right ventricle sizes of our study cohort. stratified by shun type

\subsubsection{Final model}

Our final model remained unchanged with the same four independent risk factors for death or heart transplant without stage II completion. The independent risk factors of our final model 
were having a modified BT shunt compared to a RVPA conduit (PE 2.22, 95\% CI 0.19-4.24, $\mathrm{p}=0.03$ ), experiencing an infectious disease complication post-Norwood procedure (PE 1.51, 95\% CI 0.62-2.40, $\mathrm{p}<0.001$ ), having a surgeon Norwood procedure volume of five or less a year compare to more (PE 1.10, 95\% CI 0.24-1.97, p=0.01), and having a larger RV end-diastolic long axis dimension measured on the post-Norwood echocardiogram (PE 1.06, 95\% CI 0.02-2.1, $\mathrm{p}=0.05)$ (Table 5).

\subsubsection{Best and worst-case scenario predictions}

In order to help the clinicians make appropriate decisions for their patients, we used our model equation to predict survival for the best-case and worst-case scenarios in terms of patients characteristics. We created two model solutions where one used the best values for the identified risk factors and the other used the worse values for the identified risk factors. In other words, patient with and without the final model risk factors. For the best-case scenario, we solved the model equation with having a RVPA conduit, having no infectious disease complication postNorwood, having a surgeon that performed more than 5 Norwood procedures per year and having a right ventricular end-diastolic dimension of $2.01 \mathrm{~cm}$, the minimal value for the right ventricular dysfunction cohort. The worst-case scenario, we solved the model equation with having a BT shunt, having an infectious disease complication in the post-Norwood period, having a surgeon who performed 5 or less Norwood per year and having a right ventricle enddiastolic dimension of $3.99 \mathrm{~cm}$, the maximal value for the right ventricular dysfunction cohort. We obtained predicted survivals (Figure 29) demonstrating that for patients with all the risk factors the predicted transplant-free survival at six months included zero. Actually, the predicted 6 months transplant free survival for the worst-case scenario was $1 \%$ (70\% CI 0-13\%) compared to $99 \%$ (70 \% CI 98-100\%) for the best-case scenario. We then stratified the two scenarios by shunt type to see the effect of risk factors on patients with the different shunt types (Figure 30). We predicted a 6 months transplant-free survival of $1 \%(70 \% \mathrm{CI} 0-13 \%)$ for the BT shunt group worst-case scenario compared to $96 \%$ (70\% CI 91-98\%) for the BT shunt group bestcase scenario. We also predicted a 6 months transplant-free survival of $59 \%$ (70\% CI 23-87 \%) for the RVPA conduit group worst-case scenario versus $99 \%$ (70\% CI 23-87\%) for the RVPA

conduit group best-case scenario. Similarly, we wanted to visually assess the effect of the right 
ventricle size on the same two scenarios stratified by shunt type (Figure 31), again demonstrating visually the cumulative effect of the different risk factors.

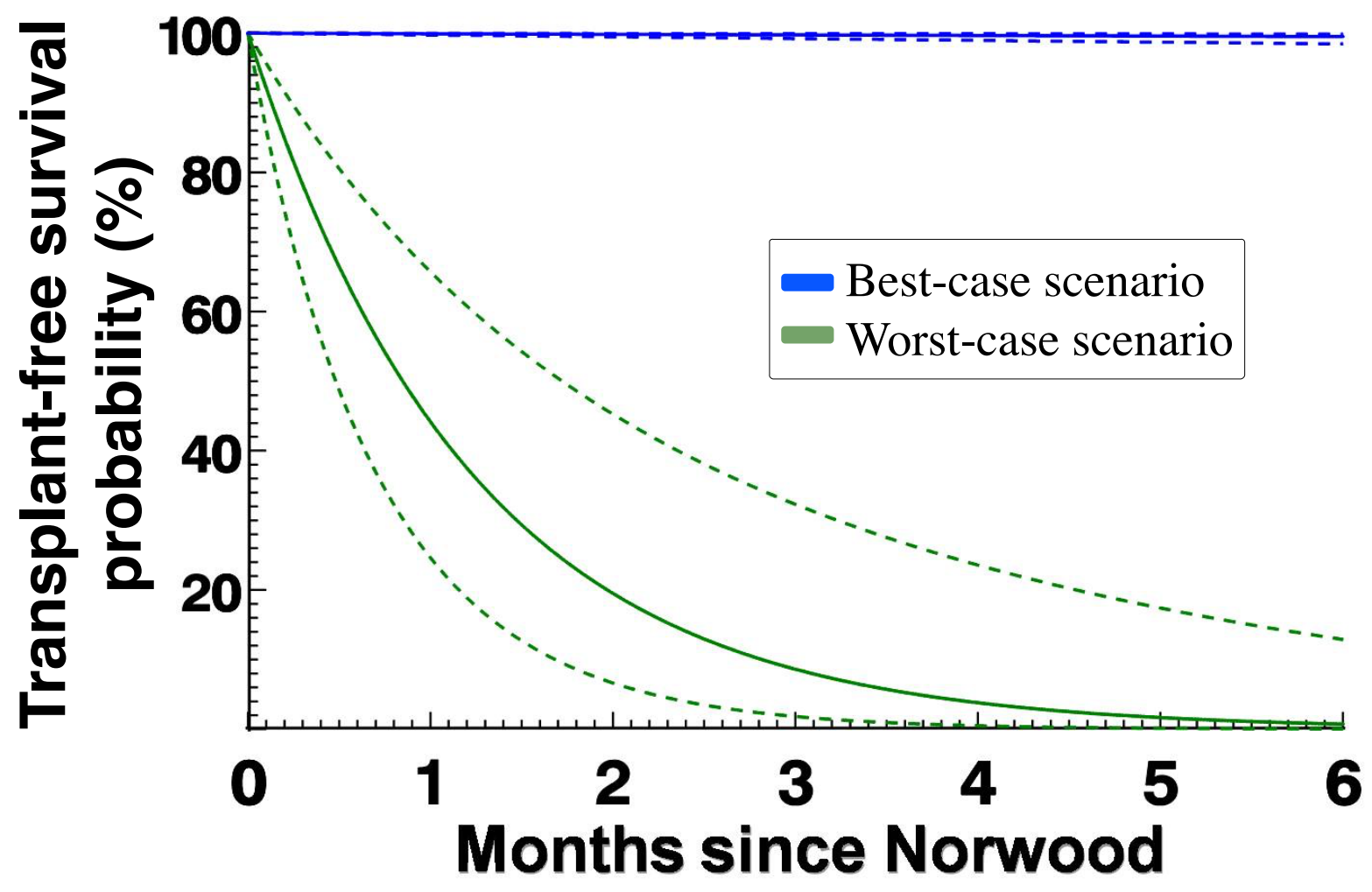

Figure 29. Best-case and worst-case scenarios predicted survival. For the best-case scenario, we solved the model equation with having a RVPA conduit, having no infectious disease complication post-Norwood, having a surgeon that performed more than 5 Norwood procedures per year and having a right ventricular end-diastolic dimension of $2.01 \mathrm{~cm}$, the minimal value for the right ventricular dysfunction cohort. The worst-case scenario, we solved the model with having a BT shunt, having an infectious disease complication in the postNorwood period, having a surgeon who performed 5 or less Norwood per year and having a right ventricle end-diastolic dimension of $3.99 \mathrm{~cm}$, the maximal value for the right ventricular dysfunction cohort. The predicted 6 months transplant free survival for the worst-case scenario was $1 \%(70 \%$ CI 0-13\%) compared to $99 \%$ (70\% CI 98-100\%) for the best-case scenario. 

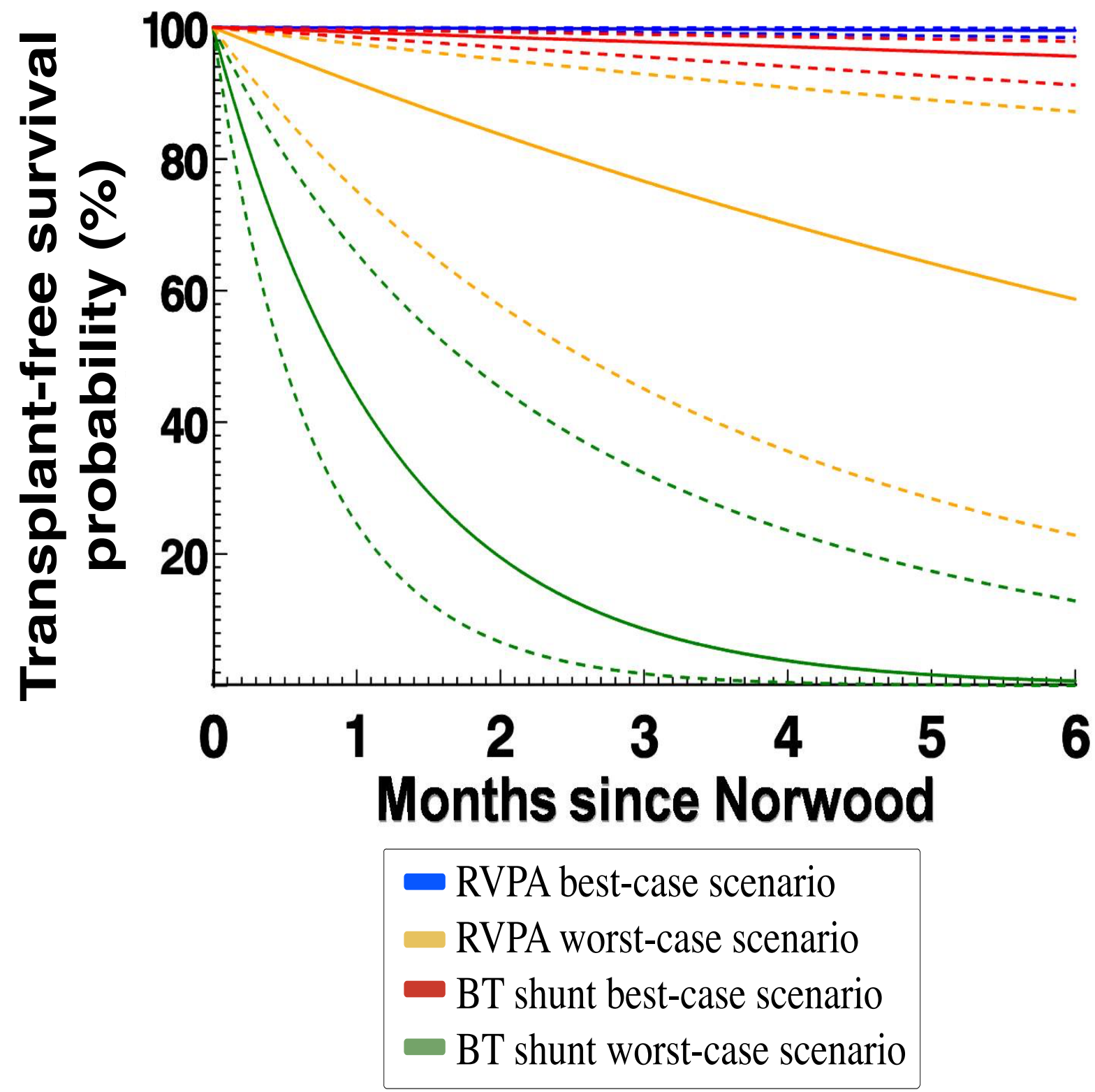

Figure 30. Best-case and worst-case scenarios predicted survival stratified by shunt type. For the best-case scenarios, we solved the model equation with having no infectious disease complication post-Norwood, having a surgeon that performed more than 5 Norwood procedures per year and having a right ventricular end-diastolic dimension of $2.01 \mathrm{~cm}$, the minimal value for the right ventricular dysfunction cohort and we then stratified the solution by shunt type. For the worst-case scenarios, we solved the model with having an infectious disease complication in the post-Norwood period, having a surgeon who performed 5 or less Norwood per year and having a right ventricle end-diastolic dimension of $3.99 \mathrm{~cm}$, the maximal value for the right ventricular dysfunction cohort and we then stratified the solution by shunt type. We 
predicted a 6 months transplant-free survival of $1 \%(70 \%$ CI 0-13\%) for the BT shunt group worst-case scenario compared to $96 \%$ ( $70 \%$ CI 91-98 \%) for the BT shunt group best-case scenario. We also predicted a 6 months transplant-free survival of $59 \%$ (70\% CI $23-87 \%)$ for the RVPA conduit group worst-case scenario versus $99 \%$ (70\% CI 23-87\%) for the RVPA conduit group best-case scenario. 


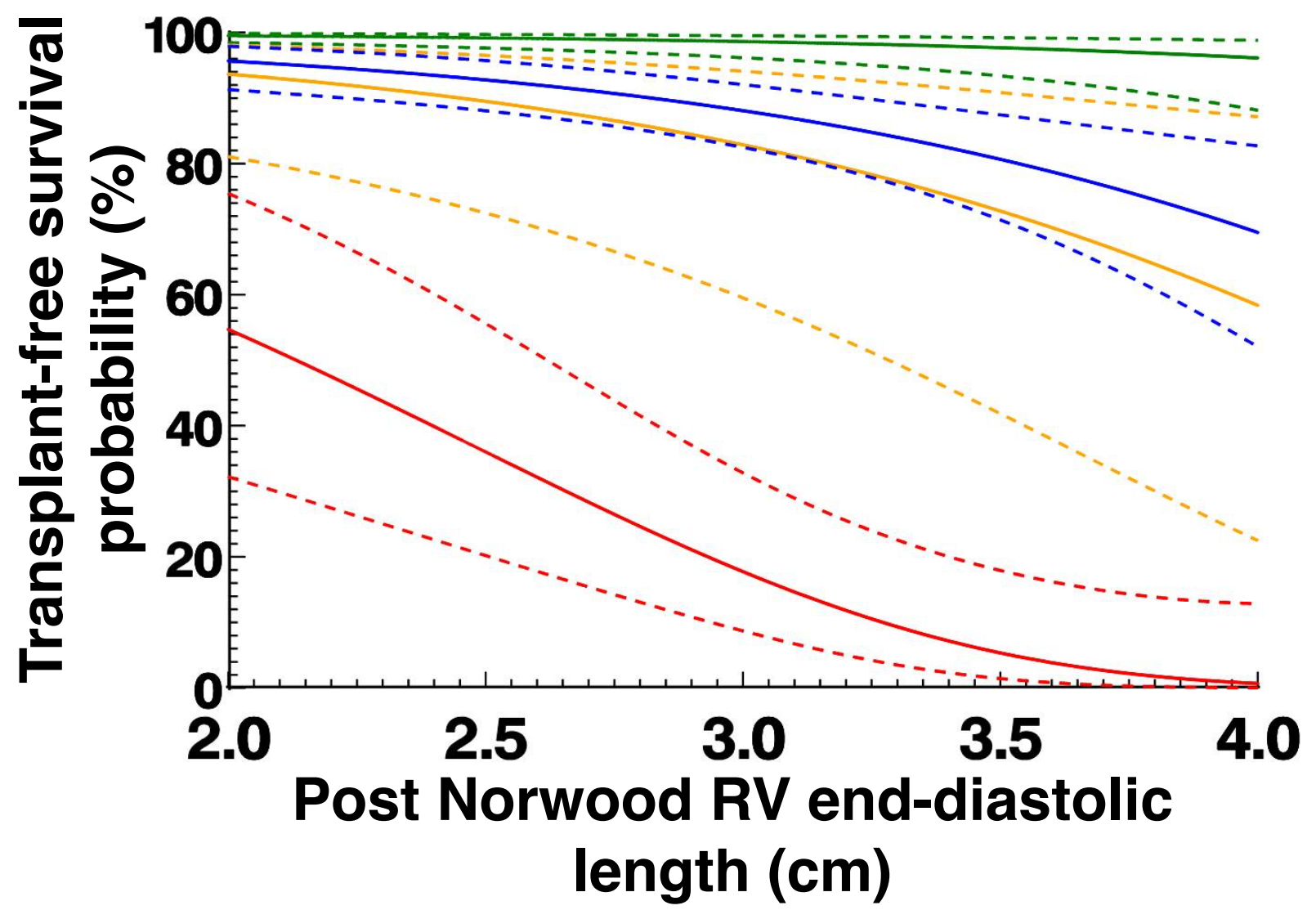

\begin{tabular}{|l|} 
RVPA best-case scenario \\
RVPA worst-case scenario \\
BT shunt best-case scenario \\
BT shunt worst-case scenario
\end{tabular}

Figure 31. Nomogram representing the association of post-Norwood right ventricular end-diastolic dimension with transplant-free survival at 6 months postNorwood procedure for the best-case and worst-case scenarios stratified by shunt type. We calculated the 6 months cumulative hazard for the best and worst scenario stratified by shunt type and presented them over the right ventricular end-diastolic size on the y axis. For the best-case scenarios, we solved the model equation with having no infectious disease complication post-Norwood, having a surgeon that performed more than 5 Norwood procedures 
per year. For the worst-case scenarios, we solved the model equation with having an infectious disease complication in the post-Norwood period and having a surgeon who performed 5 or less Norwood.

\subsection{Assumptions and model verifications}

For our model, the assumption of random censoring was respected because the data were sourced from a randomized clinical trial and important effort was taken to keep patients within the trial, which resulted in all patients receiving follow-up within the dysfunction cohort. In addition, each variable was tested for outliers and missing data; therefore, we can assume the absence of influential observations. Absence of influential outliers was also assessed using casewise diagnostics and, based on visual inspection of DFBETAS, CookD, and studentized residuals, no outliers were deemed significant (Figure 31). Model over specification was assessed using warning of lack of convergence of the model or inappropriate $95 \%$ confidence intervals for parameter estimates. Finally, we assessed the goodness of fit of our multivariable parametric model. To do this, we solved the equation for each patients within the cohort using their specific values for the identified risk factors. We then averaged all the patients predicted survival curves together with the $70 \%$ confidence interval. Then we superimposed the Kaplan-Meier estimates and their $95 \%$ confidence intervals to the graph. Similar to the initial goodness of fit testing, since the Kaplan-Meier estimates and their associated confidence intervals were within the confidence of our parametric estimates, the model was considered adequate (Figure 32). There was no residual risk left unaccounted by our parametric model (Sergeant, Blackstone \& Meyns 1997). We also verified that at the six months time interval of interest, we had more than $10 \%$ of the cohort still at risk. At 200 days from randomization, there were 22 patients still remaining at risk, representing $18 \%$ of the dysfunction cohort. Overall, we felt that our model was valid for this time interval and confirm our decision to represent our graphs to 6 months post randomization. 


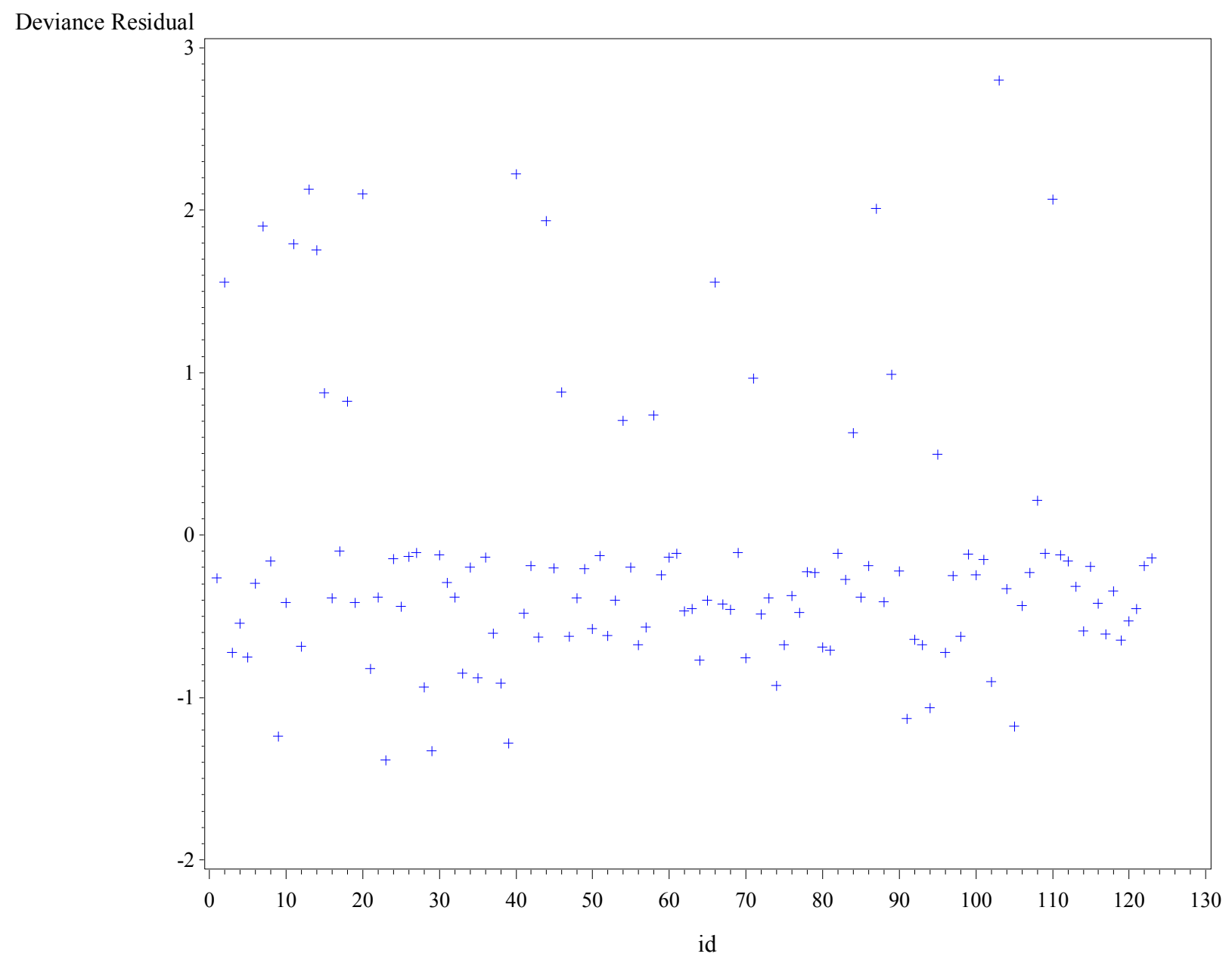

Figure 32. Deviance residual plotted by unique identifier. Deviance residual plotted by unique identifier demonstrating that most values are just below the zero value for deviance residual confirming the absence of influential outliers. 


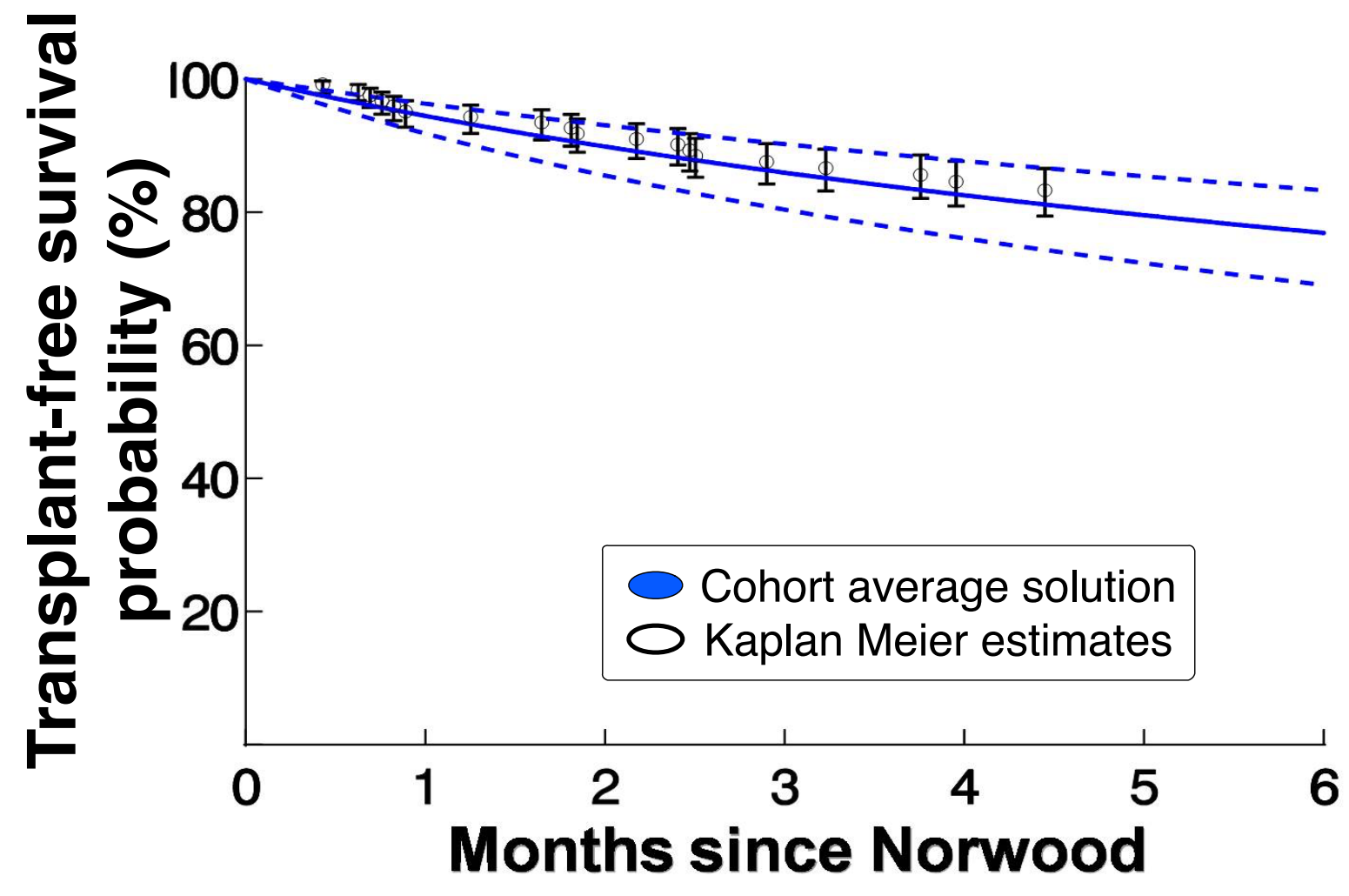

Figure 33. Goodness of fit of the final multivariable parametric model. Solution of the multivariable parametric model for the cohort patients with superimposed Kaplan-Meier estimates and their 95\% confidence intervals. The non-parametric estimates were calculated and superimposed on the average predicted survival for all patients within the cohort based on the final multivariable parametric model. The dashed line encloses the $68 \%$ confidence limits of the parametric estimates. 


\section{Chapter 5}

\section{Discussion}

With this analysis, we sought to determine both patient and procedural factors that could help

predict outcomes in a cohort of HLHS patients with right ventricle dysfunction post-Norwood procedure. We were able to evaluate the hazard for death or transplant post-Norwood procedure using multivariable parametric modeling techniques. We identified that having a modified BT shunt, an infectious disease complication post-Norwood procedure, a surgeon with volume of five or less Norwood procedures a year and a larger right ventricle post-Norwood procedure, were all independently associated with death or transplant post-Norwood without stage II completion in this cohort. This demonstrates that clinical information can assist the clinician to identify this at risk population and facilitate clinical decision making, such as early transplant listing.

\subsection{Modified BT shunt versus RVPA conduit}

\subsubsection{Effect of shunt type on survival in the SVR trial analyses}

One of the important finding of this study is the strong association of shunt type on survival in this at risk population. Having a BT shunt and ventricular dysfunction by themselves would not be sufficient for considering early transplant listing, however, with the addition of other risk factors, the predicted survival became dismal (Figure 30). Shunt type is a risk factor for mortality in patients with HLHS previously reported in the SVR trial analyses. The primary outcome of the SVR trial was death or heart transplantation at 12 months post randomization (Ohye et al. 2010). 
This trial showed that there were significantly fewer deaths or heart transplants in the RVPA group compared to the BT shunt group at 12 months post randomization (26\% vs. $36 \%$, $\mathrm{p}=0.01$ ). Shunt type was also found to be associated with interstage death in an univariate analysis (OR 3.44, p<0.001), but only of those with mild or no AVVR in the multivariable analysis (OR 9.7, $\mathrm{p}<0.001)$ (Ghanayem et al. 2012). RVPA conduit was no longer associated with superior transplant-free survival at three years post-randomization (Newburger et al. 2014). However, a recent analysis of an inception cohorts of neonates with critical left ventricular outflow tract obstruction, showed that RVPA was associated with the greater overall survival at 4 years follow-up (RVPA 76 \% vs. Hybrid $61 \%$ vs. modified BT 60 \%, p<0.001) (Wilder et al. 2016).

\subsubsection{Effect of shunt type on right ventricular function in the SVR trial}

In the main SVR trial analysis, RVEF was significantly higher in the RVPA conduit group compared to the BT shunt group at the post-Norwood echocardiogram ( $49 \%+/-7 \%$ for RVPA vs. $44 \%+/-8 \%$ for BT shunt, $\mathrm{p}<0.001)$ but not significantly different at the Stage II and 14 months follow-up echocardiograms. Similar differences were shown for the RVFAC measurements $(38 \%+/-7 \%$ for RVPA vs. $35 \%+/-8 \%$ for BT shunt at the post-Norwood echocardiogram, $\mathrm{p}<0.001)$. Despite this significant difference in right ventricular function at the post-Norwood echocardiogram, right ventricular function was not an independent risk factor for death for the post-Norwood hospitalization period, for the 30-days post-Norwood period (Tabutt et al. 2012) and for the interstage period (Guanayem et al. 2014). However, shunt type was a significant independent risk factor at 12 months post- randomization (26\% for RVPA vs. $36 \%$, for BT shunt, $\mathrm{p}=0.01$ ). This is demonstrating potentially that the effect of shunt type on survival to stage II in our ventricular dysfunction is unique to this high-risk population with ventricular dysfunction. 


\subsubsection{Effect of shunt type in our right ventricular dysfunction cohort}

Within our cohort of patients with right ventricular dysfunction, the stark difference in survival between shunt types with ventricular dysfunction (67\% (70 \% CI 58 - $75 \%$ ) for BT shunt vs. 97 $\%(70 \%$ CI 92 - 99\%) for RVPA, Figure 22)) and the strong effect of shunt type on risk of death

or heart transplant (PE 2.22, 95\% CI 0.19-4.24, $\mathrm{p}=0.03$, table 5) is remarkable. In addition, the demonstration of the cumulative effect of the other risk factors on transplant-free survival in addition to shunt (Figure 30) suggests that the physiology of patients with a BT shunt and RVPA conduit is fragile. The hypothesis that poor coronary perfusion associated with the diastolic run off caused by a BT shunt which leads to a poor myocardia perfusion (Figure 34) (Bradley et al. 2004; Ghanayem et al. 2006; Donnelly et al. 1998; DeCampli et al. 2013; Hsia et al. 2011), is reinforced by our results, since the effect of the BT shunt seems to potentiate the risk of death or heart transplant in the subgroup of patients with the poorest myocardial reserve. A dilated right ventricle, an infectious disease complication in the post-Norwood period, and a surgeon who performed five or less Norwood procedure per year, influences the transplant-free survival for both the RVPA cohort and the BT shunt cohort. However, the cumulative effects of the risk factors was worst for the BT shunt group with predicted survival of $1 \%$ (70\% CI 0-13\%) at 6 months (Figure 30) with only 1 subjects surviving with this combination of risk factors. 


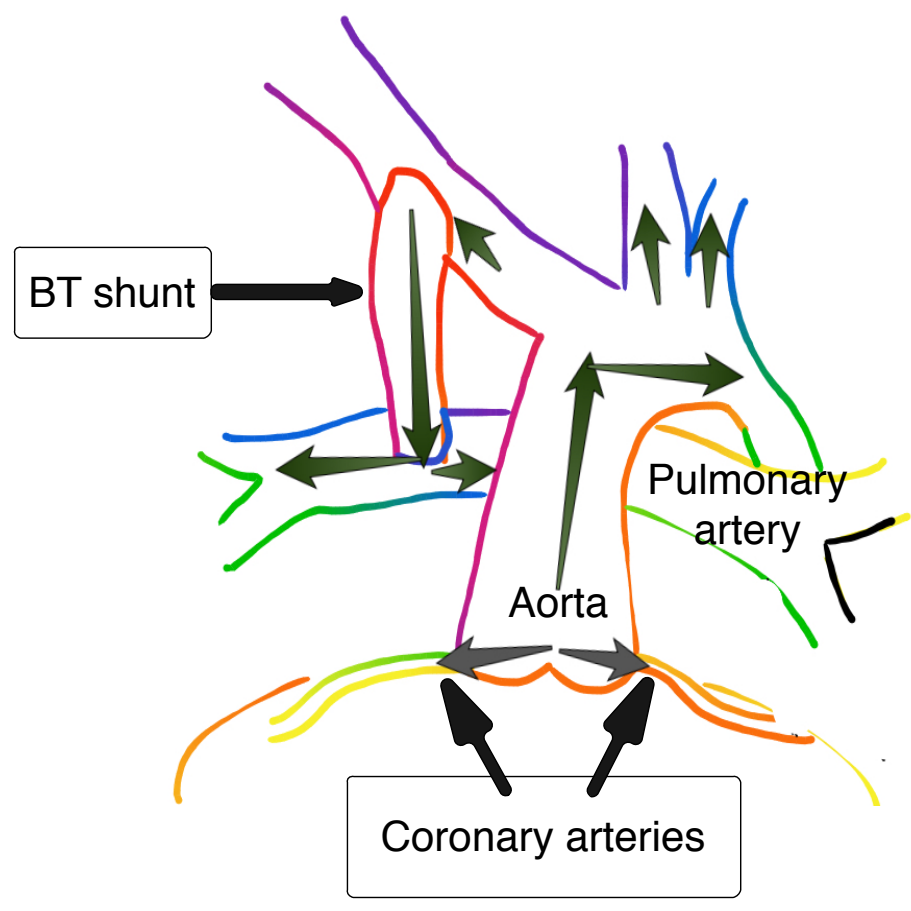

Figure 34. The BT shunt anatomy. Anatomy after BT shunt surgery showing that continuous flow through the BT shunt could compromise the blood flow through the coronary arteries since they are in parallel potentially leading to myocardial ischemia.

\subsubsection{The physiology associated with the BT shunt}

Why is shunt type such an important risk factors in this population? In reality, both shunt types can cause or potentiate the effect of ventricular dysfunction. The BT shunt allows for continuous flow into the pulmonary circulation, which leads to diastolic run off (Hsia et al. 2011). A diastolic run off means that flow to the pulmonary arteries continues in diastole instead of only occurring in systole (figure 34 and 35). This diastolic run off in turn reduces the diastolic pressure within the aorta (Bradley et al. 2004; Ghanayem et al. 2006; Donnelly et al. 1998; DeCampli et al. 2013). Coronary artery flow mainly occurs during diastole and is dependent on the aortic diastolic pressure as well as the pressure difference between aorta and the left ventricle (Driscol et al. 1964). The BT shunt can lead to lower coronary artery perfusion, which in turn can lead to myocardial ischemia (Donnelly et al. 1998) (Figure 34). This phenomenon could cause ventricular dysfunction secondary to myocardial cell death from ischemia. The 
consequences could also be worsened in the setting of ventricular dysfunction, which is often associated with higher ventricular diastolic pressure (Baird \& Adiseshiah 1976) and increased myocardial oxygen demand. The higher ventricular diastolic pressure results in a smaller difference in pressure between the aorta and the left ventricle during diastole. This may lead to worsening of coronary artery perfusion and, hence, myocardial perfusion. In addition, workload of the heart is often increased, as the ventricular geometry changes with ventricular dysfunction (Laskey et al. 1984). This change in workload and wall stress increases myocardial oxygen demand and may exacerbate the effect of the BT shunt on coronary artery perfusion. Finally, myocardial oxygen consumption is increased by medications usually employed for patients with symptomatic ventricular dysfunction such as inotropic medications. 

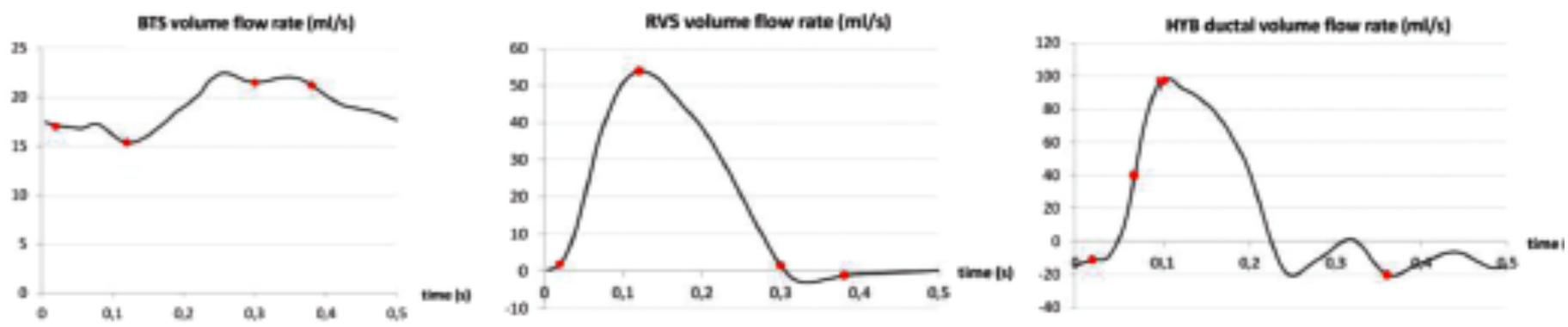

\section{BTS model}
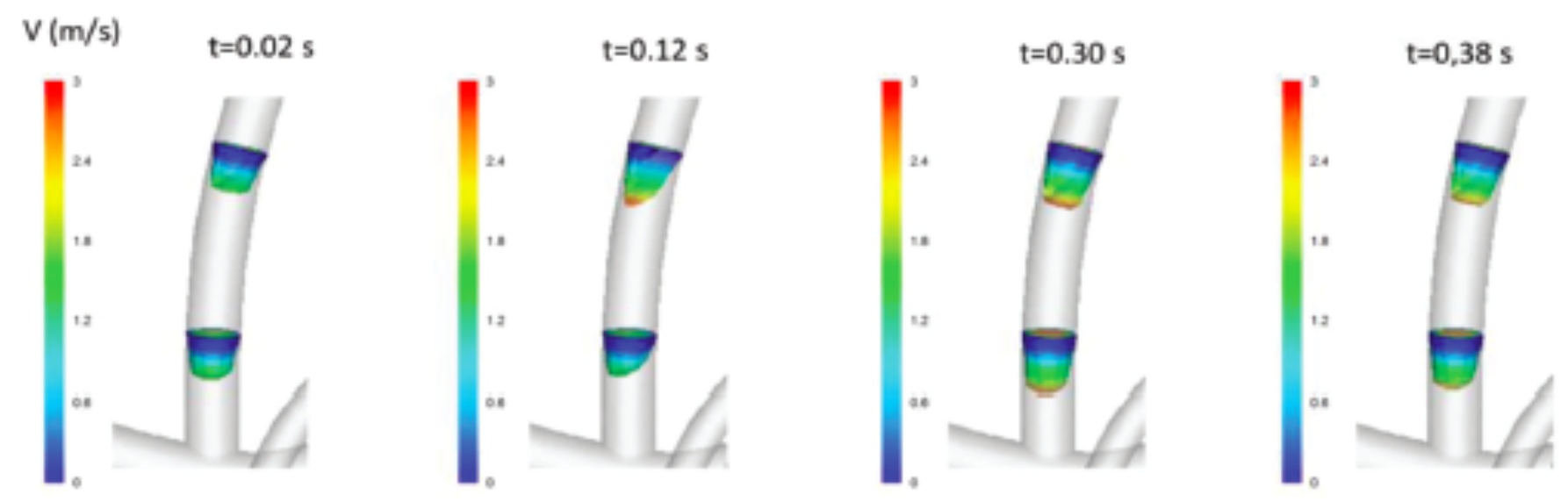

\section{RVS model}
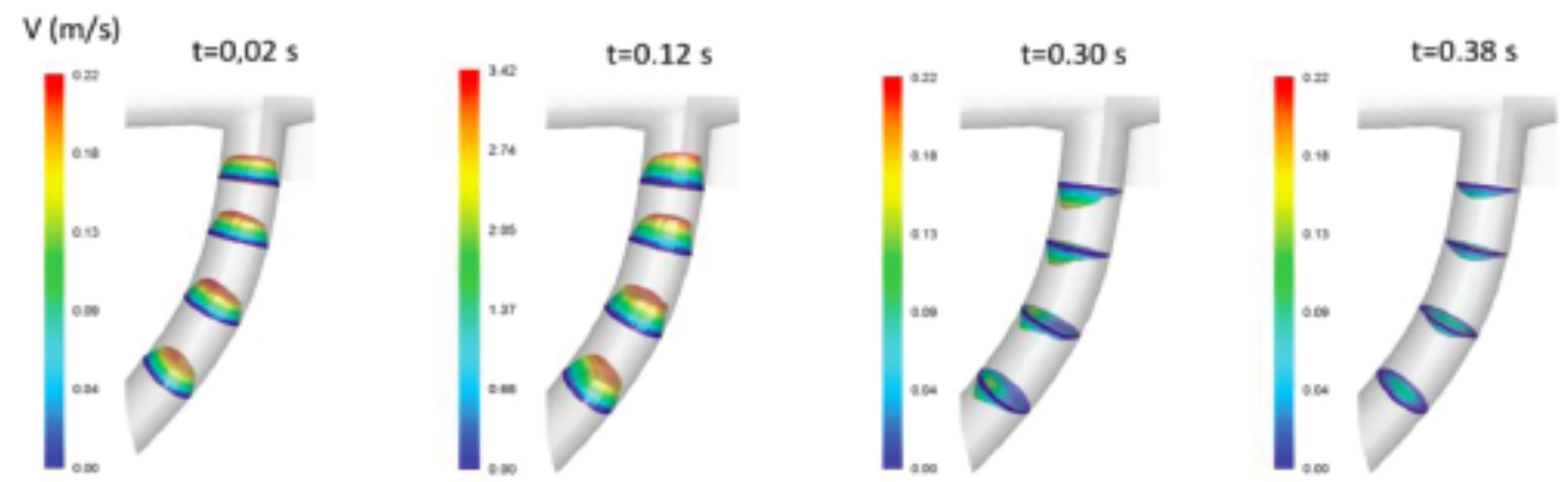

\section{HYB model}
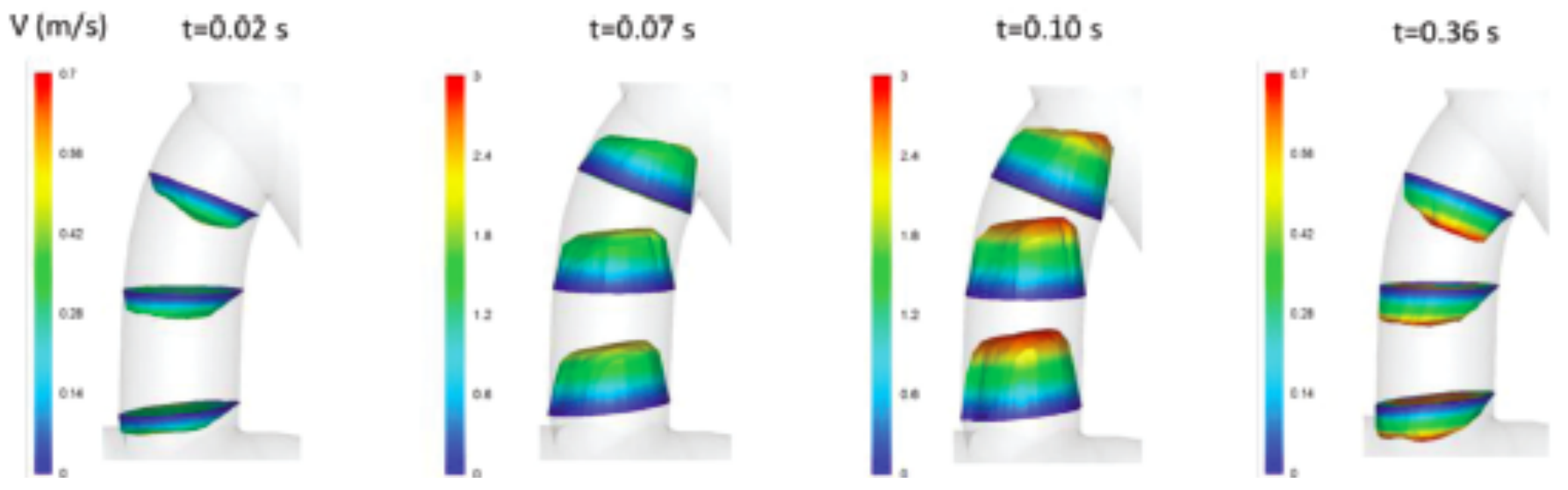
Figure 35. Solution of a mathematical model for BT shunt, RVPA conduit and Hybrid approach blood flow. The top series of panels demonstrate blood flow through BT shunt on the top left panel, the blood flow through RVPA conduit on the top middle panel and the blood flow through the PDA stent (top right panel) over one cardiac cycle. This is showing the continuous blood flow of the BT shunt (left top panel) compare to the blood blow in the RVPA conduit (middle top panel) that only occur in one part of the cardiac cycle, the systole. This is also showing the reverse blood flow that occurs in early diastole with the RVPA conduit (middle top panel) due to the associated conduit regurgitation. The three other series of panels demonstrate the solution of the three mathematical models used by the authors. Reproduced with permission from (Hsia et al. 2011), Copyright Wolters Kluwer Health Inc.

\subsubsection{The physiology associated with the RVPA conduit}

On the other hand, an incision into the right ventricular free wall, called a ventriculotomy, is necessary for implantation of the proximal end RVPA conduit (Figure 36). This leads to ventricular scarring (Wong et al. 2016). An autopsy study has demonstrated that the ventriculotomy scar is associated with increased fibrosis and thinning of the surrounding myocardium, and this effect does not seem to regress with time (Menon et al. 2013). Reverse remolding of the surrounding myocardium can further affect function, since myocardial cells are needed to generate ventricular wall thickening. In addition, the RVPA conduit is a valveless conduit directly connected to the ventricular cavity. The associated free regurgitation leads to increased volume loading of the ventricle and potentially progressive ventricular dilatation. Case series of serial cardiac MRIs comparing right ventricular volume and function measurements between the two shunt types (Wong et al. 2016) have shown that HLHS patients with a RVPA conduit had more dilation of the right ventricle compared to modified BT shunt patients, both after stage I and stage II surgery. There was also no difference between the ejection fraction measurements after the stage I procedure, but the RVPA patients had lower RVEF after stage II. Potentially, this points to the fact that the effect of the ventriculotomy scar on ventricular function is more likely a long-term issue. A scarred myocardium will not contract in the same way as the rest of the healthy myocardium (Menon et al. 2013). Depending on the severity and thickness of the myocardial injury, the myocardial contractions will be variably affected (Rácz et 
al. 2015). The myocardium can be hypokinetic, defined by less intense myocardial contraction with reduced radial motion but in the normal direction. The myocardium might not be moving at all with each cardiac contraction, called akinesia. Finally, if the myocardium injury affects the full thickness of the myocardium, it will usually move in the opposite direction. As the intraventricular pressure rises in systole, the thinned scar myocardium will dilate outward, called dyskinesia. These are all the forms of mechanical dyssynchrony, defined as an abnormal myocardial contractile motion, which has been shown to cause progressive ventricular dysfunction overtime (Dai et al. 2013; Lancellotti \& Moonen 2012). Therefore, the late worsening of function in the RVPA cohort might represents a progressive influence of the myocardial scar on ventricular function due to the associated mechanical dyssynchrony. Few studies have compared the ventricular function of the two shunt types. Using the Congenital Heart Surgeons Society (CHSS) cohort, Wilder et al. demonstrated that there was significantly more moderate right ventricle dysfunction within the modified BT shunt group in the first six months of life (Wilder, McCrindle et al. 2015b). However, after one year, there was no difference between the RVPA cohort and the BT shunt cohort. When the SVR trial investigators also looked at this question (Frommelt et al. 2012), they found that the right ventricular function was low for both groups (BT shunt and RVPA conduit) throughout their study. Similar to the CHHS cohort, they found that RVEF was significantly higher for the RVPA group (49 \% +/- 7 $\%$ vs. $44 \%+/-8 \%, \mathrm{p}<0.001)$ as well as RVFAC $(38 \%+/-7 \%$ vs. $35 \%+/-8 \%, \mathrm{p}<0.001)$ on the post-Norwood echocardiogram. They also showed no significant differences in RVEF and RVFAC on the pre-stage II echocardiogram and the last follow-up echocardiogram 14 months after randomization. Another secondary analysis of the SVR trial identified that having a BT shunt compare to RVPA conduit was an independent risk factor for ventricular dysfunction on the post-Norwood echocardiogram (Tabbutt et al. 2012). Another analysis of the SVR trial demonstrated an increase in late deaths and late heart transplants within the RVPA cohort while evaluating the three-year follow-up, and hypothesized that this was might be related to the longterm effect of the ventriculotomy (Newburger et al. 2014). However, this finding was not replicated for CHSS cohort, which showed a long-term survival advantage of the RVPA conduit over BT shunt and the hybrid procedure (Wilder et al. 2016) at the four-year follow-up. In our right ventricular dysfunction cohort, selected for abnormally low RVEF and RVFAC measurements on the post-Norwood echocardiogram, shunt type was not associated with a significant difference in RVEF and RVFAC measurement even though shunt type was a strong 
risk factor of death or heart transplant without achieving stage II procedure. We could not determine the long-term effects on ventricular function of the RVPA conduit since we only evaluated transplant-free survival to stage II. This might have artificially worsened the effect of BT shunt on survival. Analysis of the right ventricular dysfunction cohort transplant-free survival following the stage II procedure could assess the long-term effect of the RVPA conduit on survival and ventricular function. Nevertheless, having a BT shunt negatively influences the chance of transplant-free survival to the stage II procedure in patients with right ventricle dysfunction.
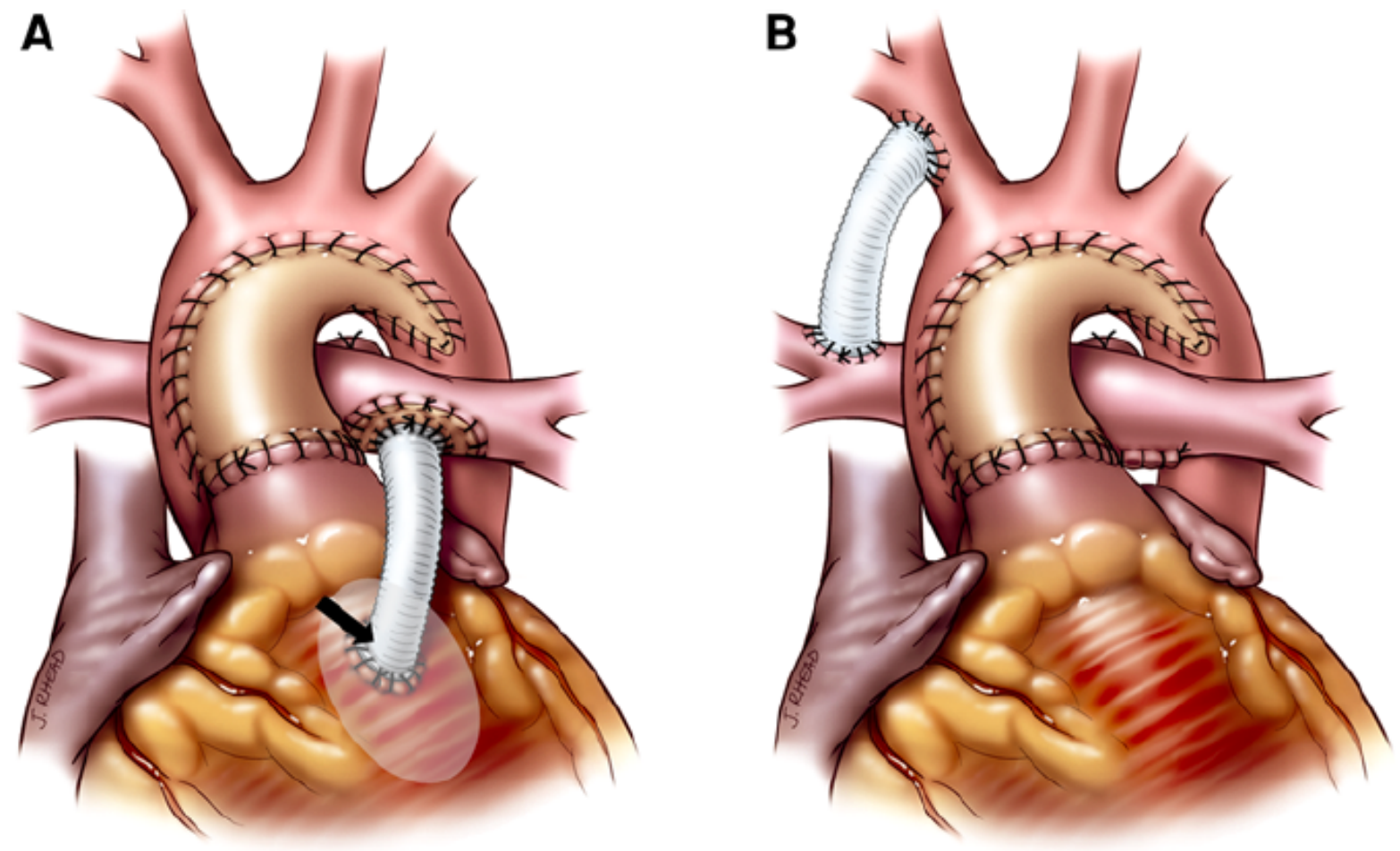

Figure 36. Diagram of the BT shunt and RVPA conduit surgeries. Panel A is a drawing of the surgical reconstruction associated with the RVPA conduit and the Norwood procedure. Panel B is a drawing of the surgical reconstruction associated with the BT shunt and the Norwood procedure. These drawings demonstrate that only the RVPA conduit requires a ventriculotomy, incision into the right ventricular free wall. Reprinted with permission from Macmillan Publishers Ltd: Pediatric Cardiology (Menon \& al.), copyright (2013). 


\subsection{Right ventricular dilatation}

\subsubsection{The Laplace law}

According to LaPlace's law (Gjesdal et al. 2011), the factors that determine myocardial wall stress greatly influence myocardial oxygen demands (Figure 37). The Laplace formula is as follows:

Wall stress $=$ ventricular pressure $*$ ventricular radius

$$
2 * \text { ventricular wall thickness }
$$

In other words, as the ventricle dilates, the wall stress increases and the workload of the heart increases. A more dilated ventricle that works harder for the same result would result in a decreased circulatory reserve. The physiology associated with ventricular dilation might explain why this is an independent risk factor for death or transplant in multiple other cardiac conditions (Alexander et al. 2012, Abe et al. 2013, Everitt et al. 2014, Therrien et al. 2005). 


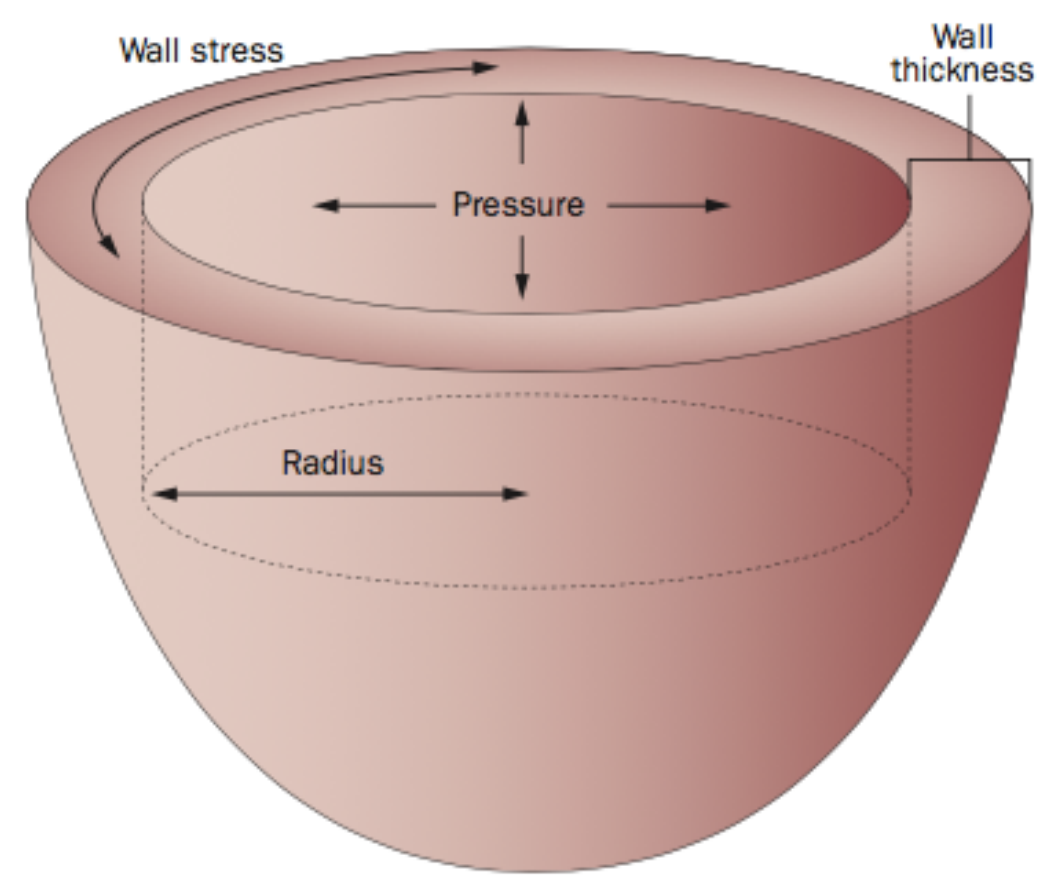

Figure 37. The Laplace law. The graphic shows the factors that influence wall stress and therefore myocardial workload. Reprinted with permission from Nature Publishing Group: Nature Reviews Cardiology (Gjesdal \& al.), copyright (2011).

\subsubsection{The relation between ventricular dysfunction and ventricular dilatation}

With progressive ventricular dysfunction, as the myocardium get sicker and more scarred, the ventricle will dilate. This phenomenon is called reverse remolding. Therefore, ventricular dilatation is usually observed when there is significant ventricular dysfunction. (Matsumura et al. 2013; Alexander et al. 2013) In other diseases, such as dilated cardiomyopathy, ventricular dilatation is one of the main predictors of outcome where ejection fraction is often not an important predictor (Everitt et al. 2014; Alexander et al. 2013). In other congenital diseases, such as Tetralogy of Fallot, right ventricular dilatation has also been shown to be one of the main outcome drivers (Therrien et al. 2005; Henkens et al. 2007). It is not surprising that ventricular dilation is an independent risk factor for HLHS patients with ventricular dysfunction. 


\subsubsection{The role of atrio-ventricular valve regurgitation}

Other factors such as the presence of AVVR influences the presence and severity of ventricular dilatation. AVVR is a general term that includes tricuspid regurgitation but also other valve regurgitation in the same position when the underlying anatomy of the patient leads to another valve in the tricuspid position such as in AVSD. Moderate to severe AVVR can cause or be caused by ventricular dilation. The volume loading associated with AVVR causes the ventricle to dilate. At the same time, as the ventricle dilates because of the ventricle dysfunction, it causes simultaneous dilatation of the valve annulus, which in turn affects the coaptation of the valve leaflets. This leads to the inability of the valve to efficiently close, exacerbating the insufficiency of the valve. AVVR has been shown to be associated with mortality and reinterventions in patients with HLHS (Honjo et al. 2011). For example, a matched case-control study looked at independent risk factors for death in a group of HLHS patients with moderate or greater AVVR (Honjo et al. 2011). They found that greater indexed atrioventricular valve annular size, increased cardiopulmonary bypass time and decreased post-repair ventricular function were all independent predictors of mortality. Interestingly, another analysis from the same group found that shunt types were not associated with the risk of developing AVVR (Chetan et al. 2013). In our analysis, either the presence or severity of tricuspid valve regurgitation was not associated with death or heart transplant without achieving the stage II procedure. In addition, the right ventricular end-diastolic size did not differ between patients who had tricuspid valve regurgitation on the post-Norwood echocardiogram versus patients who did not ((3.09 $\mathrm{cm}$ (IQR 2.69-3.19) vs. $3.01 \mathrm{~cm}(\mathrm{IQR} 2.81-3.20), \mathrm{p}=0.81$ ). Similarly, patient with at least moderate tricuspid valve regurgitation did not have bigger end-diastolic measurement of the right ventricle than patient without (2.89 $\mathrm{cm}$ (2.76-3.09) vs. $3.03 \mathrm{~cm}$ (IQR 2.83-3.25), p=0.11). However, all the patients in our cohort suffered from ventricular dysfunction and, therefore, had another reason to developed dilatation other than AVVR. This supports the premise that the negative effect on survival associated with right ventricle dilatation is secondary to its effects on myocardial performance and effects on myocardial reserve. 


\subsection{Surgeon volume}

Higher case volume has been associated with better outcomes in a wide range of surgical procedures (Shahian et al. 2015), and this has also been demonstrated in pediatric cardiac surgery (Pasquali et al. 2012). The volume-mortality relationship seems stronger for more complex surgery (Welke et al. 2009; Bazzani \& Marcin 2007) and the variation of outcomes between institutions has also been demonstrated (Jenkins et al. 1995). The effect of both institution volume and surgeon volume on Norwood procedure outcomes has been studied, but the influence still remains unclear. Using the Society of Thoracic Surgeons (STS) Congenital Heart Surgery database, Hornik et al. evaluated the effect of volume on outcomes post-Norwood procedure (Hornik et al. 2012). They found that both lower surgeon volume (OR for surgeons with $0-5$ cases vs. $>10 /$ year $=1.60(95 \%$ CI $1.12-2.27), \mathrm{p}=0.01)$ and lower center volume $((\mathrm{OR}$ for $0-10$ cases vs. $>20$ cases/year $=1.56(95 \%$ CI 1.05-2.31, $\mathrm{p}=0.03))$ were both associated with higher odds of mortality. Another group studied 801 Norwood procedures performed at 29 different hospitals across the United States and performed by 87 different surgeons (Checchia et al. 2005). They found that survival post-Norwood procedure was associated with center volume but not individual surgeon case volume. Similarly, Pasquali et al. found that lower center volume was associated with higher mortality post-Norwood procedure, and was independent of patients' individual risk factors (OR 1.54, 95\% CI 1.02-2.32, $\mathrm{p}=0.04$ ) (Pasquali et al. 2012). Karamlou et al. brought forward the idea that survival of neonates with complex congenital heart disease was more influenced by patient and management factors than center or surgeon volume (Karamlou et al. 2012). Using the Congenital Heart Surgeon Society multi-institutional studies, they were able to evaluate 2421 neonates for the association between institution performance and surgeon volume on outcomes for four groups of patients (transposition of the great arteries, pulmonary atresia with intact ventricular septum, Norwood procedure, and interrupted aortic arch). A significant variation in institution performance was demonstrated. They also found that surgeon experience seems to influence outcomes for transposition of the great arteries but not for the Norwood procedure. They concluded that the complexity of the post and preoperative care was much higher for the Norwood procedure than for the transposition of the great arteries patients potentially explaining the large influence of institutional experience rather than surgeon volume for the Norwood procedure patients. In the SVR trial, surgeon volume was identified as an independent risk factor in few of the study analyses. For example, surgeon volume was a 
predictor for post-Norwood morbidities, more precisely for renal failure, longer time to first extubation, and duration of ventilation (Tabutt et al. 2012). Lower center Norwood volume was associated with sepsis, longer time to first extubation, as well as increased duration of ventilation and length of hospital stay after the stage II procedure (Schwartz et al, 2014). Similarly, lower surgeon volume, but not center volume was also associated with lower three-year transplant-free survival (OR 1.73, 95\% CI 1.73 (1.05-2.85), $\mathrm{p}=0.001)$ (Newburger et al. 2014). This is consistent with our study finding that surgeon volume is a significant predictor of outcomes in our HLHS population with ventricular dysfunction. The SVR trial is unique because the patients were randomized between two different surgical procedures (Ohye et al. 2008), which obligated surgeons to performed a surgical procedure that they may not have been comfortable performing. Introducing a new surgical technique has its challenges (Bull et al. 2000). For example, when the arterial switch operation was introduced for transposition of the great arteries, the early mortality was much higher than where it currently stands (Hraska et al. 2003). The effect of the learning curve may be potentiated by low surgeon volume, and potentially explained why surgeon volume has been identified in analyses from the SVR trial as an independent risk factor (Newburger et al. 2014). In other hand, we could also conclude that surgical expertise and experience is required for this complex surgery.

\subsection{Infectious disease}

Infection in children post-congenital heart surgery is a frequent complication, with a reported incidence as high as 30 \% (Pollock et al. 1990; Pasquali et al. 2013; Ben-Ami et al. 2008; Algra et al. 2012). Some of the infections are superficial surgical wound infections. However, more rarely, patients will experience major infections, such as septicemia, mediastinitis, and endocarditis (Barker et al. 2010). Multiple groups have studied risk factors associated with developing an infection post-pediatric cardiac surgery. Algra et al. identified age less than six months, a post-operative ICU stay longer than 48 hours, and an open sternum for more than 48 hours as risk factors for infection (Algra et al. 2012). Costello et al. identified age younger than one year and longer cardiopulmonary bypass duration as independent risk factors for infection post-cardiac surgery (Costello et al. 2010). Ben-Ami et al. also found young age as a risk factor for wound infection, but also identified cyanotic heart disease and the presence of a indwelling 
catheter as an additional independent risk factors (Ben-Ami et al. 2008). HLHS patients are not spared from this complication. The Norwood procedure is performed shortly after birth, it is a complex surgery and it leads to an unstable circulation that requires often long and complex intensive care management. Therefore, most HLHS patients have risk factors for infection. In our ventricular dysfunction cohort, 50 (41\%) patients had an infectious disease complication, such as sepsis, respiratory infection, or urinary tract infection after the Norwood procedure. Interestingly, there was no difference between the ventricular dysfunction cohort and the normal function cohort in prevalence of infection complications (Table 1) post-Norwood procedure. Experiencing an infection post-cardiac surgery has been previously reported to be associated with morbidities and mortality. Barker et al. showed that major infections after cardiac surgery in children were associated with a higher chance of death (22\% (95\% CI $19-25 \%)$ vs. $3 \%$ (95\% CI $3-3 \%)$ ). They also shown that $70 \%(95 \%$ CI $67-73 \%)$ of the patients staying longer than 21 days in patients that experienced a major infection versus only $11 \%$ (95\% CI 10 - $11 \%$ ) of patients without major infections (Barker et al. 2010). Holzmann-Pazgal et al. similarly showed that surgical site infection was associated with longer ICU stays, longer hospital stays, and was associated with an increased risk of death after adjusting for illness severity (OR 4.9 (95\% CI 1.1-22.8), $\mathrm{p}=0.04$ ) (Holzmann-Pazgal et al. 2008).

\subsection{Unique analysis of a well-known dataset}

The SVR trial has led to multiple publications, and the risk factors for death and transplant have been studied over different periods of time. In addition, the effect of shunt types on ventricular function in the SVR cohort has been evaluated (Frommelt et al. 2012). However, our study is unique in that it evaluated the subgroup of patients with ventricular dysfunction, a subgroup of patients previously not differentiated. It is clear that the presence of ventricular dysfunction affects outcome. However, the prevalence and influence of ventricular dysfunction seems to vary depending on the observation period assessed. For example, ventricular dysfunction was not a risk factor for death or heart transplant at discharge post-Norwood, at thirty-days post-Norwood (Tabbutt et al. 2012), during the interstage period (Ghanayem et al. 2012), during the stage II hospitalization (Schwartz et al. 2014), or at the 12-month follow-up (Ohye et al. 2010). However, it was a risk factor for death or heart transplant at 14 months post-randomization by 
competing risk analysis (Tweddell et al. 2012) and at three-year post-randomization (Newburger et al. 2014). Ventricular dysfunction is one of the more common reasons for transplant listing in infants with HLHS (Carlo et al. 2016; Kulkarni et al. 2016). As previously noted, ventricular dysfunction is not necessarily a permanent diagnosis, since some patients will improve or recover over time (Jean-St-Michel et al. 2016). Therefore, individually assessing risk factors for patients with ventricular dysfunction may provide insight into its impact on outcomes, but mainly helps clinician make decisions for this higher risk population, such as early transplant listing.

For the purpose of our study, we assessed the ability of this population to reach stage II palliation in order to help with decision making relating to heart transplant listing. We included the period of time from the Norwood procedure to the stage II procedure, which combined hospital morality, thirty-day mortality, and the interstage mortality into one time period. By employing multiphase parametric hazard modeling, we wanted to identify if the hazard of death or heart transplant varied over time, as well as identifying different risk factors for death or transplant associated with each risk phases (Sergeant, Blackstone \& Meyns 1997; Blackstone et al. 1986). Despite combining different risk periods into the time to event analysis, we identified only a single hazard phase, a constant phase. This was different to the analysis performed by Tweddell \& al. (Tweddell et al. 2012), for which the hazard of death or transplant to four years post-randomization was characterized by an early phase and a constant phase. However, their analysis included the entire SVR cohort and did not evaluate patients with ventricular dysfunction separately. Similarly to Tweddell \& al. (Tweddell et al. 2012), we employed parametric hazard modeling, which has rarely been employed with this dataset. An advantage of parametric hazard modeling is that the effects of the identified risk factors can be portrayed graphically while adjusting for the other risk factors. These prediction plots and nomograms provide visual information regarding the weight of the risk factors on the outcomes. This can potentially help clinician make decisions, since the impact of the risk factors are depicted graphically, which makes it easier to comprehend and potentially increases the knowledge regarding a particular patient predicted outcome. 


\subsection{Future directions}

This study identified an important modifiable risk factor for death or heart transplant, the effect of shunt type on survival in HLHS patients with ventricular dysfunction post-Norwood procedure. We demonstrated that a BT shunt negatively influences survival for this cohort of patients. Multiple physiological processes might explain the influence of BT shunt on survival in patients with ventricular dysfunction and none of them were evaluated with this analysis. Physiologic assessments of the effect of the BT shunt on coronary artery perfusion and, hence, myocardial perfusion in the setting of ventricular dysfunction would be of importance, as well as the role played by increased wall stress. In addition, replicating this analysis within another dataset, such as the CHSS's critical left ventricular outflow tract obstruction inception cohort, might confirm the negative impact of BT shunts on patients with ventricular dysfunction postNorwood procedure. This would confirm that patients with a BT shunt and ventricular dysfunction who have any other identified risk factors should be listed for heart transplant as early as possible in order to improve their chances of a successful heart transplantation.

\subsection{Study limitations}

\subsubsection{Selection bias}

Our study is a secondary data analysis of a dataset from a rigorous randomized clinical trial. The population was highly selected and the allocation of shunt was by chance only (Ohye et al. 2008a). However, we had limited control over the selection methods available to us within the SVR cohort. One of the main limitations of this study is the possibility of selection bias. We hoped that by requiring both measurements, RVEF and RVAFC, for entry into the study cohort, we would only select patients who truly have right ventricular dysfunction reducing the bias from inappropriate selection of controls, a form of selection bias. The timing of the echocardiograms was protocolized in the trial and limited the data availability. We had access to echocardiograms done before the Norwood procedure, post-Norwood procedure, and at the hospitalization for the stage II procedure (Frommelt et al. 2012). In addition, as discussed 
previously, the timing of the post-Norwood echocardiogram varied within the SVR cohort. This can lead to selection bias, as some patients may have developed ventricular dysfunction between the baseline and post-Norwood echocardiogram but showed improvement of ventricular function prior to the post-Norwood echocardiogram. Those patients would not be included in our study cohort since within the information available in the dataset no other assessment time point could be included. This form of bias could have artificially worsen the outcome of our ventricular dysfunction by excluding the patient that had a quick spontaneous improvement in their ventricular function. However, those patients might be inherently different than our ventricular dysfunction cohort and would have included to much variation within our study cohort.

\subsubsection{Information bias}

One of the advantages of the SVR protocol was that the echocardiogram protocols were standardized at each site. Furthermore, the echocardiograms were read at a core lab, which leads to improved quality of the ventricular function information available and reduced the potential information bias associated with measurement of right ventricular function. However, despite the fact that the echocardiograms were protocolized within the SVR trial, right ventricular function remains a challenging measurement. It requires good windows and specific views (Frommelt et al, 2012). This might explain why $22 \%$ (118 infants) of the entire SVR cohort had missing RVEF or RVFAC measurements on the post-Norwood echocardiogram. Those infants were excluded from both the normal function and ventricular dysfunction cohort in order to avoid misclassification. The fact that we excluded this big group of patients represents a form of information bias caused by the instrument of measurement. However, the main reasons for unavailability of the right ventricular function measurements in those patients were a limited study (the require views were not included in the study), a poor quality study and poor window leading to the inability of the core lab to do the measurements. Therefore, it is unlikely that the cohort of patients excluded was inherently different than the patients included in the analysis. 


\subsubsection{Confounding}

We are aware that they may have been some unknown confounders that we were unable to adjust for, since statistical methodology cannot account for unknown confounders. However, this is less likely to have influence the association between risk of death or heart transplant and shunt types since, as part of the SVR trial, patients were randomized to the two different shunt types.

\subsubsection{Left censoring}

As discussed in the method section, there was a potential for left censoring to influence our results. Our study cohort was selected based on measured right ventricular function on the postNorwood echocardiogram, but the timing of these echocardiograms was variable within our study cohort (Figure 16). In addition, the risk of death and heart transplant as well as the risk of developing ventricular dysfunction starts immediately post-Norwood procedure. Therefore, the period of observation may be artificially shortened because a patient might develop ventricular dysfunction a long time before the post-Norwood echocardiogram is performed (Figure 8). We adjusted for left censoring by including the period of risk between the Norwood procedure and the post-Norwood echocardiogram and making assumption on the distribution of risk (Figure 9). This analytic method was strengthen by the fact that none of the patients in the study cohort had right ventricular dysfunction on their baseline echocardiogram based on our inclusion criteria.

\subsubsection{Power}

Since RVEF was a missing variable in $22 \%$ subjects enrolled in the SVR trial, we were left with only 123 infants in the ventricular dysfunction cohort. Therefore, this analysis was potentially underpowered to identify other risk factors associated with death and heart transplant, since there were only 23 events. 


\subsection{Clinical implication}

The study may potentially influence the clinical decision regarding which patient to refer for heart transplants as well as influencing the timing of heart transplant's referrals. This study suggests that early heart transplant referral may not be considered if the patients who only have a BT shunt as a risk factor. However, early heart transplant listing may be considered in patients with right ventricular dysfunction, a BT shunt and other risk factors for death even if their ventricular dysfunction is only mild. In addition, one might consider more strongly to recommend an RVPA conduit over a BT shunt at time of the Norwood procedure in patients who are thought at risk of developing ventricular dysfunction.

\subsection{Conclusion}

In conclusion, in HLHS patient with ventricular dysfunction post-Norwood procedure have worse transplant free survival to stage II procedure than HLHS infant with normal ventricular function. Having a modified BT shunt was strongly associated with a decreased transplant free survival to the stage II procedure. Having a dilated right ventricle, experiencing an infectious disease complication post-Norwood, or having the Norwood procedure performed by a surgeon who performed 5 or less Norwood per year were additional independent risk factors for death or transplant. This analysis demonstrated that early transplant listing should be contemplated in patients with a BT shunt who develop right ventricular dysfunction, especially if other risk factors for death are present. 


\section{Tables}

\section{Table 1. Predictor variables included in the analysis}

\begin{tabular}{|c|c|c|c|}
\hline Predictor variables & Units & $\mathbf{N}$ & $\begin{array}{c}\text { Missing } \\
(\%)\end{array}$ \\
\hline \multicolumn{4}{|l|}{ Demographic: } \\
\hline Male & $\%$ & 365 & 0 \\
\hline Birth weight & g & 365 & 0 \\
\hline Presence of low birth weight & yes/no & 365 & 0 \\
\hline Birth weight group & & 365 & 0 \\
\hline$<30$ th percentile & yes/no & & \\
\hline 30-70th percentile & yes/no & & \\
\hline >70th percentile & yes/no & & \\
\hline Gestational age & weeks & 365 & 0 \\
\hline Premature birth & yes/no & 365 & 0 \\
\hline Prenatal diagnosis & yes/no & 365 & 0 \\
\hline Fetal intervention & yes/no & 280 & 24 \\
\hline HLHS classic anatomy & yes/no & 365 & 0 \\
\hline Cardiac diagnosis & & 365 & 0 \\
\hline HLHS with aortic atresia/mitral atresia & yes/no & & \\
\hline HLHS with aortic atresia/mitral stenosis & yes/no & & \\
\hline HLHS with aortic stenosis/mitral stenosis & yes/no & & \\
\hline HLHS with aortic stenosis/mitral atresia & yes/no & & \\
\hline Critical aortic stenosis & yes/no & & \\
\hline Single RV with systemic outflow obstruction & yes/no & & \\
\hline Right dominant AVSD & yes/no & & \\
\hline Straddling mitral valve with LV hypoplasia & yes/no & & \\
\hline \multicolumn{4}{|l|}{ Other functional single RV } \\
\hline Presence of aortic atresia & & 365 & 0 \\
\hline Presence of a right subclavian artery & yes/no & 359 & 1 \\
\hline Presence of pulmonary venous return obstruction & yes/no & 365 & 0 \\
\hline Presence of total anomalous pulmonary venous connection & yes/no & 365 & 0 \\
\hline Presence of partial anomalous pulmonary venous connection & yes/no & 365 & 0 \\
\hline \multicolumn{4}{|l|}{ Prior to the Norwood procedure: } \\
\hline Highest lactate & $\mathrm{mmol} / \mathrm{L}$ & 284 & 22 \\
\hline Intubated & yes/no & 365 & 0 \\
\hline Inhaled nitric gases & yes/no & 365 & 0 \\
\hline
\end{tabular}




\begin{tabular}{|c|c|c|c|}
\hline ECMO pre-Norwood & yes/no & 359 & 1 \\
\hline Any complications & yes/no & 365 & 0 \\
\hline Cardiac complications (RV dysfunction, arrhythmia, etc.) & yes/no & 365 & 0 \\
\hline Respiratory complications (pleural effusion, pneumothorax, etc.) & yes/no & $65^{3}$ & 0 \\
\hline Neurological complications (stroke, bleed, seizure, etc.) & yes/no & 365 & 0 \\
\hline GI complications (necrotizing enterocolitis, liver failure) & yes/no & 365 & 0 \\
\hline ID complication (sepsis, respiratory infection, UTI, etc.) & yes/no & 365 & 0 \\
\hline Renal complication (acute or chronic renal failure) & yes/no & 365 & 0 \\
\hline Other complication (hematological, etc.) & yes/no & 365 & 0 \\
\hline Randomized to modified BT shunt & yes/no & 365 & 0 \\
\hline \multicolumn{4}{|l|}{ Norwood hospitalisation: } \\
\hline Surgeon volume & yes/no & 365 & 0 \\
\hline Year of Norwood & & 365 & 0 \\
\hline 2005 & yes/no & & \\
\hline 2006 & yes/no & & \\
\hline 2007 & yes/no & & \\
\hline 2008 & yes/no & & \\
\hline Age at Norwood & days & 365 & 0 \\
\hline Length of ICU stay & days & 365 & 0 \\
\hline Length of stay & days & 365 & 0 \\
\hline Modified BT shunt at end of procedure & yes/no & 365 & 0 \\
\hline Diameter of modified BT shunt & $\mathrm{mm}$ & 171 & 53 \\
\hline Length of the modified BT shunt & $\mathrm{mm}$ & 160 & 56 \\
\hline Diameter of the RVPA conduit & $\mathrm{mm}$ & 195 & 46 \\
\hline Length of RVPA conduit & $\mathrm{mm}$ & 156 & 57 \\
\hline Total cross clamp time & $\min$ & 365 & 0 \\
\hline Bypass time & $\min$ & 359 & 1 \\
\hline Deep hypothermic circulatory arrest time was used & yes/no & 365 & 0 \\
\hline Deep hypothermic circulatory arrest time & $\min$ & 365 & 0 \\
\hline Regional cerebral perfusion used & yes/no & 363 & 1 \\
\hline Regional cerebral perfusion time & $\min$ & 151 & 58 \\
\hline Regional cerebral perfusion flow & $\mathrm{cc} / \mathrm{kg} / \mathrm{min}$ & 149 & 59 \\
\hline Lowest temperature recorded during Norwood & celcius & 365 & 0 \\
\hline Lowest hematocrit recorded during Norwood & $\mathrm{mmol} / \mathrm{L}$ & 363 & 1 \\
\hline Ultrafiltration used during bypass & yes/no & 307 & 16 \\
\hline Ultrafiltration used after bypass & yes/no & 272 & 25 \\
\hline Steroids given prior to Norwood & yes/no & 359 & 1 \\
\hline Aprotinin was given & yes/no & 359 & 1 \\
\hline Alpha blockade was given & yes/no & 359 & 1 \\
\hline Type of alpha blockade & & 164 & 55 \\
\hline Phenozybenzamine & yes/no & & \\
\hline
\end{tabular}




\begin{tabular}{|c|c|c|c|}
\hline Phentolamine & yes/no & & \\
\hline Coarctectomy & yes/no & 363 & 1 \\
\hline Patch repair of aortic arch & yes/no & 363 & 1 \\
\hline Other procedure done at time of Norwood & yes/no & 365 & 0 \\
\hline ECMO post-Norwood & yes/no & 365 & 0 \\
\hline CPR post-Norwood & yes/no & 365 & 0 \\
\hline Age at extubation & days & 364 & 1 \\
\hline Duration of ventilation & days & 364 & 1 \\
\hline Open sternum after leaving the operating room & yes/no & 365 & 0 \\
\hline Any complications post-Norwood & yes/no & 365 & 0 \\
\hline Number of post-Norwood complications & $\mathrm{n}$ & 365 & 0 \\
\hline Post-Norwood complication & yes/no & 365 & 0 \\
\hline Cardiac complications (RV dysfunction, arrhythmia, etc.) & yes/no & 365 & 0 \\
\hline Respiratory complications (pleural effusion, pneumothorax, etc.) & yes/no & 365 & 0 \\
\hline Neurological complications (stroke, bleed, seizure, etc.) & yes/no & 365 & 0 \\
\hline GI complications (necrotizing enterocolitis, liver failure) & yes/no & 365 & 0 \\
\hline ID complication (sepsis, repertory infection, UTI, etc.) & yes/no & 365 & 0 \\
\hline Renal complication (acute or chronic renal failure) & yes/no & 365 & 0 \\
\hline Other complication (hematological, etc.) & yes/no & 365 & 0 \\
\hline Discharge home pre-stage II without receiving a heart transplant & yes/no & 365 & 0 \\
\hline Oxygen saturation at discharge & $\%$ & 331 & 9 \\
\hline Oxygen at discharge & yes/no & 333 & 9 \\
\hline Number of medications at discharge & $\mathrm{n}$ & 350 & 4 \\
\hline Discharged on an ACE-inhibitor & yes/no & 350 & 4 \\
\hline Discharged on a Beta-blocker & yes/no & 350 & 4 \\
\hline Discharged on Inotrope & yes/no & 350 & 4 \\
\hline Discharged on a diuretic & yes/no & 350 & 4 \\
\hline Discharged with an anti-arrhythmic & yes/no & 350 & 4 \\
\hline Discharged on anti-hypertensive medication & yes/no & 350 & 4 \\
\hline Able to proceed to stage II & yes/no & 350 & 4 \\
\hline Died without achieving stage II & yes/no & 365 & 0 \\
\hline Received a heart transplant without achieving stage II & yes/no & 365 & 0 \\
\hline \multicolumn{4}{|l|}{ Baseline echocardiogram } \\
\hline \multicolumn{4}{|l|}{ Anatomical information: } \\
\hline Patent mitral valve & yes/no & 363 & 1 \\
\hline Patent aortic valve & yes/no & 361 & 1 \\
\hline Left ventricle present & yes/no & 363 & 1 \\
\hline Native ascending aorta dimension & $\mathrm{cm}$ & 356 & 2 \\
\hline Left pulmonary artery dimension & $\mathrm{cm}$ & 327 & 10 \\
\hline ASD Doppler gradient & $\mathrm{mmHg}$ & 220 & 40 \\
\hline \multicolumn{4}{|l|}{ RV and TV measurements: } \\
\hline RV end-diastolic long axis dimension & $\mathrm{mm}^{2}$ & 339 & 7 \\
\hline
\end{tabular}




\begin{tabular}{|c|c|c|c|}
\hline RV end-diastolic short axis dimension & $\mathrm{mm}^{2}$ & 339 & 7 \\
\hline RV ejection fraction & $\%$ & 292 & 20 \\
\hline RV fractional area of change & $\%$ & 338 & 7 \\
\hline RV eccentricity & & 339 & 7 \\
\hline RV isovolumic time & msec & 151 & 59 \\
\hline RV indexed end-diastolic volume & $\mathrm{mL}^{3} / \mathrm{BSA}^{*} 1.3$ & 254 & 30 \\
\hline RV indexed end-diastolic area & $\mathrm{mL}^{3} / \mathrm{BSA}^{*} 1.3$ & 338 & 7 \\
\hline Tissue Doppler: RV peak systolic velocity & $\mathrm{mmHg}$ & 151 & 59 \\
\hline Anteroposterior TV annulus & $\mathrm{cm}$ & 336 & 8 \\
\hline Anteroposterior TV annulus z score & z score & 336 & 8 \\
\hline Transverse TV annulus & $\mathrm{cm}$ & 357 & 2 \\
\hline Transverse TV annulus z score & z score & 357 & 1 \\
\hline TV annulus area & $\mathrm{mm}$ & 331 & 9 \\
\hline TV indexed annular area & $\mathrm{mm}^{2} / \mathrm{BSA}$ & 331 & 9 \\
\hline TV annular area z score & z score & 331 & 9 \\
\hline TV regurgitation & yes/no & 357 & 8 \\
\hline TV regurgitation severity & & 292 & 20 \\
\hline Mild & yes/no & & \\
\hline Moderate & yes/no & & \\
\hline Severe & yes/no & & \\
\hline \multicolumn{4}{|l|}{ Outflow tracts: } \\
\hline Aortic valve regurgitation & yes/no & 271 & 26 \\
\hline Aortic valve regurgitation severity & & 19 & 95 \\
\hline Mild & yes/no & & \\
\hline Moderate & yes/no & & \\
\hline Severe & yes/no & & \\
\hline Neoaortic valve regurgitation & yes/no & 360 & 1 \\
\hline Neoaortic valve regurgitation severity & & 85 & 77 \\
\hline Mild & yes/no & & \\
\hline Moderate & yes/no & & \\
\hline Severe & yes/no & & \\
\hline \multicolumn{4}{|l|}{ LV measurements: } \\
\hline LV end-diastolic long axis dimension & $\mathrm{Cm}$ & 240 & 34 \\
\hline LV end-diastolic volume & $\mathrm{mL}$ & 240 & 34 \\
\hline LV end-diastolic volume & $\mathrm{mL}$ & 240 & 34 \\
\hline LV ventricular mass & G & 207 & 43 \\
\hline LV ejection fraction & $\%$ & 220 & 40 \\
\hline Mitral valve regurgitation & yes/no & 184 & 50 \\
\hline Mitral valve regurgitation severity & & 85 & 76 \\
\hline Mild & yes/no & & \\
\hline Moderate & yes/no & & \\
\hline Severe & yes/no & & \\
\hline
\end{tabular}




\begin{tabular}{|c|c|c|c|}
\hline \multicolumn{4}{|l|}{ Post-Norwood echocardiogram } \\
\hline \multicolumn{4}{|l|}{ Anatomical information: } \\
\hline Narrowest distal arch diameter & $\mathrm{cm}$ & 343 & 6 \\
\hline ASD Doppler gradient & $\mathrm{mmHg}$ & 343 & 6 \\
\hline \multicolumn{4}{|l|}{ RV and TV measurements: } \\
\hline Anteroposterior TV annulus & $\mathrm{cm}$ & 359 & 2 \\
\hline Anteroposterior TV annulus z score & z score & 359 & 2 \\
\hline Transverse TV annulus & $\mathrm{cm}$ & 364 & 1 \\
\hline Transverse TV annulus z score & z score & 364 & 1 \\
\hline TV indexed annular area & $\mathrm{mm}^{2} / \mathrm{BSA}$ & 359 & 1 \\
\hline TV annular area $z$ score & z score & 359 & 1 \\
\hline TV regurgitation & yes/no & 365 & 0 \\
\hline TV regurgitation severity & & 325 & 11 \\
\hline Mild & yes/no & & \\
\hline Moderate & yes/no & & \\
\hline Severe & yes/no & & \\
\hline Worsening TV regurgitation & yes/no & 357 & 8 \\
\hline RV end-diastolic long axis dimension & $\mathrm{mm}^{2}$ & 338 & 7 \\
\hline RV end-diastolic short axis dimension & $\mathrm{mm}^{2}$ & 338 & 7 \\
\hline RV ejection fraction & $\%$ & 365 & 0 \\
\hline Worsening RV ejection fraction & yes/no & 292 & 20 \\
\hline $\mathrm{RV}$ fractional area of change & $\%$ & 365 & 0 \\
\hline Worsening RV fractional area of change & yes/no & 338 & 7 \\
\hline RV eccentricity & & 365 & 0 \\
\hline RV isovolumic time & msec & 51 & 85 \\
\hline RV indexed end-diastolic volume & $\mathrm{mL}^{3} / \mathrm{BSA}^{*} 1.3$ & 365 & 0 \\
\hline RV indexed end-diastolic area & $\mathrm{mL}^{3} / \mathrm{BSA}^{*} 1.3$ & 365 & 0 \\
\hline Tissue Doppler: RV peak systolic velocity & $\mathrm{mmHg}$ & 254 & 30 \\
\hline \multicolumn{4}{|l|}{ Outflow tracts: } \\
\hline Aortic valve regurgitation & yes/no & 120 & 67 \\
\hline Aortic valve regurgitation severity & & 17 & 95 \\
\hline Mild & yes/no & & \\
\hline Moderate & yes/no & & \\
\hline Severe & yes/no & & \\
\hline Neoaortic valve regurgitation & yes/no & 365 & 0 \\
\hline Neoaortic valve regurgitation severity & & 175 & 52 \\
\hline Mild & yes/no & & \\
\hline Moderate & yes/no & & \\
\hline Severe & yes/no & & \\
\hline \multicolumn{4}{|l|}{ LV measurements: } \\
\hline Mitral valve regurgitation & yes/no & 364 & 1 \\
\hline Mitral valve regurgitation severity & & 162 & 56 \\
\hline
\end{tabular}




\begin{tabular}{|l|c|c|c|} 
Mild & yes/no & & \\
\hline Moderate & yes/no & & \\
\hline Severe & yes/no & & \\
\hline LV ejection fraction & $\%$ & 220 & 40 \\
\hline
\end{tabular}

Abbreviations: HLHS, hypoplastic left heart syndrome; RV, right ventricle; AVSD, atrioventricular septal defect; LV, left ventricle; ECMO, extracorporeal membrane oxygenation; GI, gastrointestinal; ID, infectious disease; UTI, urinary tract infection; BT, Blalock-Taussig; ICU, intensive care unit; RVPA, right ventricle to pulmonary artery; CPR, cardiopulmonary resuscitation; ACE, angiotensin-converting enzyme; ASD, atrial septal defects; TV, tricuspid valve; BSA, body surface area; $\mathrm{g}$, gram; $\mathrm{mm}$, millimeter; $\mathrm{mmol} / \mathrm{L}$, millimole per liter; min, minute; $\mathrm{cc} / \mathrm{kg} / \mathrm{min}$, milliliter per kilogram per minute; $\mathrm{n}$, number; $\mathrm{cm}$, centimeter; $\mathrm{mmHg}$, millimeter of mercury; $\mathrm{mL}$, milliliter. 


\section{Table 2. Demographic and Norwood procedure hospitalization} information for the ventricular dysfunction and the normal function cohorts.

\begin{tabular}{|c|c|c|c|c|}
\hline & \begin{tabular}{|l} 
All patients \\
with RVEF or \\
RVFAC \\
measurements \\
$(n=365)$
\end{tabular} & $\begin{array}{l}\text { Ventricular } \\
\text { dysfunction } \\
\text { cohort ( } n=123)\end{array}$ & $\begin{array}{l}\text { Normal } \\
\text { function cohort } \\
\text { ( } n=242)\end{array}$ & P-value \\
\hline \multicolumn{5}{|l|}{ Demographic: } \\
\hline Male: n (\%) & $224(61)$ & $67(55)$ & $157(65)$ & 0.05 \\
\hline Birth weight (g): mean (SD) & $3133.7(523.1)$ & $3163.1(525.1)$ & $3118.8(522.6)$ & 0.44 \\
\hline Presence of low birth weight: $\mathrm{n}(\%)$ & $38(10)$ & $14(11)$ & $24(10)$ & 0.66 \\
\hline Birth weight group: $\mathrm{n}(\%)$ & & & & 0.34 \\
\hline$<30$ th percentile & $185(51)$ & $56(46)$ & $129(53)$ & \\
\hline 30-70th percentile & $126(35)$ & $48(39)$ & $78(32)$ & \\
\hline$>70$ th percentile & $54(15)$ & $19(15)$ & $35(14)$ & \\
\hline Gestational age (weeks): median (IQR) & 38 (38-39) & $38(38-39)$ & $38(38-39)$ & 0.77 \\
\hline Premature birth: $\mathrm{n}(\%)$ & $35(10)$ & $10(8)$ & $25(10)$ & 0.50 \\
\hline Prenatal diagnosis: $\mathrm{n}(\%)$ & $279(76)$ & $92(75)$ & $187(77)$ & 0.60 \\
\hline Fetal intervention: $\mathrm{n}(\%)$ & $9(2)$ & $1(1)$ & $8(3)$ & 0.36 \\
\hline HLHS classic anatomy: $n(\%)$ & $319(87)$ & $111(90)$ & $208(86)$ & 0.24 \\
\hline Cardiac diagnosis: $\mathrm{n}(\%)$ & & & & 0.78 \\
\hline HLHS with aortic atresia/mitral atresia & $142(39)$ & $48(39)$ & $94(39)$ & \\
\hline HLHS with aortic atresia/mitral stenosis & $86(24)$ & $33(27)$ & $53(22)$ & \\
\hline HLHS with aortic stenosis/mitral stenosis & $84(23)$ & $27(22)$ & $57(24)$ & \\
\hline HLHS with aortic stenosis/mitral atresia & $7(2)$ & $3(2)$ & $4(2)$ & \\
\hline Critical aortic stenosis & $2(1)$ & $0(0)$ & $2(1)$ & \\
\hline $\begin{array}{l}\text { Single RV with systemic outflow } \\
\text { obstruction }\end{array}$ & $13(4)$ & $4(3)$ & $9(4)$ & \\
\hline Right dominant AVSD & $21(6)$ & $4(3)$ & $17(7)$ & \\
\hline $\begin{array}{c}\text { Straddling mitral valve with LV } \\
\text { hypoplasia }\end{array}$ & $1(0.3)$ & $0(0)$ & $1(0.5)$ & \\
\hline Other functional single RV & $9(3)$ & $4(3)$ & $5(2)$ & \\
\hline Presence of aortic atresia: $\mathrm{n}(\%)$ & $225(62)$ & $74(60)$ & $151(62)$ & 0.68 \\
\hline Ascending aorta size $(\mathrm{mm})$ : median (IQR) & $3.0(2.0-4.0)$ & $2.7(2.0-3.5)$ & $3.0(2.0-4.0)$ & 0.26 \\
\hline Presence of a right subclavian artery: $n(\%)$ & $21(7)$ & $4(3)$ & $17(7)$ & 0.11 \\
\hline $\begin{array}{l}\text { Presence of pulmonary venous return } \\
\text { obstruction: } \mathrm{n}(\%)\end{array}$ & $7(2)$ & $1(1)$ & $2(1)$ & 0.43 \\
\hline
\end{tabular}




\begin{tabular}{|c|c|c|c|c|}
\hline $\begin{array}{l}\text { Presence of total anomalous pulmonary } \\
\text { venous connection: } n(\%)\end{array}$ & $6(2)$ & $2(2)$ & $4(2)$ & 0.98 \\
\hline $\begin{array}{l}\text { Presence of partial anomalous pulmonary } \\
\text { venous connection: } n(\%)\end{array}$ & $4(1)$ & $0(0)$ & $4(2)$ & 0.15 \\
\hline \multicolumn{5}{|l|}{ Prior to the Norwood procedure: } \\
\hline Highest lactate (mmol/L) & $3.1(2.1-4.0)$ & $3.0(2.1-4.0)$ & $3.2(2.4-4.3)$ & 0.15 \\
\hline Intubated: n (\%) & $166(46)$ & $52(42)$ & $114(47)$ & 0.36 \\
\hline Inhaled nitric gases: n (\%) & $40(11)$ & $16(13)$ & $24(10)$ & 0.37 \\
\hline ECMO pre-Norwood: $n(\%)$ & $13(4)$ & $9(7)$ & $4(2)$ & 0.01 \\
\hline Any complications: $\mathrm{n}(\%)$ & $97(27)$ & $33(27)$ & $64(26)$ & 0.93 \\
\hline $\begin{array}{l}\text { Cardiac complications (RV dysfunction, } \\
\text { arrhythmia, etc.) }\end{array}$ & $38(10)$ & $13(11)$ & $25(10)$ & 0.94 \\
\hline $\begin{array}{l}\text { Respiratory complications (pleural } \\
\text { effusion, pneumothorax, etc.) }\end{array}$ & $26(7)$ & $5(4)$ & $21(9)$ & 0.11 \\
\hline $\begin{array}{l}\text { Neurological complications (stroke, bleed, } \\
\text { seizure, etc.) }\end{array}$ & $12(3)$ & $6(5)$ & $6(2)$ & 0.22 \\
\hline $\begin{array}{l}\text { Gl complications (necrotizing enterocolitis, } \\
\text { liver failure) }\end{array}$ & $15(4)$ & $4(3)$ & $11(5)$ & 0.56 \\
\hline $\begin{array}{l}\text { ID complication (sepsis, respiratory } \\
\text { infection, UTI, etc.) }\end{array}$ & $28(8)$ & $8(7)$ & $20(8)$ & 0.55 \\
\hline $\begin{array}{l}\text { Renal complication (acute or chronic renal } \\
\text { failure) }\end{array}$ & $12(3)$ & $4(3)$ & $8(3)$ & 0.98 \\
\hline Other complication (hematological, etc.) & $30(8)$ & $5(4)$ & $25(10)$ & 0.04 \\
\hline Randomized to modified BT shunt: $\mathrm{n}(\%)$ & $168(46)$ & $83(67)$ & $85(35)$ & $<0.001$ \\
\hline \multicolumn{5}{|l|}{ Norwood hospitalisation: } \\
\hline Year of Norwood: $n(\%)$ & & & & $<0.001$ \\
\hline 2005 & $84(23)$ & $17(14)$ & $67(28)$ & \\
\hline 2006 & $116(32)$ & $33(27)$ & $83(34)$ & \\
\hline 2007 & $113(31)$ & $43(35)$ & $70(29)$ & \\
\hline 2008 & $52(14)$ & $30(24)$ & $22(9)$ & \\
\hline Age at Norwood (days): median (IQR) & $6(4-8)$ & $6(4-8)$ & $6(4-8)$ & 0.33 \\
\hline Length of ICU stay (days): median (IQR) & $14(10-27)$ & $13(9-26)$ & $15(10-27)$ & 0.19 \\
\hline Length of stay (days): median (IQR) & $24(16-42)$ & $24(15-40)$ & $25(16-42)$ & 0.88 \\
\hline $\begin{array}{l}\text { Modified BT shunt at end of procedure: } \mathrm{n} \\
(\%)\end{array}$ & $168(46)$ & $83(67)$ & $85(35)$ & $<0.001$ \\
\hline $\begin{array}{l}\text { Diameter of modified BT shunt (mm) } \\
(\mathrm{n}=168) \text { : median (IQR) }\end{array}$ & $3.5(3.5-4.0)$ & $3.5(3.5-4.0)$ & $3.5(3.5-4.0)$ & 0.59 \\
\hline $\begin{array}{l}\text { Length of the modified BT shunt (mm) } \\
(n=168) \text { : median (IQR) }\end{array}$ & $1.7(1.2-2.35)$ & $1.8(1.3-2.5)$ & $1.7(1.5-2.0)$ & 0.86 \\
\hline $\begin{array}{l}\text { Diameter of the RVPA conduit }(\mathrm{mm}) \\
(\mathrm{n}=197) \text { : } \text { median (IQR) }\end{array}$ & $5.0(5.0-6.0)$ & $5.0(5.0-6.0)$ & $5.0(5.0-6.0)$ & 0.11 \\
\hline $\begin{array}{l}\text { Length of RVPA conduit (mm) ( } \mathrm{n}-197) \text { : } \\
\text { median (IQR) }\end{array}$ & $4.0(3.0-4.5)$ & $4.0(3.0-4.5)$ & $4.0(3.0-4.5)$ & 0.48 \\
\hline
\end{tabular}




\begin{tabular}{|c|c|c|c|c|}
\hline Total & $52(39-65)$ & $47(36-62)$ & $54(40-66)$ & 0.03 \\
\hline Bypass time (min): median (IQR) & $129(101-169)$ & $126(104-171)$ & $131(100-166)$ & 0.94 \\
\hline $\begin{array}{l}\text { Deep hypothermic circulatory arrest time } \\
\text { was used: } \mathrm{n}(\%)\end{array}$ & $344(94)$ & $116(94)$ & $228(94)$ & 0.97 \\
\hline $\begin{array}{l}\text { Deep hypothermic circulatory arrest time } \\
\text { (min): median (IQR) }\end{array}$ & $36(15-48)$ & $36(18-46)$ & $35(14-49)$ & 0.64 \\
\hline Regional cerebral perfusion used: $n(\%)$ & $152(42)$ & $47(38)$ & $105(43)$ & 0.34 \\
\hline $\begin{array}{l}\text { Regional cerebral perfusion time (min): } \\
\text { median (IQR) }\end{array}$ & $52(36-64)$ & $48(36-61)$ & $54(38-68)$ & 0.15 \\
\hline $\begin{array}{l}\text { Regional cerebral perfusion flow } \\
\text { (cc/kg/min): median (IQR) }\end{array}$ & $40(29-52)$ & $42(27-54)$ & $40(30-50)$ & 0.42 \\
\hline $\begin{array}{l}\text { Lowest temperature recorded during } \\
\text { Norwood (Celsius): median (IQR) }\end{array}$ & $17.6(16.1-18.0)$ & $17.6(16.2-18.0)$ & $17.6(16.1-18.0)$ & 1.00 \\
\hline $\begin{array}{l}\text { Lowest hematocrit recorded during } \\
\text { Norwood (\%): median (IQR) }\end{array}$ & $29(27-32)$ & $30(28-33)$ & $29(27-32)$ & 0.18 \\
\hline Ultrafiltration used during Norwood: $\mathrm{n}(\%)$ & $307(84)$ & $109(89)$ & $196(81)$ & 0.09 \\
\hline Ultrafiltration used during bypass: $\mathrm{n}(\%)$ & $240(78)$ & $86(70)$ & $154(64)$ & 0.82 \\
\hline Ultrafiltration used after bypass: $n(\%)$ & $253(82)$ & $89(72)$ & $164(67)$ & 0.80 \\
\hline Steroids given prior to Norwood: $\mathrm{n}(\%)$ & $321(88)$ & $115(93)$ & $206(85)$ & 0.02 \\
\hline Aprotinin was given: $\mathrm{n}(\%)$ & $284(78)$ & $86(70)$ & $196(81)$ & 0.01 \\
\hline Alpha blockade was given: $\mathrm{n}(\%)$ & $164(45)$ & $63(51)$ & $101(42)$ & 0.09 \\
\hline Type of alpha blockade: $n$ (\%) & & & & 0.06 \\
\hline Phenozybenzamine & $45(27)$ & $12(10)$ & $33(14)$ & \\
\hline Phentolamine & $119(73)$ & $51(41)$ & $68(28)$ & \\
\hline Coarctectomy: n (\%) & $114(31)$ & $37(30)$ & $77(32)$ & 0.70 \\
\hline Patch repair of aortic arch: $\mathrm{n}(\%)$ & $359(99)$ & $121(98)$ & $238(98)$ & 0.49 \\
\hline $\begin{array}{l}\text { Other procedure done at time of } \\
\text { Norwood: } \mathrm{n}(\%)\end{array}$ & $37(10)$ & $14(11)$ & $23(10)$ & 0.57 \\
\hline ECMO post-Norwood: $n(\%)$ & $23(6)$ & $10(8)$ & $13(5)$ & 0.31 \\
\hline CPR post-Norwood: $\mathrm{n}(\%)$ & $42(12)$ & $22(18)$ & $20(8)$ & 0.007 \\
\hline Age at extubation: median (IQR) & $12(9-16)$ & $12(9-16)$ & $11(9-15)$ & 0.17 \\
\hline $\begin{array}{l}\text { Duration of ventilation (days): median } \\
\text { (IQR) }\end{array}$ & $7(5-12)$ & $7(5-13)$ & $6(4-11)$ & 0.31 \\
\hline $\begin{array}{l}\text { Open sternum after leaving the operating } \\
\text { room: } n(\%)\end{array}$ & $267(73)$ & $96(78)$ & $171(71)$ & 0.13 \\
\hline Any complications post-Norwood: $n(\%)$ & $284(78)$ & $97(79)$ & $187(77)$ & 0.73 \\
\hline $\begin{array}{l}\text { Number of post-Norwood complications: } \\
\mathrm{n}(\%)\end{array}$ & $2(1-4)$ & $2(1-4)$ & $2(1-4)$ & 0.53 \\
\hline \multicolumn{5}{|l|}{ Post-Norwood complication: n (\%) } \\
\hline $\begin{array}{l}\text { Cardiac complications (RV dysfunction, } \\
\text { arrhythmia, etc.) }\end{array}$ & $136(37)$ & $56(46)$ & $80(33)$ & 0.02 \\
\hline $\begin{array}{l}\text { Respiratory complications (pleural } \\
\text { effusion, pneumothorax, etc.) }\end{array}$ & $161(44)$ & $53(43)$ & $108(44)$ & 0.78 \\
\hline
\end{tabular}




\begin{tabular}{|c|c|c|c|c|}
\hline $\begin{array}{l}\text { Neurological complications (stroke, bleed, } \\
\text { seizure, etc.) }\end{array}$ & $17(5)$ & $7(6)$ & $10(4)$ & 0.50 \\
\hline $\begin{array}{l}\text { GI complications (necrotizing enterocolitis, } \\
\text { liver failure) }\end{array}$ & $85(23)$ & $29(24)$ & $56(23)$ & 0.93 \\
\hline $\begin{array}{l}\text { ID complication (sepsis, repertory } \\
\text { infection, UTI, etc.) }\end{array}$ & $150(41)$ & $50(41)$ & $100(41)$ & 0.90 \\
\hline $\begin{array}{l}\text { Renal complication (acute or chronic renal } \\
\text { failure) }\end{array}$ & $24(7)$ & $11(9)$ & $13(5)$ & 0.19 \\
\hline Other complication (hematological, etc.) & $102(28)$ & $40(33)$ & $62(26)$ & 0.16 \\
\hline $\begin{array}{l}\text { Discharge home pre-stage } 2 \text { without } \\
\text { receiving a heart transplant: } \mathrm{n}(\%)\end{array}$ & $353(97)$ & $118(96)$ & $235(97)$ & 0.55 \\
\hline $\begin{array}{l}\text { Oxygen saturation at discharge: median } \\
\text { (IQR) }\end{array}$ & $82.0(80.0-85.0)$ & $81.5(79.0-85.0)$ & $83.0(80.0-85.0)$ & 0.23 \\
\hline Oxygen at discharge: $\mathrm{n}(\%)$ & $39(11)$ & $9(7)$ & $30(12)$ & 0.14 \\
\hline $\begin{array}{l}\text { Number of medications at discharge: } \\
\text { median (IQR) }\end{array}$ & $5(4-6)$ & $5(4-7)$ & $5(4-6)$ & 0.61 \\
\hline Discharged on an ACE-inhibitor: $\mathrm{n}(\%)$ & $136(37)$ & $49(40)$ & $87(36)$ & 0.47 \\
\hline Discharged on a Beta-blocker: $\mathrm{n}(\%)$ & $9(2)$ & $2(2)$ & $7(3)$ & 0.46 \\
\hline Discharged on Inotrope: $\mathrm{n}(\%)$ & $10(3)$ & $6(5)$ & $4(2)$ & 0.07 \\
\hline Discharged on a diuretic: $\mathrm{n}(\%)$ & $306(84)$ & $102(83)$ & $204(84)$ & 0.74 \\
\hline Discharged with an anti-arrhythmic: $\mathrm{n}(\%)$ & $12(3)$ & $4(3)$ & $8(3)$ & 0.98 \\
\hline $\begin{array}{l}\text { Discharged on anti-hypertensive } \\
\text { medication: } \mathrm{n}(\%)\end{array}$ & $15(4)$ & $5(4)$ & $10(4)$ & 0.98 \\
\hline Able to proceed to stage II: $n(\%)$ & $315(86)$ & $100(81)$ & $215(88)$ & 0.05 \\
\hline Died without achieving stage II: $\mathrm{n}(\%)$ & $48(13)$ & $21(17)$ & $26(11)$ & 0.06 \\
\hline $\begin{array}{l}\text { Received a heart transplant without } \\
\text { achieving stage II: } \mathrm{n}(\%)\end{array}$ & $3(1)$ & $2(2)$ & $1(0.5)$ & 0.23 \\
\hline
\end{tabular}

Abbreviations: RVEF, right ventricular ejection fraction; RVAFC, right ventricular fractional area of change; n, number; g, gram; SD, standard deviation; IQR, interquartile range; HLHS, hypoplastic left heart syndrome; RV, right ventricle; AVSD, atrioventricular septal defect; LV, left ventricle; mm, milimeter; mmol/L, millimole per litre; ECMO, extracorporeal membrane oxygenation; GI, gastrointestinal; ID, infectious disease; UTI, urinary tract infection; BT, Blalock-Taussig; ICU, intensive care unit; RVPA, right ventricle to pulmonary artery; min, minute; cc/kg/min, milliliter per kilogram per min; CPR, cardiopulmonary resuscitation; ACE, angiotensin-converting enzyme. 
Table 3. Comparison of baseline echocardiographic measurements between the ventricular dysfunction and the normal function cohorts.

\begin{tabular}{|c|c|c|c|c|}
\hline & $\begin{array}{l}\text { All patients } \\
\text { with RVEF or } \\
\text { RVFAC } \\
\text { measurements } \\
\text { (n=365) }\end{array}$ & $\begin{array}{l}\text { Ventricular } \\
\text { dysfunction } \\
\text { cohort ( } n=123)\end{array}$ & $\begin{array}{l}\text { Normal } \\
\text { function cohort } \\
(n=242)\end{array}$ & P-value \\
\hline \multicolumn{5}{|l|}{ Baseline echocardiogram } \\
\hline \multicolumn{5}{|l|}{ Anatomical information: } \\
\hline Patent mitral valve: $\mathrm{n}(\%)$ & $185(51)$ & $60(49)$ & $125(52)$ & 0.63 \\
\hline Patent aortic valve: $\mathrm{n}(\%)$ & $155(42)$ & $49(32)$ & $106(44)$ & 0.18 \\
\hline Left ventricle present: $n(\%)$ & $256(70)$ & $85(69)$ & $171(71)$ & 0.86 \\
\hline $\begin{array}{l}\text { Native ascending aorta dimension }(\mathrm{cm}) \text { : } \\
\text { median (IQR) }\end{array}$ & $0.3(0.2-0.5)$ & $0.3(0.2-0.5)$ & $0.3(0.2-0.5)$ & 0.70 \\
\hline LPA dimension (cm): median (IQR) & $0.40(0.34-0.47)$ & $0.41(0.35-0.47)$ & $0.40(0.34-0.47)$ & 0.55 \\
\hline $\begin{array}{l}\text { ASD Doppler gradient }(\mathrm{mmHg}): \text { median } \\
\text { (IQR) }\end{array}$ & $4.8(2.3-7.6)$ & $5.0(2.1-8.2)$ & $4.8(2.3-7.4)$ & 0.94 \\
\hline \multicolumn{5}{|l|}{ RV and TV measurements: } \\
\hline $\begin{array}{l}\text { RV end-diastolic long axis dimension }(\mathrm{cm}) \text { : } \\
\text { median (IQR) }\end{array}$ & $3.0(2.7-3.2)$ & $3.0(2.8-3.2)$ & $3.0(2.7-3.2)$ & 0.18 \\
\hline $\begin{array}{l}\text { RV end-diastolic short axis dimension }(\mathrm{cm}) \text { : } \\
\text { median (IQR) }\end{array}$ & $2.2(1.9-2.7)$ & $2.4(2.0-2.7)$ & $2.2(1.9-3.0)$ & 0.01 \\
\hline RVEF (\%): median (IQR) & $46.6(41.3-52.8)$ & $44.5(39.8-48.5)$ & $47.6(41.6-53.8)$ & 0.002 \\
\hline RVFAC (\%): median (IQR) & $35.0(30.0-40.0)$ & $33.0(28.0-37.0)$ & $36.0(31.0-42.0)$ & $<0.001$ \\
\hline RV eccentricity: median (IQR) & $1.31(1.08-1.58)$ & $1.27(1.08-1.50)$ & $1.35(1.07-1.64)$ & 0.09 \\
\hline $\mathrm{RV}$ isovolumic time (msec): median (IQR) & $\begin{array}{l}67.0(34.0- \\
110.0)\end{array}$ & $\begin{array}{l}66.0(34.0- \\
104.0)\end{array}$ & $\begin{array}{l}67.5(34.0- \\
110.0)\end{array}$ & 0.92 \\
\hline $\begin{array}{l}\mathrm{RV} \text { indexed end-diastolic volume } \\
\left(\mathrm{mL}^{3} / \mathrm{BSA}^{*} 1.3\right) \text { : median (IQR) }\end{array}$ & $83.7(69.7-99.5)$ & $\begin{array}{l}92.5(76.2- \\
107.4)\end{array}$ & $80.4(68.8-94.9)$ & 0.008 \\
\hline $\begin{array}{l}\text { RV indexed end-diastolic area } \\
\left(\mathrm{mL}^{3} / \mathrm{BSA}^{*} 1.3\right): \text { median (IQR) }\end{array}$ & $20.9(17.9-24.0)$ & $22.4(19.9-25.4)$ & $20.2(17.5-23.50$ & 0.0001 \\
\hline $\begin{array}{l}\text { Tissue Doppler: RV peak systolic velocity } \\
\text { (mmHg): median (IQR) }\end{array}$ & $8.0(6.2-9.1)$ & $7.3(6.0-9.4)$ & $8.0(6.8-9.0)$ & 0.44 \\
\hline $\begin{array}{l}\text { Anteroposterior TV annulus }(\mathrm{cm}) \text { : median } \\
\text { (IQR) }\end{array}$ & $1.22(1.09-1.37)$ & $1.22(1.11-1.39)$ & $1.22(1.08-1.36)$ & 0.24 \\
\hline $\begin{array}{l}\text { Anteroposterior TV annulus z score: median } \\
\text { (IQR) }\end{array}$ & $1.7(0.9-2.6)$ & $1.7(0.8-2.9)$ & $1.7(1.0-2.6)$ & 0.98 \\
\hline Transverse TV annulus (cm): median (IQR) & $1.31(1.17-1.49)$ & $1.34(1.19-1.52)$ & $1.31(1.15-1.48)$ & 0.23 \\
\hline Transverse TV annulus z score: median (IQR) & $1.6(1.0-2.6)$ & $1.7(1.0-2.6)$ & $1.6(1.0-2.6)$ & 0.55 \\
\hline TV annulus area $\left(\mathrm{mm}^{2}\right)$ : median (IQR) & $124.6(101.1-$ & $129.2(107.3-$ & $122.8(99.7-$ & 0.13 \\
\hline
\end{tabular}




\begin{tabular}{|c|c|c|c|c|}
\hline & 152.1) & 161.7) & 150.7) & \\
\hline $\begin{array}{l}\text { TV indexed annular area }\left(\mathrm{mm}^{2} / \mathrm{BSA}\right) \text { : } \\
\text { median (IQR) }\end{array}$ & $6.1(4.9-7.2)$ & $6.1(5.1-7.5)$ & $6.0(4.8-7.1)$ & 0.16 \\
\hline TV annular area z score: median (IQR) & $2.5(1.2-3.7)$ & $2.6(1.3-4.4)$ & $2.5(1.1-3.6)$ & 0.16 \\
\hline TV regurgitation: $\mathrm{n}(\%)$ & $253(71)$ & $91(74)$ & $162(67)$ & 0.20 \\
\hline TV regurgitation severity $(n=253): n(\%)$ & & & & 0.79 \\
\hline Mild & $211(83)$ & $74(60)$ & $137(57)$ & \\
\hline Moderate & $35(14)$ & $14(11)$ & $21(9)$ & \\
\hline Severe & $7(3)$ & $3(2)$ & $4(2)$ & \\
\hline \multicolumn{5}{|l|}{ Outflow tracts: } \\
\hline Aortic valve regurgitation: $\mathrm{n}(\%)$ & $19(12)$ & $7(6)$ & $12(5)$ & 0.60 \\
\hline $\begin{array}{l}\text { Aortic valve regurgitation severity }(n=19): n \\
(\%)\end{array}$ & & & & 0.25 \\
\hline Mild & $17(89)$ & $7(6)$ & $10(4)$ & \\
\hline Moderate & $2(11)$ & $0(0)$ & $2(1)$ & \\
\hline Severe & $0(0)$ & $0(0)$ & $0(0)$ & \\
\hline Neoaortic valve regurgitation: $\mathrm{n}(\%)$ & $85(24)$ & $34(28)$ & $51(21)$ & 0.15 \\
\hline $\begin{array}{l}\text { Neoaortic valve regurgitation severity } \\
(n=85): n(\%)\end{array}$ & & & & 0.22 \\
\hline Mild & $84(97)$ & $33(27)$ & $51(21)$ & \\
\hline Moderate & $1(3)$ & $1(1)$ & $0(0)$ & \\
\hline Severe & $0(0)$ & $0(0)$ & $0(0)$ & \\
\hline \multicolumn{5}{|l|}{ LV measurements: } \\
\hline $\begin{array}{l}\text { LV end-diastolic long axis dimension (cm): } \\
\text { median (IQR) }\end{array}$ & $1.5(1.1-2.0)$ & $1.4(1.0-1.8)$ & $1.5(1.2-2.0)$ & 0.20 \\
\hline LV end-diastolic volume (mL): median (IQR) & $0.7(0.3-1.4)$ & $0.5(0.3-1.2)$ & $0.7(0.3-1.5)$ & 0.21 \\
\hline LV end-diastolic volume (mL): median (IQR) & $0.5(0.2-0.9)$ & $0.5(0.2-0.8)$ & $0.5(0.2-1.0)$ & 0.24 \\
\hline LV ventricular mass (g): median (IQR) & $3.0(2.0-4.2)$ & $2.6(1.7-4.0)$ & $3.0(2.0-4.4)$ & 0.48 \\
\hline LV ejection fraction (\%): median (IQR) & $25.0(9.5-44.1)$ & $26.0(8.8-44.2)$ & $23.8(10.0-44.0)$ & 0.89 \\
\hline Mitral valve regurgitation: $\mathrm{n}(\%)$ & $85(46)$ & $23(19)$ & $62(26)$ & 0.18 \\
\hline $\begin{array}{l}\text { Mitral valve regurgitation severity }(n=85): n \\
(\%)\end{array}$ & & & & 0.48 \\
\hline Mild & $76(89)$ & $22(18)$ & $54(22)$ & \\
\hline Moderate & $7(8)$ & $1(1)$ & $6(2)$ & \\
\hline Severe & $2(2)$ & $0(0)$ & $2(1)$ & \\
\hline
\end{tabular}

Abbreviations: RVEF, right ventricular ejection fraction; RVAFC, right ventricular fractional area of change; n, number; cm, centimeter; IQR, interquartile range; LPA, left pulmonary artery; ASD, atrial septal defect; RV, right ventricle; TV, tricuspid valve; BSA, body surface area; mm, millimeter; msec, millisecond; mmHg, millimeter of mercury; LV, left ventricle; $\mathrm{mL}$, milliliter; g, gram. 


\section{Table 4. Comparison of post-Norwood echocardiographic measurements between the ventricular dysfunction and the normal function cohorts.}

\begin{tabular}{|c|c|c|c|c|}
\hline & $\begin{array}{l}\text { All patients } \\
\text { with RVEF or } \\
\text { RVFAC } \\
\text { measurements } \\
(\mathrm{n}=365)\end{array}$ & $\begin{array}{l}\text { Ventricular } \\
\text { dysfunction } \\
\text { cohort ( } n=123)\end{array}$ & $\begin{array}{l}\text { Normal } \\
\text { function cohort } \\
\text { ( } n=242)\end{array}$ & P-value \\
\hline \multicolumn{5}{|l|}{ Post-Norwood echocardiogram } \\
\hline \multicolumn{5}{|l|}{ Anatomical information: } \\
\hline $\begin{array}{l}\text { Narrowest distal arch diameter }(\mathrm{cm}) \text { : } \\
\text { median (IQR) }\end{array}$ & $0.62(0.51-0.73)$ & $0.60(0.51-0.71)$ & $0.63(0.51-0.74)$ & 0.48 \\
\hline $\begin{array}{l}\text { ASD Doppler gradient (mmHg): median } \\
\text { (IQR) }\end{array}$ & $4.8(2.3-7.6)$ & $5.0(2.1-8.2)$ & $4.8(2.3-7.4)$ & 0.94 \\
\hline \multicolumn{5}{|l|}{ RV and TV measurements: } \\
\hline $\begin{array}{l}\text { Anteroposterior TV annulus (cm): median } \\
\text { (IQR) }\end{array}$ & $1.33(1.17-1.48)$ & $1.36(1.2-1.56)$ & $1.29(1.16-1.44)$ & 0.005 \\
\hline $\begin{array}{l}\text { Anteroposterior TV annulus z score: } \\
\text { median (IQR) }\end{array}$ & $2.1(1.2-3.2)$ & $2.4(1.5-3.6)$ & $2.0(1.1-3.1)$ & 0.005 \\
\hline Transverse TV annulus (cm): median (IQR) & $1.41(1.25-1.59)$ & $1.45(1.30-1.60)$ & $1.38(1.22-1.57)$ & 0.008 \\
\hline $\begin{array}{l}\text { Transverse TV annulus z score: median } \\
\text { (IQR) }\end{array}$ & $2.0(1.2-2.9)$ & $2.2(1.3-3.0)$ & $1.9(1.1-3.1)$ & 0.13 \\
\hline TV annulus area $\left(\mathrm{mm}^{2}\right)$ : median (IQR) & $\begin{array}{l}148.7(117.7- \\
174.7)\end{array}$ & $\begin{array}{l}159.4(127.2- \\
185.0)\end{array}$ & $\begin{array}{l}144.1(113.9- \\
170.6)\end{array}$ & 0.002 \\
\hline $\begin{array}{l}\text { TV indexed annular area }\left(\mathrm{mm}^{2} / \mathrm{BSA}\right) \text { : } \\
\text { median (IQR) }\end{array}$ & $6.7(5.6-8.2)$ & $7.3(6.3-8.6)$ & $6.7(5.3-8.0)$ & 0.002 \\
\hline TV annular area $z$ score: median (IQR) & $3.4(2.0-4.7)$ & $7.3(6.3-8.6)$ & $3.2(1.8-4.5)$ & 0.001 \\
\hline TV regurgitation: $\mathrm{n}(\%)$ & $325(89)$ & $110(89)$ & $215(89)$ & 0.87 \\
\hline TV regurgitation severity $(n=325): n(\%)$ & & & & 0.13 \\
\hline Mild & $246(78)$ & $78(63)$ & $168(69)$ & \\
\hline Moderate & $69(21)$ & $26(21)$ & $6(2)$ & \\
\hline Severe & $10(3)$ & $6(5)$ & $4(2)$ & \\
\hline Worsening TV regurgitation: $\mathrm{n}(\%)$ & $129(35)$ & $44(36)$ & $85(35)$ & 0.99 \\
\hline RVEF (\%): median (IQR) & $46.8(41.4-52.5)$ & $39.2(35.8-42.2)$ & $50.0(46.8-54.2)$ & $<0.0001$ \\
\hline Worsening RVEF: $\mathrm{n}(\%)$ & $123(34)$ & $61(50)$ & $62(26)$ & $<0.0001$ \\
\hline RVFAC (\%): median (IQR) & $36.0(32.0-41.0)$ & $30.0(26.0-33.0)$ & $39.0(36.0-40.0)$ & $<0.0001$ \\
\hline Worsening RVFAC: $\mathrm{n}(\%)$ & $144(39)$ & $76(62)$ & $68(28)$ & $<0.0001$ \\
\hline RV eccentricity: median (IQR) & $1.24(1.04-1.49)$ & $1.22(1.00-1.45)$ & $1.25(1.06-1.51)$ & 0.09 \\
\hline $\mathrm{RV}$ isovolumic time (msec): median (IQR) & $84.0(62.0-$ & $79.5(60.0-$ & $85.5(62.5-$ & 0.07 \\
\hline
\end{tabular}




\begin{tabular}{|c|c|c|c|c|}
\hline & 109.0) & 103.0) & 112.5) & \\
\hline $\begin{array}{l}\text { RV indexed end-diastolic volume } \\
\left(\mathrm{mL}^{3} / \mathrm{BSA}^{*} 1.3\right) \text { : median (IQR) }\end{array}$ & \begin{tabular}{|l|}
$89.0(74.5-$ \\
$105.0)$ \\
\end{tabular} & \begin{tabular}{|l|}
$99.6(83.3-$ \\
$118.1)$ \\
\end{tabular} & 84.6 (69.2-99.4) & $<0.0001$ \\
\hline $\begin{array}{l}\text { RV indexed end-diastolic area } \\
\left(\mathrm{mL}^{3} / \mathrm{BSA}^{*} 1.3\right): \text { median (IQR) }\end{array}$ & $21.8(18.6-24.9)$ & $23.3(20.7-26.9)$ & $20.9(17.8-24.1)$ & $<0.0001$ \\
\hline $\begin{array}{l}\text { Tissue Doppler: RV peak systolic velocity: } \\
\text { median (mmHg): median (IQR) }\end{array}$ & $4.1(3.7-5.0)$ & $4.0(3.5-5.0)$ & $4.1(3.7-5.0)$ & 0.57 \\
\hline \multicolumn{5}{|l|}{ Outflow tracts: } \\
\hline Aortic valve regurgitation: $\mathrm{n}(\%)$ & $17(14)$ & $6(5)$ & $11(5)$ & 0.68 \\
\hline $\begin{array}{l}\text { Aortic valve regurgitation severity }(n=17) \text { : } \\
n(\%)\end{array}$ & & & & 0.45 \\
\hline Mild & $16(94)$ & $6(5)$ & $10(4)$ & \\
\hline Moderate & $1(6)$ & $0(0)$ & $1(0.5)$ & \\
\hline Severe & $0(0)$ & $0(0)$ & $0(0)$ & \\
\hline Neoaortic valve regurgitation: $\mathrm{n}(\%)$ & $176(48)$ & $59(48)$ & $117(48)$ & 0.95 \\
\hline $\begin{array}{l}\text { Neoaortic valve regurgitation severity } \\
(n=176): n(\%)\end{array}$ & & & & 0.26 \\
\hline Mild & $165(94)$ & $54(44)$ & $111(46)$ & \\
\hline Moderate & $10(6)$ & $5(4)$ & $5(2)$ & \\
\hline Severe & $0(0)$ & $0(0)$ & $0(0)$ & \\
\hline \multicolumn{5}{|l|}{ LV measurements: } \\
\hline Mitral valve regurgitation: $\mathrm{n}(\%)$ & $96(59)$ & $28(23)$ & $68(28)$ & 0.57 \\
\hline $\begin{array}{l}\text { Mitral valve regurgitation severity }(\mathrm{n}=96) \text { : } \\
\mathrm{n}(\%)\end{array}$ & & & & 0.82 \\
\hline Mild & $81(84)$ & $24(20)$ & $57(24)$ & \\
\hline Moderate & $15(16)$ & $4(3)$ & $11(5)$ & \\
\hline Severe & $0(0)$ & $0(0)$ & $0(0)$ & \\
\hline LV ejection fraction (\%): median (IQR) & $19.2(5.7-35.7)$ & $20.0(6.3-35.3)$ & $17.6(5.6-36.8)$ & 0.72 \\
\hline
\end{tabular}

Abbreviations: RVEF, right ventricular ejection fraction; RVAFC, right ventricular fractional area of change; n, number; cm, centimeter; IQR, interquartile range; ASD, atrial septal defect; $\mathrm{RV}$, right ventricle; TV, tricuspid valve; $\mathrm{mm}$, millimeter; $\mathrm{BSA}$, body surface area; $\mathrm{mL}$, milliliter; m msec, millisecond; mmHg, millimeter of mercury; LV, left ventricle; 


\section{Table 5. Independent risk factors for death or heart transplant without achieving the stage II procedure}

\begin{tabular}{|l|c|c|c|}
\hline Risk factors & $\begin{array}{c}\text { Parameter } \\
\text { estimate }\end{array}$ & $\mathbf{9 5 \%} \mathbf{C l}$ & P value \\
\hline MBTS vs. RVPA conduit & 2.22 & $0.19-4.24$ & 0.03 \\
\hline Post-Norwood infectious disease complication & 1.51 & $0.62-2.40$ & $<0.001$ \\
\hline Surgeon volume of 5 Norwoods per year & 1.10 & $0.24-1.97$ & 0.01 \\
\hline RV end-diastolic long axis dimension per cm & 1.05 & $0.02-3.1$ & 0.05 \\
\hline
\end{tabular}

Abbreviations: MBTS, modified Blalock-Taussig shunt; RVPA, right ventricle to pulmonary artery; RV, right ventricle; cm, centrimeters. 


\section{References}

Abe, T. et al., 2013. Clinical characteristics and long-term outcome of acute myocarditis in children. Heart and Vessels, 28(5), pp.632-638.

Albers, E.L., Bichell, D.P. \& McLaughlin, B., 2010. New approaches to neuroprotection in infant heart surgery. Pediatric Research, 68(1), pp.1-9.

Alexander, P.M.A. et al., 2013. Long-Term Outcomes of Dilated Cardiomyopathy Diagnosed During Childhood: Results From a National Population-Based Study of Childhood Cardiomyopathy. Circulation, 128(18), pp.2039-2046.

Algra, S.O. et al., 2012. Bedside prediction rule for infections after pediatric cardiac surgery. Intensive care medicine, 38(3), pp.474-481.

Alsoufi, B. et al., 2015. Impact of Patient Characteristics and Anatomy on Results of Norwood Operation for Hypoplastic Left Heart Syndrome. The Annals of thoracic surgery, 100(2), pp.591-598.

Altmann, K. et al., 2000. Two-dimensional echocardiographic assessment of right ventricular function as a predictor of outcome in hypoplastic left heart syndrome. The American Journal of Cardiology, 86(9), pp.964-968.

Anderson, B.R. et al., 2016. The Norwood operation: Relative effects of surgeon and institutional volumes on outcomes and resource utilization. Cardiology in the young, 26(4), pp.683-692.

Atz, A.M. et al., 2011. Factors associated with serum brain natriuretic peptide levels after the Fontan procedure. Congenital heart disease, pp.1-9.

Atz, A.M. et al., 2010. Prenatal diagnosis and risk factors for preoperative death in neonates with single right ventricle and systemic outflow obstruction: Screening data from the Pediatric Heart Network Single Ventricle Reconstruction Trial*. The Journal of Thoracic and Cardiovascular Surgery, 140(6), pp.1245-1250. 
Austin, P.C. \& Tu, J.V., 2004. Bootstrap Methods for Developing Predictive Models Taylor \& Francis, Ltd, ed. The American Statistician, 58(2), pp.131-137.

Baird, R.J. \& Adiseshiah, M., 1976. The response of diastolic myocardial tissue pressure and regional coronary blood flow to increased preload from blood, colloid, crystalloid. Surgery, 79(6), pp.644-651.

Barker, G.M. et al., 2010. Major infection after pediatric cardiac surgery: a risk estimation model. The Annals of thoracic surgery, 89(3), pp.843-850.

Bartram, U., Grünenfelder, J. \& Van Praagh, R., 1997. Causes of death after the modified Norwood procedure: a study of 122 postmortem cases. The Annals of thoracic surgery, 64(6), pp.1795-1802.

Bazzani, L.G. \& Marcin, J.P., 2007. Case volume and mortality in pediatric cardiac surgery patients in California, 1998-2003. Circulation, 115(20), pp.2652-2659.

Ben-Ami, E. et al., 2008. Risk factors for sternal wound infection in children undergoing cardiac surgery: a case-control study. The Journal of hospital infection, 70(4), pp.335-340.

Blackstone, E.H., Naftel, D.C. \& Turner JR, M.E., 1986. Incorporating. Journal of American Statistical Association, 81, pp.615-624.

Bradley, S.M. et al., 2004. Hemodynamic status after the Norwood procedure: a comparison of right ventricle-to-pulmonary artery connection versus modified Blalock-Taussig shunt. The Annals of thoracic surgery, 78(3), pp.933-41-discussion 933-41.

Bull, C. et al., 2000. Scientific, ethical, and logistical considerations in introducing a new operation: a retrospective cohort study from paediatric cardiac surgery. $B M J, 320(7243)$, pp.1168-1173.

Cain, K.C. et al., 2011. Bias due to left truncation and left censoring in longitudinal studies of developmental and disease processes. American journal of epidemiology, 173(9), pp.10781084.

Carlo, W.F. et al., 2016. Impact of initial Norwood shunt type on young hypoplastic left heart 
syndrome patients listed for heart transplant: A multi-institutional study. The Journal of heart and lung transplantation: the official publication of the International Society for Heart Transplantation, 35(3), pp.301-305.

Center for Disease Control and Prevention (CDC), 2007. Hospital Stays, Hospital Charges, and In-Hospital Deaths Among Infants with Selected Birth Defects --- United States, 2003. MMWR Morb Mortal WKLy Rep, 56(2), pp.25-29. Available at: https://www-cdcgov.myaccess.library.utoronto.ca/mmwr/preview/mmwrhtml/mm5602al.htm [Accessed September 30, 2016].

Checchia, P.A. et al., 2005. The effect of surgical case volume on outcome after the Norwood procedure. The Journal of Thoracic and Cardiovascular Surgery, 129(4), pp.754-759.

Chetan, D. et al., 2013. Surgical palliation strategy does not affect interstage ventricular dysfunction or atrioventricular valve regurgitation in children with hypoplastic left heart syndrome and variants. Circulation, 128(11 Suppl 1), pp.S205-12.

Clark, T.G. et al., 2003. Survival Analysis Part I: Basic concepts and first analyses. British Journal of Cancer, 89(2), pp.232-238.

Costello, J.M. et al., 2010. Risk factors for surgical site infection after cardiac surgery in children. The Annals of thoracic surgery, 89(6), pp.1833-41- discussion 1841-2.

Cross, R.R. et al., 2014. Identified mortality risk factors associated with presentation, initial hospitalisation, and interstage period for the Norwood operation in a multi-centre registry: a report from the national pediatric cardiology-quality improvement collaborative. Cardiology in the young, 24(2), pp.253-262.

Dai, C.-C. et al., 2013. Dyssynchronous ventricular contraction in Wolff-Parkinson-White syndrome: a risk factor for the development of dilated cardiomyopathy. European journal of pediatrics, 172(11), pp.1491-1500.

DeCampli, W.M. et al., 2013. The effect of epinephrine on coronary flow in the setting of a systemic-to-pulmonary artery shunt. World Journal for Pediatric and Congenital Heart Surgery, 4(4), pp.373-379. 
Donnelly, J.P. et al., 1998. Resting coronary flow and coronary flow reserve in human infants after repair or palliation of congenital heart defects as measured by positron emission tomography. The Journal of Thoracic and Cardiovascular Surgery, 115(1), pp.103-110.

Driscol T.E, Moir T.W. \& EcksTein R.W., Autoregulation of coronary blood flow: Effect of interarterial pressure gradients. Circulation Research, 15, pp.103-111.

Everitt, M.D. et al., 2012. Early survival after heart transplant in young infants is lowest after failed single-ventricle palliation: A multi-institutional study. The Journal of Heart and Lung Transplantation, 31(5), pp.509-516.

Everitt, M.D. et al., 2014. Recovery of Echocardiographic Function in Children With Idiopathic Dilated Cardiomyopathy. Journal of the American College of Cardiology, 63(14), pp.14051413.

Frommelt, P.C. et al., 2012. Does Initial Shunt Type for the Norwood Procedure Affect Echocardiographic Measures of Cardiac Size and Function During Infancy?: The Single Ventricle Reconstruction Trial. Circulation, 125(21), pp.2630-2638.

Furck, A.K. et al., 2010. Outcome of the Norwood operation in patients with hypoplastic left heart syndrome: a 12-year single-center survey. The Journal of Thoracic and Cardiovascular Surgery, 139(2), pp.359-365.

Ghanayem, N.S. et al., 2006. Right ventricle-to-pulmonary artery conduit versus Blalock-Taussig shunt: a hemodynamic comparison. The Annals of thoracic surgery, 82(5), pp.1603-9discussion 1609-10.

Ghanayem, N.S. et al, 2012 Interstage mortality after the Norwood procedure: Results of the multicenter Single Ventricle Reconstruction trial. The Journal of Thoracic and Cardiovascular Surgery, 144(4), pp.896-906.

Gjesdal, O., Bluemke, D.A. \& Lima, J.A., 2011. remoldling. Nature Reviews Cardiology, 8(12), pp.673-685.

Hehir, D.A. et al., 2008. Risk factors for interstage death after stage 1 reconstruction of hypoplastic left heart syndrome and variants. The Journal of Thoracic and Cardiovascular 
Surgery, 136(1), pp.94-9-99.e1-3.

Helbing, W.A. et al., 1995. Comparison of echocardiographic methods with magnetic resonance imaging for assessment of right ventricular function in children. The American Journal of Cardiology, 76(8), pp.589-594.

Henkens, I.R. et al., 2007. Predicting Outcome of Pulmonary Valve Replacement in Adult Tetralogy of Fallot Patients. The Annals of thoracic surgery, 83(3), pp.907-911.

Hill, G.D. et al., 2015. Impact of initial norwood shunt type on right ventricular deformation: the single ventricle reconstruction trial. Journal of the American Society of Echocardiography: official publication of the American Society of Echocardiography, 28(5), pp.517-521.

Holzmann-Pazgal, G. et al., 2008. Case-control study of pediatric cardiothoracic surgical site infections . Infection control and hospital epidemiology, 29(1), pp.76-79.

Honjo, O. et al., 2011. Atrioventricular valve repair in patients with functional single-ventricle physiology: impact of ventricular and valve function and morphology on survival and reintervention. The Journal of Thoracic and Cardiovascular Surgery, 142(2), pp.326-35.e2.

Hornik, C.P. et al., 2012. Relative impact of surgeon and center volume on early mortality after the Norwood operation. The Annals of thoracic surgery, 93(6), pp.1992-1997.

Hraska, V. et al., 2003. Is a learning curve for arterial switch operation in small countries still acceptable? Model for cooperation in Europe. European journal of cardio-thoracic surgery : official journal of the European Association for Cardio-thoracic Surgery, 24(3), pp.352357.

Hsia, T.-Y. et al., 2011. Use of mathematical modeling to compare and predict hemodynamic effects between hybrid and surgical Norwood palliations for hypoplastic left heart syndrome. Circulation, 124(11 Suppl), pp.S204-10.

Jean-St-Michel, E. et al., 2016. Outcomes in Patients with Persistent Ventricular Dysfunction After Stage I Palliation for Hypoplastic Left Heart Syndrome. Pediatric Cardiology, 37(2), pp.239-247. 
Jenkins, K.J. et al., 1995. In-hospital mortality for surgical repair of congenital heart defects: preliminary observations of variation by hospital caseload. PEDIATRICS, 95(3), pp.323330.

Karamlou, T. et al., 2012. Impact of Single-Ventricle Physiology on Death After Heart Transplantation in Adults With Congenital Heart Disease. The Annals of thoracic surgery, 94(4), pp.1281-1288.

Kulkarni, A. et al., 2016. Outcomes and risk factors for listing for heart transplantation after the Norwood procedure: An analysis of the Single Ventricle Reconstruction Trial. The Journal of heart and lung transplantation : the official publication of the International Society for Heart Transplantation, 35(3), pp.306-311.

Lancellotti, P. \& Moonen, M., 2012. Left ventricular dyssynchrony: a dynamic condition. Heart Failure Reviews, 17(6), pp.747-753.

Lang, R.M. et al., 2015. Recommendations for cardiac chamber quantification by echocardiography in adults: an update from the American Society of Echocardiography and the European Association of Cardiovascular Imaging. European Heart Journal Cardiovascular Imaging, 16(3), pp.233-270.

Larose, E. et al., 2007. Right Ventricular Dysfunction Assessed by Cardiovascular Magnetic Resonance Imaging Predicts Poor Prognosis Late After Myocardial Infarction. Journal of the American College of Cardiology, 49(8), pp.855-862.

Laskey, W.K. et al., 1984. Left ventricular mechanics in dilated cardiomyopathy. The American Journal of Cardiology, 54(6), pp.620-625.

Lawrence G Rudski MD, F.C. et al., 2010. Guidelines for the Echocardiographic Assessment ofthe Right Heart in Adults: A Report from the AmericanSociety of Echocardiography. Journal of the American Society of Echocardiography, 23(7), pp.685-713.

Ling, L.F. \& Marwick, T.H., 2012. Echocardiographic Assessment ofRight Ventricular Function. $J C M G, 5(7)$, pp.747-753.

Mahony, L. et al., 2006. The Pediatric Heart Network: a primer for the conduct of multicenter 
studies in children with congenital and acquired heart disease. Pediatric Cardiology, 27(2), pp.191-198.

Matsumura, Y. et al., 2013. Left ventricular reverse remodeling in long-term ( $>12$ years) survivors with idiopathic dilated cardiomyopathy. The American Journal of Cardiology, 111(1), pp.106-110.

Menon, S.C. et al., 2013. Effect of ventriculotomy on right-ventricular remodeling in hypoplastic left heart syndrome: a histopathological and echocardiography correlation study. Pediatric Cardiology, 34(2), pp.354-363.

Muthurangu, V., Simpson, J.M. \& Razavi, R.S., 2005. Spontaneous improvement of severe right ventricular dysfunction in the setting of hypoplasia of the left heart. Cardiology in the young, 15(1), pp.75-78.

Newburger, J.W. et al., 1993. A comparison of the perioperative neurologic effects of hypothermic circulatory arrest versus low-flow cardiopulmonary bypass in infant heart surgery. New England Journal of Medicine, pp.1-8.

Newburger, J.W. et al., 2014. Transplantation-free survival and interventions at 3 years in the single ventricle reconstruction trial. Circulation, 129(20), pp.2013-2020.

Noonan, J.A. \& Nadas, A.S., 1958. The hypoplastic left heart syndrome; an analysis of 101 cases. Pediatric Clinics of North America, 5(4), pp.1029-1056.

Norwood, W.I. et al., 1981. Experience with operations for hypoplastic left heart syndrome. $J$ Thorac Cardiovasc Surg, 82(4), pp.511-519.

O'Connor, M.J. et al., 2012. Outcomes of infants undergoing superior cavopulmonary connection in the presence of ventricular dysfunction. Pediatric Cardiology, 33(4), pp.547-553.

Ohye, R.G. et al., 2012. Cause, timing, and location of death in the Single Ventricle Reconstruction trial. The Journal of Thoracic and Cardiovascular Surgery, 144(4), pp.907914.

Ohye, R.G. et al., 2008. Design and rationale of a randomized trial comparing the Blalock- 
Taussig and right ventricle-pulmonary artery shunts in the Norwood procedure. The Journal of Thoracic and Cardiovascular Surgery, 136(4), pp.968-975.

Ohye, R.G. et al., 2010. Comparison of shunt types in the Norwood procedure for singleventricle lesions. New England Journal of Medicine, 362(21), pp.1980-1992.

Ohye, R.G., Schranz, D. \& d'Udekem, Y., 2016. Current Therapy for Hypoplastic Left Heart Syndrome and Related Single Ventricle Lesions. Circulation, 134(17), pp.1265-1279.

Ohye, R.G., Sleeper, L.A., et al., 2010. Comparison of shunt types in the Norwood procedure for single-ventricle lesions. New England Journal of Medicine, pp.1-13.

Pasquali, S.K. et al., 2012. Association of center volume with mortality and complications in pediatric heart surgery. Pediatrics, 129(2), pp.e370-6.

Pasquali, S.K. et al., 2013. Hospital variation in postoperative infection and outcome after congenital heart surgery. The Annals of thoracic surgery, 96(2), pp.657-663.

Pavlicek, M. et al., 2011. Right ventricular systolic function assessment: rank of echocardiographic methods vs. cardiac magnetic resonance imaging. European Journal of Echocardiography, 12(11), pp.871-880.

Pollock, E.M. et al., 1990. Early nosocomial infections in pediatric cardiovascular surgery patients. Critical care medicine, 18(4), pp.378-384.

Rácz, I. et al., 2015. Wall motion changes in myocardial infarction in relation to the time elapsed from symptoms until revascularization. The Anatolian Journal of Cardiology, 15(5), pp.363370.

Rudd, N.A. et al. 2014. Improving interstage survival after Norwood operation: outcomes from 10 years of home monitoring. The Journal of Thoracic and Cardiovascular Surgery, 148(4), pp.1540-1547.

Schidlow, D.N. et al., 2015. Site of interstage care, resource utilization, and interstage mortality: a report from the NPC-QIC registry. Pediatric Cardiology, 36(1), pp.126-131. 
Schwartz, S.M. et al., 2014. Risk factors for prolonged length of stay after the stage 2 procedure in the single-ventricle reconstruction trial. The Journal of Thoracic and Cardiovascular Surgery, 147(6), pp.1791-8-1798.e1-4.

Sergeant, P., Blackstone, E. \& Meyns, B., 1997. Can the outcome of coronary bypass grafting be predicted reliably? European journal of cardio-thoracic surgery : official journal of the European Association for Cardio-thoracic Surgery, 11(1), pp.2-9.

Sergeant, P. et al., 1997. Validation and interdependence with patient-variables of the influence of procedural variables on early and late survival after CABG. European journal of cardiothoracic surgery : official journal of the European Association for Cardio-thoracic Surgery, 12, pp.1-19.

Shahian, D.M. et al., 2015. The Society of Thoracic Surgeons Composite Measure of Individual Surgeon Performance for Adult Cardiac Surgery: A Report of The Society of Thoracic Surgeons Quality Measurement Task Force. The Annals of thoracic surgery, 100(4), pp.1315-24- discussion 1324-5.

Simsic, J.M. et al., 2004. Risk Factors for Interstage Death After the Norwood Procedure. Pediatric Cardiology, 26(4), pp.400-403.

Tabbutt, S. et al., 2012. Risk factors for hospital morbidity and mortality after the Norwood procedure: A report from the Pediatric Heart Network Single Ventricle Reconstruction trial. The Journal of Thoracic and Cardiovascular Surgery, 144(4), pp.882-895.

Taylor, L.C. et al., 2016. Risk Factors for Interstage Mortality Following the Norwood Procedure: Impact of Sociodemographic Factors. Pediatric Cardiology, 37(1), pp.68-75.

Therrien, J. et al., 2005. Optimal timing for pulmonary valve replacement in adults after tetralogy of Fallot repair. The American Journal of Cardiology, 95(6), pp.779-782.

Tweddell, J.S. et al., 2012. Intermediate-term mortality and cardiac transplantation in infants with single-ventricle lesions: Risk factors and their interaction with shunt type. The Journal of Thoracic and Cardiovascular Surgery, 144(1), pp.152-159.e2.

Welke, K.F. et al., 2009. The complex relationship between pediatric cardiac surgical case 
volumes and mortality rates in a national clinical database. The Journal of Thoracic and Cardiovascular Surgery, 137(5), pp.1133-1140.

Wilder, T.J. et al., 2016. Is a hybrid strategy a lower-risk alternative to stage 1 Norwood operation? The Journal of Thoracic and Cardiovascular Surgery.

Wilder, T.J., Caldarone, C.A., et al., 2015a. Aortic valve repair for insufficiency in older children offers unpredictable durability that may not be advantageous over a primary Ross operation†े. European journal of cardio-thoracic surgery : official journal of the European Association for Cardio-thoracic Surgery.

Wilder, T.J., McCrindle, B.W., et al., 2015b. Survival and right ventricular performance for matched children after stage-1 Norwood: Modified Blalock-Taussig shunt versus rightventricle-to-pulmonary-artery conduit. The Journal of Thoracic and Cardiovascular Surgery, 150(6), pp.1440-50-1452.e1-8- discussion 1450-2.

Wong, D.T.H. et al., 2011. Effectiveness of Serial Increases in Amino-Terminal Pro-B-Type Natriuretic Peptide Levels to Indicate the Need for Mechanical Circulatory Support in Children With Acute Decompensated Heart Failure. The American Journal of Cardiology, 107(4), pp.573-578.

Wong, J. et al., 2016. Right ventricular morphology and function following stage I palliation with a modified Blalock-Taussig shunt versus a right ventricle-to-pulmonary artery conduit. European Journal of Cardio-Thoracic Surgery. 


\title{
Copyright Acknowledgements
}

\section{For Figures 1 \& 3:}

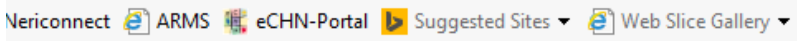 \\ Options - \\ New Exclusive Collection \\ ACCESS NOW > \\ Welcome Renew, Subscribe or Create Account $\mid$ Sign in
}

\section{The NEW ENGLAND}

\section{JOURNAL of MEDICINE}

DID YOU KNOW? A personal subscription to NEJM includes the iPad Edition, 20 FREE

Online CME exams \& more. SUBSCRIBE NOW $>>$

\begin{tabular}{|c|c|c|c|c|}
\hline номе & ARTICLES \& MULTIMEDIA * & IS SUES ${ }^{*}$ & SPECIALTIES \& TOPICS ${ }^{*}$ & FOR AUTHORS" \\
\hline
\end{tabular}

\section{Submit Your Permission Request Using Rightslink}

If you are seeking permission to copy/reproduce/republish content from NEJM and are not the author of that content, you may use the Copyright Clearance Center's Rightslink@ service. Simply visit NEJM.org and locate the article from which you seek to reuse content.

Once you have located and accessed the article you are looking for on NEJM.org

- Click on the Permission link in the article toolbox (if you are looking at the full-text), or click on the Permissions link in the middle of the page (if you are viewing the abstract or first 100 words of the article because you don't have a subscription)

- The RightsLink@ window will pop up, with information about content you have selected.

- Follow the prompts to obtain a free price quote OR

- Sign-in to your existing RightsLink® account, or create an account if you do not already have one, and follow the prompts to secure permission

Confirmation of your permission and a copy of the permission terms and conditions will be sent to you instantly via e-mail for most requests. Some requests may require Publisher review.

If you have questions about using the Rightslink® service, please contact Rightslink@ Customer Support at (877) 622 5543 (toll free) or (978) 777-9929, or e-mail customercare@copyright.com.

For other general questions about NEJM Permissions, e-mail permissions@nejm.org.

\section{Permission for Authors}

If you are the author of the article that was published in The New England Journal of Medicine ("NEJM"), please visit the NEJM Author Center.

\section{Reuse of Content within a Thesis or Dissertation}

Content (full-text or portions thereof) may be used in print and electronic versions of your dissertation or thesis without formal permission from the Massachusetts Medical Society, Publisher of the New England Journal of Medicine.

The following credit line must be printed along with the copyrighted material:

"Reproduced with permission from (scientific reference citation), Copyright Massachusetts Medical Society.

\section{Third-Party Content}

Grants of permissions apply only to copyrighted material that the MMS owns, and not to copyrighted text or illustrations for other sources.

\section{Prohibited Uses}

The New England Journal of Medicine (and its logo design) are registered trademarks of the Massachusetts Medical Society. We do not grant permission for our logo, cover or brand identity to be used in materials. Permission will not be granted for photographs depicting identifiable individuals.

\section{NEJM Information \\ Past and Present \\ Editors and Publishers \\ Editorial Policies \\ Permissions \\ Reprints \\ Upcoming Medical Conventions \\ Privacy Policy \\ Cookie Information \\ Terms of Use \\ Copyright Information \\ Frequently Asked Questions \\ Help Index \\ Contact Us \\ Content and Features \\ Overview \\ Mobile \\ What's New at NEJM.org?}

Subscriptions and Access Options

Products and Services Overview

Online Access Levels

Access from Outside the U.S.

Continuing Medical Education

Program Overview

Weekly CME Program

Review CME Program

Information For

Advertisers

Authors

Institutions

Media

Subscribers

About NEJM Group

NEJM

NEJM Knowledge+

NEJM Journal Watch

NEJM Resident 360

NEJM CareerCenter 


\section{For Figure 2:}

\section{NATURE PUBLISHING GROUP LICENSE TERMS AND CONDITIONS}

Jan 16, 2017

This Agreement between Emilie Jean-St ("You") and Nature Publishing Group ("Nature Publishing Group") consists of your license details and the terms and conditions provided by Nature Publishing Group and Copyright Clearance Center.

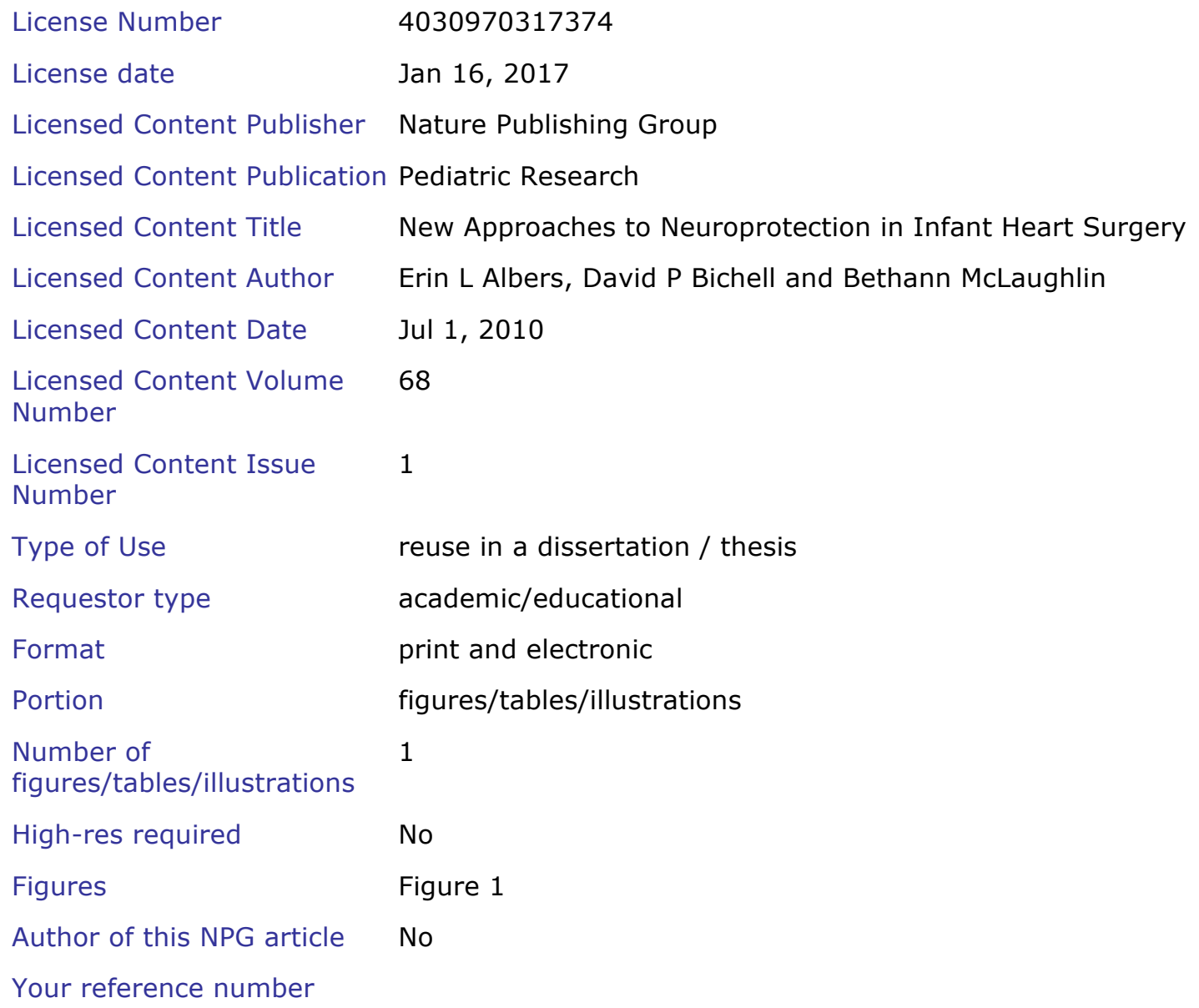


Title of your thesis /
dissertation
Expected completion date
Estimated size (number of
pages)
Requestor Location

Billing Type

Billing Address

Total

Terms and Conditions
Risk Factors for Death or Heart Transplant for Patients with Right Ventricular Dysfunction after the Horwood Procedure: A Secondary Analysis of the SIngle Ventricle Reconstruction Trial

Apr 2017

135

Emilie Jean-St-Michel

SickKids Hospital

division of cardiology

555 University avenue

Toronto, ON M5G1X8

Canada

Attn: Emilie Jean-St-Michel

Invoice

Emilie Jean-St-Michel

SickKids Hospital

division of cardiology

555 University avenue

Toronto, ON M5G1X8

Canada

Attn: Emilie Jean-St-Michel

\section{Terms and Conditions for Permissions}

Nature Publishing Group hereby grants you a non-exclusive license to reproduce this

material for this purpose, and for no other use,subject to the conditions below:

1. NPG warrants that it has, to the best of its knowledge, the rights to license reuse of this material. However, you should ensure that the material you are requesting is original to Nature Publishing Group and does not carry the copyright of another entity (as credited in the published version). If the credit line on any part of the material you have requested indicates that it was reprinted or adapted by NPG with permission from another source, then you should also seek permission from that source to reuse the material.

2. Permission granted free of charge for material in print is also usually granted for any electronic version of that work, provided that the material is incidental to the work as a whole and that the electronic version is essentially equivalent to, or substitutes for, the print version. Where print permission has been granted for a fee, separate permission must be obtained for any additional, electronic re-use (unless, as in the case of a full paper, this has already been accounted for during your initial request in the calculation of a print run).NB: In all cases, web-based use of full-text articles must be authorized separately through the 'Use on a Web Site' option when requesting permission.

3. Permission granted for a first edition does not apply to second and subsequent editions and for editions in other languages (except for signatories to the STM Permissions Guidelines, or where the first edition permission was granted for free).

4. Nature Publishing Group's permission must be acknowledged next to the figure, table or 
abstract in print. In electronic form, this acknowledgement must be visible at the same time as the figure/table/abstract, and must be hyperlinked to the journal's homepage.

5. The credit line should read:

Reprinted by permission from Macmillan Publishers Ltd: [JOURNAL NAME] (reference citation), copyright (year of publication)

For AOP papers, the credit line should read:

Reprinted by permission from Macmillan Publishers Ltd: [JOURNAL NAME], advance online publication, day month year (doi: 10.1038/sj.[JOURNAL ACRONYM].XXXXX)

\begin{abstract}
Note: For republication from the British Journal of Cancer, the following credit lines apply.

Reprinted by permission from Macmillan Publishers Ltd on behalf of Cancer Research UK: [JOURNAL NAME] (reference citation), copyright (year of publication)For AOP papers, the credit line should read:

Reprinted by permission from Macmillan Publishers Ltd on behalf of Cancer Research UK: [JOURNAL NAME], advance online publication, day month year (doi:

10.1038/sj.[JOURNAL ACRONYM].XXXXX)
\end{abstract}

6. Adaptations of single figures do not require NPG approval. However, the adaptation should be credited as follows:

Adapted by permission from Macmillan Publishers Ltd: [JOURNAL NAME] (reference citation), copyright (year of publication)

\title{
Note: For adaptation from the British Journal of Cancer, the following credit line
} applies.

Adapted by permission from Macmillan Publishers Ltd on behalf of Cancer Research UK: [JOURNAL NAME] (reference citation), copyright (year of publication)

7. Translations of 401 words up to a whole article require NPG approval. Please visit http://www.macmillanmedicalcommunications.com for more information.Translations of up to a 400 words do not require NPG approval. The translation should be credited as follows:

Translated by permission from Macmillan Publishers Ltd: [JOURNAL NAME] (reference citation), copyright (year of publication).

Note: For translation from the British Journal of Cancer, the following credit line applies.

Translated by permission from Macmillan Publishers Ltd on behalf of Cancer Research UK: [JOURNAL NAME] (reference citation), copyright (year of publication)

We are certain that all parties will benefit from this agreement and wish you the best in the use of this material. Thank you.

Special Terms:

v1.1

Questions? customercare@copyright.com or +1-855-239-3415 (toll free in the US) or 
+1-978-646-2777.

\section{For figure 5:}

\section{Order Completed}

Thank you for your order.

This Agreement between Emilie Jean-St-Michel ("You") and Wolters Kluwer Health, Inc. ("Wolters Kluwer Health, Inc.") consists of your order details and the terms and conditions provided by Wolters Kluwer Health, Inc. and Copyright Clearance Center.

\begin{tabular}{|c|c|}
\hline License number & Reference confirmation email for license number \\
\hline License date & Jan 16, 2017 \\
\hline $\begin{array}{l}\text { Licensed Content } \\
\text { Publisher }\end{array}$ & Wolters Kluwer Health, Inc. \\
\hline $\begin{array}{l}\text { Licensed Content } \\
\text { Publication }\end{array}$ & Circulation \\
\hline Licensed Content Title & Current Therapy for Hypoplastic Left Heart Syndrome and Related Single Ventricle Lesions \\
\hline Licensed Content Author & Richard G. Ohye,Dietmar Schranz,Yves D'Udekem \\
\hline Licensed Content Date & Oct 25,2016 \\
\hline Licensed Content Volume & 134 \\
\hline Licensed Content Issue & 17 \\
\hline Type of Use & Dissertation/Thesis \\
\hline Requestor type & Individual \\
\hline Portion & Figures/table/illustration \\
\hline $\begin{array}{l}\text { Number of } \\
\text { figures/tables/illustrations }\end{array}$ & 1 \\
\hline $\begin{array}{l}\text { Figures/tables/illustrations } \\
\text { used }\end{array}$ & Figure 2 \\
\hline $\begin{array}{l}\text { Author of this Wolters } \\
\text { Kluwer article }\end{array}$ & No \\
\hline
\end{tabular}


Title of your thesis / dissertation

Expected completion date Apr 2017

Estimated size(pages)

135

Requestor Location

Emilie Jean-St-Michel

SickKids Hospital

division of cardiology

555 University avenue

Toronto, ON M5G1X8

Canada

Attn: Emilie Jean-St-Michel

Billing Type

Invoice

Billing address

Emilie Jean-St-Michel

SickKids Hospital

division of cardiology

555 University avenue

Toronto, ON M5G1X8

Canada

Attn: Emilie Jean-St-Michel

Total

$0.00 \mathrm{CAD}$

\section{For figure $6 \& 7$ :}

\section{Order Completed}

Thank you for your order.

This Agreement between Emilie Jean-St-Michel ("You") and Wolters Kluwer Health, Inc. ("Wolters Kluwer Health, Inc.") consists of your order details and the terms and conditions provided by Wolters Kluwer Health, Inc. and Copyright Clearance Center.

$\begin{array}{ll}\begin{array}{l}\text { License number } \\ \text { License date }\end{array} & \text { Reference confirmation email for license number } \\ \begin{array}{l}\text { Licensed Content } \\ \text { Publisher }\end{array} & \text { Wolters Kluwer Health, Inc. } \\ \begin{array}{l}\text { Licensed Content } \\ \text { Publication }\end{array} & \text { Circulation } \\ \text { Licensed Content Title } & \begin{array}{l}\text { Does Initial Shunt Type for the Norwood Procedure Affect Echocardiographic Measures of } \\ \text { Cardiac Size and Function During Infancy?Clinical Perspective }\end{array}\end{array}$


Licensed Content Author Peter C. Frommelt,Lin T. Guey,L. LuAnn Minich,Majeed Bhat,Tim J. Bradley,Steve D.

Colan,Greg Ensing,Jessica Gorentz,Haleh Heydarian,J. Blaine John, Wyman W. Lai,Jami C. Levine, William T. Mahle,Stephen G. Miller,Richard G. Ohye,Gail D. Pearson,Girish S.

Shirali,Pierre C. Wong, Meryl S. Cohen

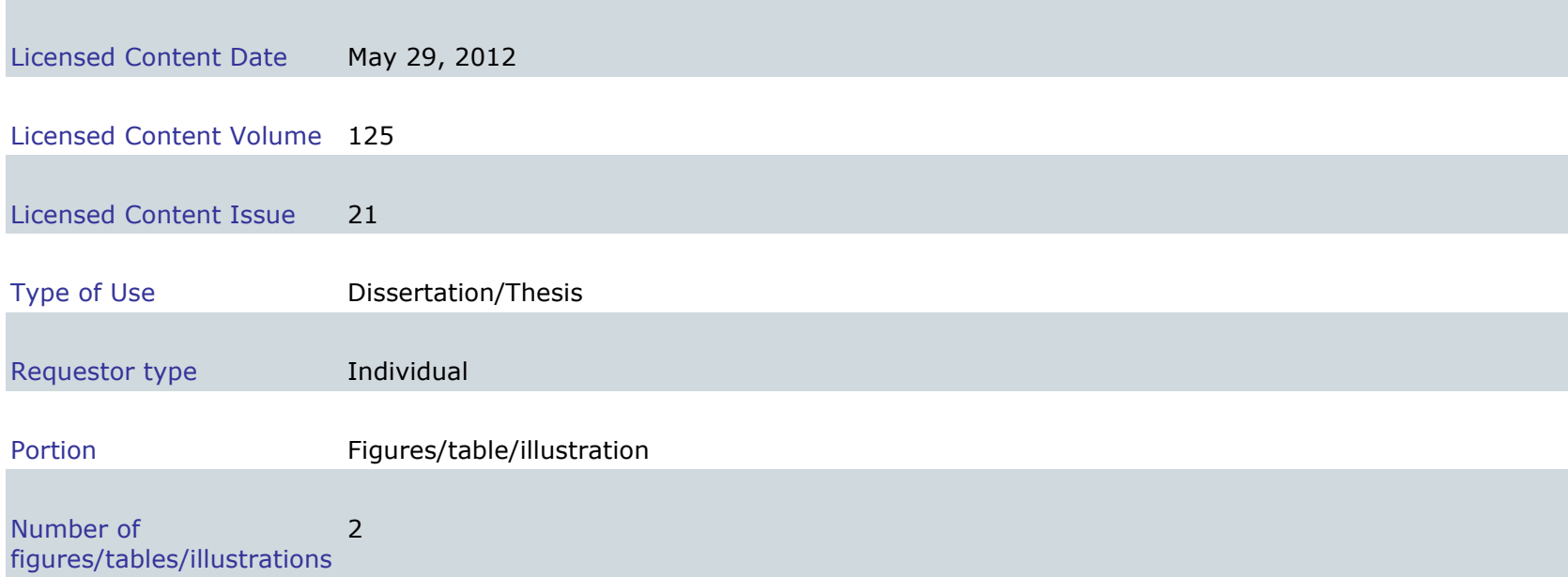

Figures/tables/illustrations Online data supplement Figure $1 \mathrm{~A}$ and $1 \mathrm{~b}$

used

Author of this Wolters No

Kluwer article

Title of your thesis /

dissertation

Risk Factors for Death or Heart Transplant for Patients with Right Ventricular Dysfunction after the Horwood Procedure: A Secondary Analysis of the SIngle Ventricle Reconstruction Trial

Expected completion date Apr 2017

Estimated size(pages) $\quad 135$

Requestor Location Emilie Jean-St-Michel

SickKids Hospital

division of cardiology

555 University avenue

Toronto, ON M5G1X8

Canada

Attn: Emilie Jean-St-Michel

Billing Type Invoice

Billing address

Emilie Jean-St-Michel

SickKids Hospital

division of cardiology

555 University avenue

Toronto, ON M5G1X8

Canada

Attn: Emilie Jean-St-Michel

Total

$0.00 \mathrm{CAD}$ 


\section{For Figure 10:}

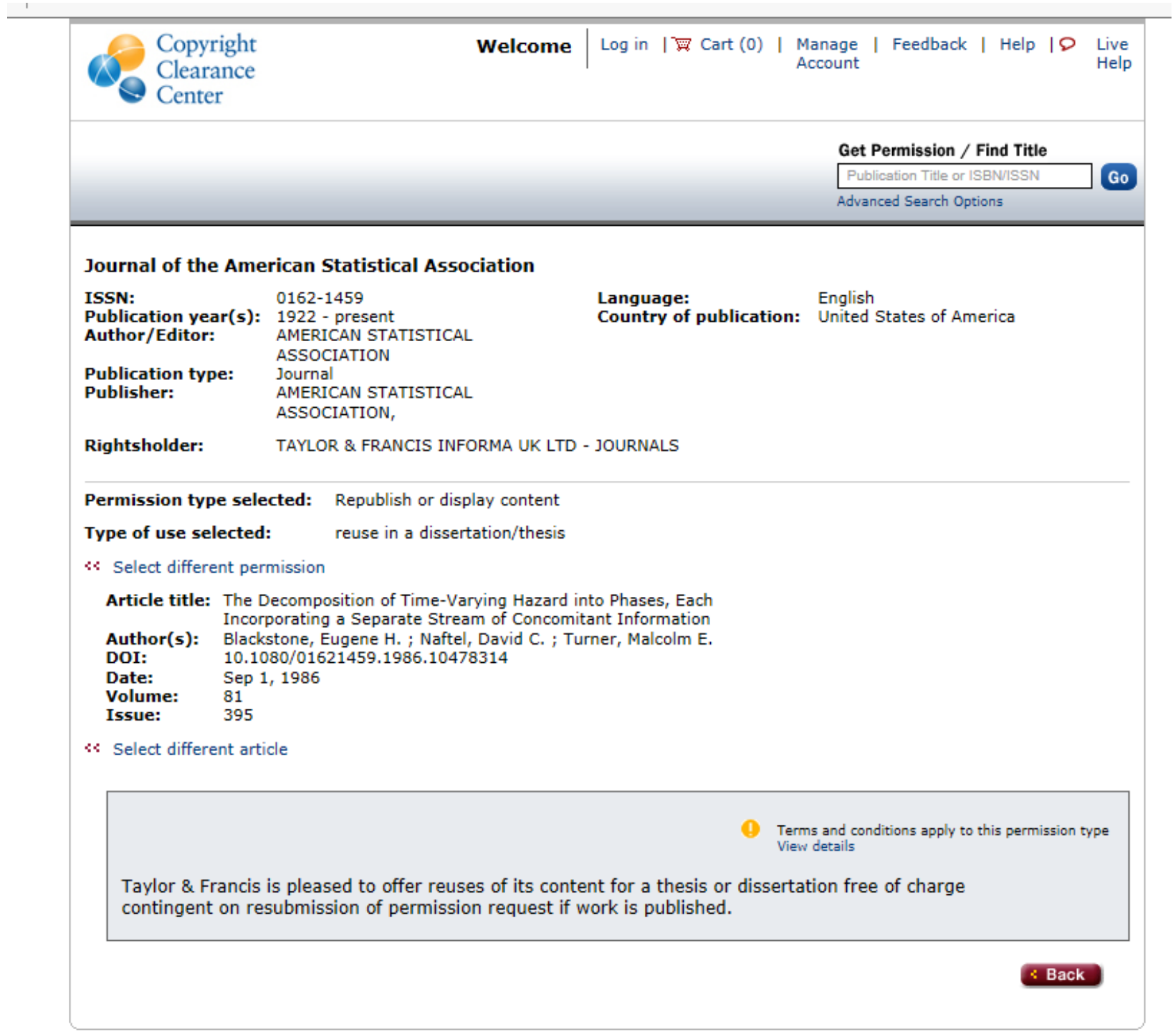

About Us | Privacy Policy | Terms \& Conditions | Pay an Invoice

Copyright 2017 Copyright Clearance Center

The original source of publication and Taylor \& Francis Ltd., are acknowledged in the caption, including a reference to the Journal's web site: http://www.informaworld.com

\section{For Figure 34:}

Thank you for your order. 
This Agreement between Emilie Jean-St-Michel ("You") and Wolters Kluwer Health, Inc. ("Wolters Kluwer Health, Inc.") consists of your order details and the terms and conditions provided by Wolters Kluwer Health, Inc. and Copyright Clearance Center.

\begin{tabular}{|c|c|}
\hline License number & Reference confirmation email for license number \\
\hline License date & Jan 16, 2017 \\
\hline $\begin{array}{l}\text { Licensed Content } \\
\text { Publisher }\end{array}$ & Wolters Kluwer Health, Inc. \\
\hline $\begin{array}{l}\text { Licensed Content } \\
\text { Publication }\end{array}$ & Circulation \\
\hline Licensed Content Title & $\begin{array}{l}\text { Use of Mathematical Modeling to Compare and Predict Hemodynamic Effects Between Hybrid } \\
\text { and Surgical Norwood Palliations for Hypoplastic Left Heart Syndrome }\end{array}$ \\
\hline Licensed Content Author & $\begin{array}{l}\text { Tain-Yen Hsia,Daria Cosentino,Chiara Corsini,Giancarlo Pennati,Gabriele Dubini,Francesco } \\
\text { Migliavacca, for the Modeling of Congenital Hearts Alliance (MOCHA) Investigators }\end{array}$ \\
\hline Licensed Content Date & Sep 13, 2011 \\
\hline Licensed Content Volume & 124 \\
\hline Licensed Content Issue & 11 suppl 1 \\
\hline Type of Use & Dissertation/Thesis \\
\hline Requestor type & Individual \\
\hline Portion & Figures/table/illustration \\
\hline $\begin{array}{l}\text { Number of } \\
\text { figures/tables/illustrations }\end{array}$ & 1 \\
\hline $\begin{array}{l}\text { Figures/tables/illustrations } \\
\text { used }\end{array}$ & Figure 4 \\
\hline $\begin{array}{l}\text { Author of this Wolters } \\
\text { Kluwer article }\end{array}$ & No \\
\hline $\begin{array}{l}\text { Title of your thesis / } \\
\text { dissertation }\end{array}$ & $\begin{array}{l}\text { Risk Factors for Death or Heart Transplant for Patients with Right Ventricular Dysfunction after } \\
\text { the Horwood Procedure: A Secondary Analysis of the SIngle Ventricle Reconstruction Trial }\end{array}$ \\
\hline Expected completion date & Apr 2017 \\
\hline Estimated size(pages) & 135 \\
\hline Requestor Location & $\begin{array}{l}\text { Emilie Jean-St-Michel } \\
\text { SickKids Hospital } \\
\text { division of cardiology } \\
555 \text { University avenue } \\
\text { Toronto, ON M5G1X8 } \\
\text { Canada } \\
\text { Attn: Emilie Jean-St-Michel }\end{array}$ \\
\hline
\end{tabular}




\begin{tabular}{ll} 
Billing Type & Credit Card \\
Credit card info & Visa ending in 6118 \\
\hline Credit card expiration & $09 / 2018$ \\
Total & 98.57 CAD
\end{tabular}

For Figure 35:

\author{
SPRINGER LICENSE \\ TERMS AND CONDITIONS
}

Jan 16, 2017

This Agreement between Emilie Jean-St ("You") and Springer ("Springer") consists of your license details and the terms and conditions provided by Springer and Copyright Clearance Center.

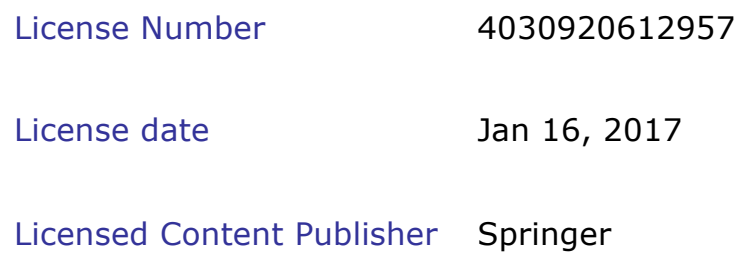


Number

Type of Use

Portion

Number of

figures/tables/illustrations

Author of this Springer

article

Order reference number

Original figure numbers

Title of your thesis / dissertation

Expected completion date

Estimated size(pages)

Requestor Location

Billing Type

Billing Address

Total
Thesis/Dissertation

Figures/tables/illustrations

1

No

Figure 1

Risk Factors for Death or Heart Transplant for Patients with Right Ventricular Dysfunction after the Horwood Procedure: A Secondary Analysis of the SIngle Ventricle Reconstruction Trial

Apr 2017

135

Emilie Jean-St-Michel

SickKids Hospital

division of cardiology

555 University avenue

Toronto, ON M5G1X8

Canada

Attn: Emilie Jean-St-Michel

Invoice

Emilie Jean-St-Michel

SickKids Hospital

division of cardiology

555 University avenue

Toronto, ON M5G1X8

Canada

Attn: Emilie Jean-St-Michel

0.0 CAD 


\section{For Figure 36:}

Terms and Conditions

This Agreement between Emilie Jean-St ("You") and Nature Publishing Group ("Nature

Publishing Group") consists of your license details and the terms and conditions provided by Nature Publishing Group and Copyright Clearance Center.

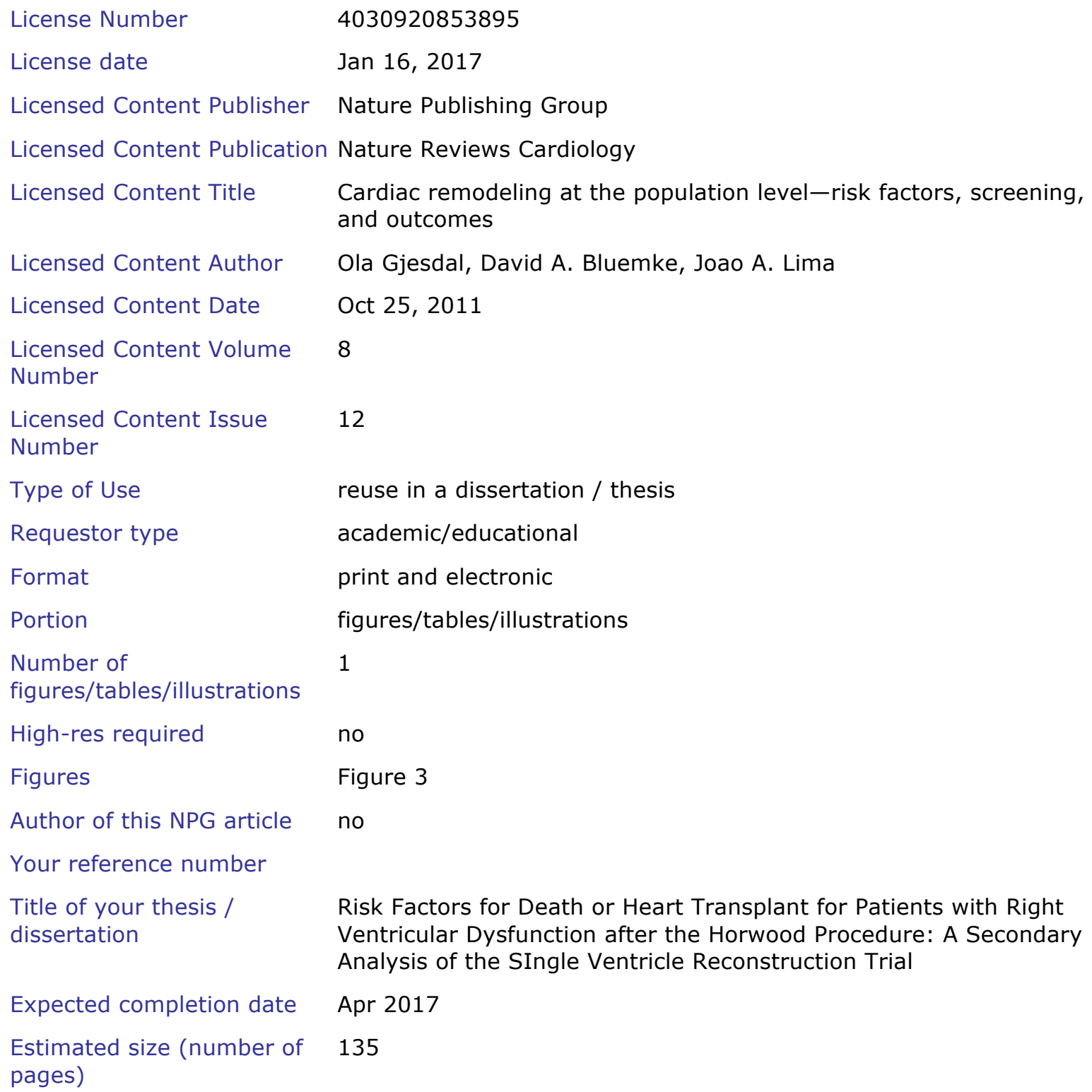

no

Risk Factors for Death or Heart Transplant for Patients with Right Ventricular Dysfunction after the Horwood Procedure: A Secondary Analysis of the SIngle Ventricle Reconstruction Trial

Apr 2017

135 


$\begin{array}{ll}\text { Requestor Location } & \text { Emilie Jean-St-Michel } \\ & \text { SickKids Hospital } \\ & \text { division of cardiology } \\ & 555 \text { University avenue } \\ & \text { Toronto, ON M5G1X8 } \\ & \text { Canada } \\ & \text { Attn: Emilie Jean-St-Michel } \\ & \text { Invoice } \\ \text { Billing Type } & \text { Emilie Jean-St-Michel } \\ \text { Billing Address } & \text { SickKids Hospital } \\ & \text { division of cardiology } \\ & 555 \text { University avenue } \\ & \text { Toronto, ON M5G1X8 } \\ & \text { Canada } \\ & \text { Attn: Emilie Jean-St-Michel } \\ & 0.00 \text { CAD }\end{array}$

Terms and Conditions

\section{Terms and Conditions for Permissions}

Nature Publishing Group hereby grants you a non-exclusive license to reproduce this material for this purpose, and for no other use,subject to the conditions below:

1. NPG warrants that it has, to the best of its knowledge, the rights to license reuse of this material. However, you should ensure that the material you are requesting is original to Nature Publishing Group and does not carry the copyright of another entity (as credited in the published version). If the credit line on any part of the material you have requested indicates that it was reprinted or adapted by NPG with permission from another source, then you should also seek permission from that source to reuse the material.

2. Permission granted free of charge for material in print is also usually granted for any electronic version of that work, provided that the material is incidental to the work as a whole and that the electronic version is essentially equivalent to, or substitutes for, the print version. Where print permission has been granted for a fee, separate permission must be obtained for any additional, electronic re-use (unless, as in the case of a full paper, this has already been accounted for during your initial request in the calculation of a print run).NB: In all cases, web-based use of full-text articles must be authorized separately through the 'Use on a Web Site' option when requesting permission.

3. Permission granted for a first edition does not apply to second and subsequent editions and for editions in other languages (except for signatories to the STM Permissions Guidelines, or where the first edition permission was granted for free).

4. Nature Publishing Group's permission must be acknowledged next to the figure, table or abstract in print. In electronic form, this acknowledgement must be visible at the same time as the figure/table/abstract, and must be hyperlinked to the journal's homepage.

5. The credit line should read:

Reprinted by permission from Macmillan Publishers Ltd: [JOURNAL NAME] (reference 
citation), copyright (year of publication)

For AOP papers, the credit line should read:

Reprinted by permission from Macmillan Publishers Ltd: [JOURNAL NAME], advance

online publication, day month year (doi: 10.1038/sj.[JOURNAL ACRONYM].XXXXX)

\section{Note: For republication from the British Journal of Cancer, the following credit}

lines apply.

Reprinted by permission from Macmillan Publishers Ltd on behalf of Cancer Research UK: [JOURNAL NAME] (reference citation), copyright (year of publication)For AOP papers, the credit line should read:

Reprinted by permission from Macmillan Publishers Ltd on behalf of Cancer Research UK: [JOURNAL NAME], advance online publication, day month year (doi:

10.1038/sj.[JOURNAL ACRONYM].XXXXX)

6. Adaptations of single figures do not require NPG approval. However, the adaptation should be credited as follows:

Adapted by permission from Macmillan Publishers Ltd: [JOURNAL NAME] (reference citation), copyright (year of publication)

Note: For adaptation from the British Journal of Cancer, the following credit line applies.

Adapted by permission from Macmillan Publishers Ltd on behalf of Cancer Research UK: [JOURNAL NAME] (reference citation), copyright (year of publication)

7. Translations of 401 words up to a whole article require NPG approval. Please visit http://www.macmillanmedicalcommunications.com for more information.Translations of up to a 400 words do not require NPG approval. The translation should be credited as follows:

Translated by permission from Macmillan Publishers Ltd: [JOURNAL NAME] (reference citation), copyright (year of publication).

\section{Note: For translation from the British Journal of Cancer, the following credit line applies. \\ Translated by permission from Macmillan Publishers Ltd on behalf of Cancer Research \\ UK: [JOURNAL NAME] (reference citation), copyright (year of publication)}

We are certain that all parties will benefit from this agreement and wish you the best in the use of this material. Thank you.

Special Terms:

v1.1 